\title{
Experimental heart failure in rats : hemodynamic studies on pathophysiology and therapy
}

Citation for published version (APA):

Schoemaker, R. G. (1989). Experimental heart failure in rats : hemodynamic studies on pathophysiology and therapy. [Doctoral Thesis, Maastricht University]. Datawyse. https://doi.org/10.26481/dis.19891207rs

Document status and date:

Published: 01/01/1989

DOI:

10.26481/dis.19891207rs

Document Version:

Publisher's PDF, also known as Version of record

\section{Please check the document version of this publication:}

- A submitted manuscript is the version of the article upon submission and before peer-review. There can be important differences between the submitted version and the official published version of record.

People interested in the research are advised to contact the author for the final version of the publication, or visit the DOI to the publisher's website.

- The final author version and the galley proof are versions of the publication after peer review.

- The final published version features the final layout of the paper including the volume, issue and page numbers.

Link to publication

\footnotetext{
General rights rights.

- You may freely distribute the URL identifying the publication in the public portal. please follow below link for the End User Agreement:

www.umlib.nl/taverne-license

Take down policy

If you believe that this document breaches copyright please contact us at:

repository@maastrichtuniversity.nl

providing details and we will investigate your claim.
}

Copyright and moral rights for the publications made accessible in the public portal are retained by the authors and/or other copyright owners and it is a condition of accessing publications that users recognise and abide by the legal requirements associated with these

- Users may download and print one copy of any publication from the public portal for the purpose of private study or research.

- You may not further distribute the material or use it for any profit-making activity or commercial gain

If the publication is distributed under the terms of Article $25 \mathrm{fa}$ of the Dutch Copyright Act, indicated by the "Taverne" license above, 


\section{EXPERIMENTAL HEART FAILURE IN RATS}

hemodynamic studies on pathophysiology and therapy 



\title{
EXPERIMENTAL HEART FAILURE IN RATS
}

\section{hemodynamic studies on pathophysiology and therapy}

\section{PROEFSCHRIFT}

\author{
ter verkrijging van de graad van doctor \\ aan de Rijksuniversiteit Limburg te Maastricht, \\ op gezag van de Rector Magnificus, prof. dr. F.I.M. Bonke, \\ volgens het besluit van het College wan Dekanen, \\ in het openbaar te verdedigen \\ op donderdag, 7 december 1989 om 14.00 uur
}

door

Regina Geertruida Schoemaker geboren te Meppel in 1958 
Promotor

Co-promotor

Beoordelingskommissie
Prof. dr. H.A.J. Struyker Boudier

Dr. J.F.M. Smits

Prof. dr. F.T. Bosman

Dr. H. Drexler

Prof. dr. R.S. Reneman

Prof. dr. H.H.J. Wellens

Prof. dr. P.A. van Zwieten

\section{CIP-GEGEVENS KONINKLIJKE BIBLIOTHEEK, DEN HAAG}

\section{Schoemaker, Regina Geertruida}

Experimental heart failure in rats: hemodynamic studies on pathophysiology and therapy / Regina Geertruidat

Schocmaker. - Maastricht: Datawyse. - III.

Thesis Matstricht. - With rof. - With summery in dutch.

ISBN 90-5291-013\%8.

SISO 605.12 UDC 616.12(043.3)

Subject heading: heart failure

Uitgave: Datawyse * Maastricht

Druk: Krips Repro Meppel

De uitgave van dit proefschrift werd mede mogelijk gemaakt door de financiele steun van de firma's Squibb en Sterling Winthrop in Nederland. 
voor Hans

aan mijn ouders 


\section{LIST OF ABBREVIATIONS}

ACE Angiotensin I converting enzyme

BW Body weight

Cl Cardiac index

CO Cardiac output

CVP Central venous pressure

$\mathrm{dF} / \mathrm{dt} \quad$ Maximal flow acceleration

HR Heart rate

HW Heart weight

LVEC Left ventricular endocardial circumference

LVEDP Left ventricular end-diastolic pressure

LVET Left ventricular ejection time

MAP Mean arterial pressure

PEP Pre-ejection period

PF Peak flow

QS Duration QS interval

resp. Respiration rate

SV Stroke volume

SW Stroke work

TPR Total peripheral resistance 


\section{TABLE OF CONTENTS}

1. Introduction

1.1 General

1.2 Therapy

1.3 Pathophysiology $\quad 4$

1.3.1 Peripheral compensation 4

1.3.2 Cardiac compensation 6

1.4 Animal models

1.5 The present thesis 11

2. Materials and Methods $\quad 13$

2.1 Animals $\quad 13$

2.2 Coronary artery ligation $\quad 13$

2.2.1 Chromic ligation

2.2.2 Acute ligation $\quad 14$

2.3 Infarct size measurements 14

2.4 Preparations for hemodynamic measurements $\quad 15$

$\begin{array}{lc}2.4 .1 \text { Cardiac output } & 15\end{array}$

2.4.2 Arterial blood pressure 16

2.4 .3 Central venous pressure $\quad 17$

2.4 .4 Intravemous infusion $\quad 17$

2.4.5 Electrocardiogram $\quad 17$

$\begin{array}{lr}2.5 \text { Hemodynamic measurements } & 19\end{array}$

2.5.1 Method for quantifying heart failure

2.5.2 Evaluation of method for quantifying heart failure $\quad 21$

2.6 Ejection fraction measurements $\quad 23$

$\begin{array}{lr}2.7 \text { Statistics } & 23 \\ 2.8 & 23\end{array}$

$\begin{array}{ll}2.8 \text { Drugs used in this thesis } & 23\end{array}$

3. The aging spontaneously hypertensive rat as a model for heart fallure 25

$\begin{array}{lr}3.1 \text { Introduction } & 25\end{array}$

3.2 Experimental protocols $\quad 26$

3.2.1 Hemodynamic mexsurements $\quad 26$

3.2 .2 Ejection fraction measurements $\quad 26$

$\begin{array}{lr}3.3 \text { Resnlts } & 27\end{array}$

3.4 Discussion $\quad 32$

4. The coronary artery ligated rat as a model for heart failure 37

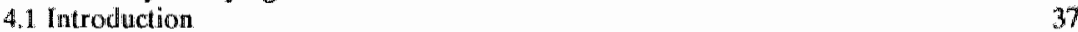

4.2 Experimental protocols $\quad 37$

4.2.1 Acute effects of coronary artery ligation $\quad 38$

4.2 .2 Long-term effects of coronary artery ligation $\quad 39$

$\begin{array}{ll}43 \text { Results. } & 39\end{array}$

4.3.1 Coronary artery ligation $\quad 39$

4.3 .2 Effects of coronary artery ligation 42

$\begin{array}{lr}4.4 \text { Discussion } & 47\end{array}$

$\begin{array}{ll}4.4 .1 \text { Acute elfects of infarction } & 47\end{array}$

4.4.2 Long-term effects of infarction 48

$\begin{array}{ll}\text { 5. Milrinone in heart failure } & 51\end{array}$

$\begin{array}{ll}5.11 \text { Introduction } & 51\end{array}$

$\begin{array}{ll}5.2 . & \text { Experimental protocols }\end{array}$

5.2 .1 Acute effects in normal bealthy rats 52

5.2 .2 Acute effects in chronically infarcted rais $\$ 2$

5.2.3 Two weeks therapy in chronically infarcted rats $\quad 53$

$\begin{array}{ll}5.3 \text { Results } & 53\end{array}$

5.3 .1 Acute effects in normal healty rats

5.3.2 Acute effects in chronically infarcted rats

5.3.3 Effects of two weeks therapy in chronically infarcted ratis. 56 
5.4.1 Acute effects of milrinone in normal healthy rats 60

5.4.2 Milninone in rats with heart failure 60

6. Doblutaminie in heart failure

6.1 Introduction 63

6.2 Experimental protocols 64

6.2.1. Acute effects in normal healthy rats 64

6.2.2 Two weeks therapy in tats with heart failure followed by acute administration 64

63 Results

6.3.1 Acute effects in normal healthy rats

6.3.2 Effects of 2 weeks therapy in infarcted rats 65

6.3 .3 Effects of acute dobutamine after 2 weeks of therapy 70

6.4 Discussion 72

7. Angiotensin converting enzyme inhibition 77

A. Captoprill in heart fallure 77

$\begin{array}{ll}7.1 \text { Introdluction } & 77\end{array}$

7.2 Experimental protocols 78

7.2.1 Acute effects of captopril in chronically infarcted rats 78

7.2 .2 Effects of 2 weeks captopril after the healing period 78

7.2.3 Effects of captopril therapy during the healing period 79

7.3 Resuits $\quad 79$

7.3.1 Acute effects of captopril in chronically infarcted rats 79

7.3.2 Effects of 2 weeks captopril after the healing period 79

7.3.3 Effects of captopril therapy during the healing period

7.4 Discussion

B. Benazepril in heart failure

7.5 Introduction 91

7.6 Experimental protocols

7.6.1 Dose finding study $\quad 92$

7.6.2 Hemodynamic study

7.7 Results

7.7.1 Dose finding study 93

7.7.2 Hemodynamic study

$\begin{array}{ll}7.8 \text { Discussion } & 97\end{array}$

8. Concluding remarks and outlook 99

8.1 General 99

8.2 Models, pathophysiology and therapy 99

8.2.1 Early captopril 100

$\begin{array}{ll}8.2 .2 \text { Later captopril } & 100\end{array}$

8.2 .3 Dobutamine 101

8.2.4 Milrinone 102

8.3 Future oullook 102

Refterences 103

$\begin{array}{ll}\text { Summary } & 113\end{array}$

Samienvatting $\quad \mathbf{1 1 5}$

$\begin{array}{ll}\text { Curriculum vitae and list of publications } & 117\end{array}$

$\begin{array}{ll}\text { Ter afsluiting } & 119\end{array}$ 


\section{INTRODUCTION}

"The use of the Foxglove is getting abroad, and it is better the world should derive some instruction, however imperfect, from my experience, than that the lives of man should be hazarded by its untegardod exposition, or that a medicine of so mach efficacy should be condemned and rejected as dangerous and unmanageable".

William Withering, An account of the Foxglowe, 1785 .

\subsection{General}

Now, 200 years later, cardiac glycosides still are the mainstay of treatment in congestive heart failure despite the concern of their small therapeutic/toxic dose range. Nevertheless, considerable advances have been made in the treatment of congestive heart failure. Of special importance was the introduction of diuretics in the 1940 's and of vasodilators in the 1970's. However, although many new therapeutic agents have been developed which actually reduce symptoms, overall mortality still has not been significantly reduced.

Despite the prevalence of the problem in medical practice, little was known about the epidemiology of congestive heart failure prior to the Framingham study in the early 1970's (McKee et al, 1971). The natural history of congestive heart failure was studied over a 16-year period in more than 5000 persons free of overt manifestations of the disease at entry. It appeared that congestive heart failure is not a disease per se but a common clinical manifestation of many different heart diseases. In fact, all forms of heart diseases may lead to ventricular dysfunction and cause the syndrome of heart failure (table 1.1).

Because prevalence of heart failure increases with age, and because patients with cardiac diseases live longer due to advances in medical and surgical management, the number of patients who develop heart failure is expected to increase. Now it is estimated that more than 5 million people in the United States suffer from heart failure and that more than 500,000 new cases are diagnosed every year (Cohn, 1988). Once the syndrome is manifest, the mortality rate is very high, approximately $50 \%$ within the first year. Until now, therapy has not been able to improve prognosis significantly. 
Table 1.1. Cardiovascular diseases which can lead to congestive heart failure (Remme, 1986)

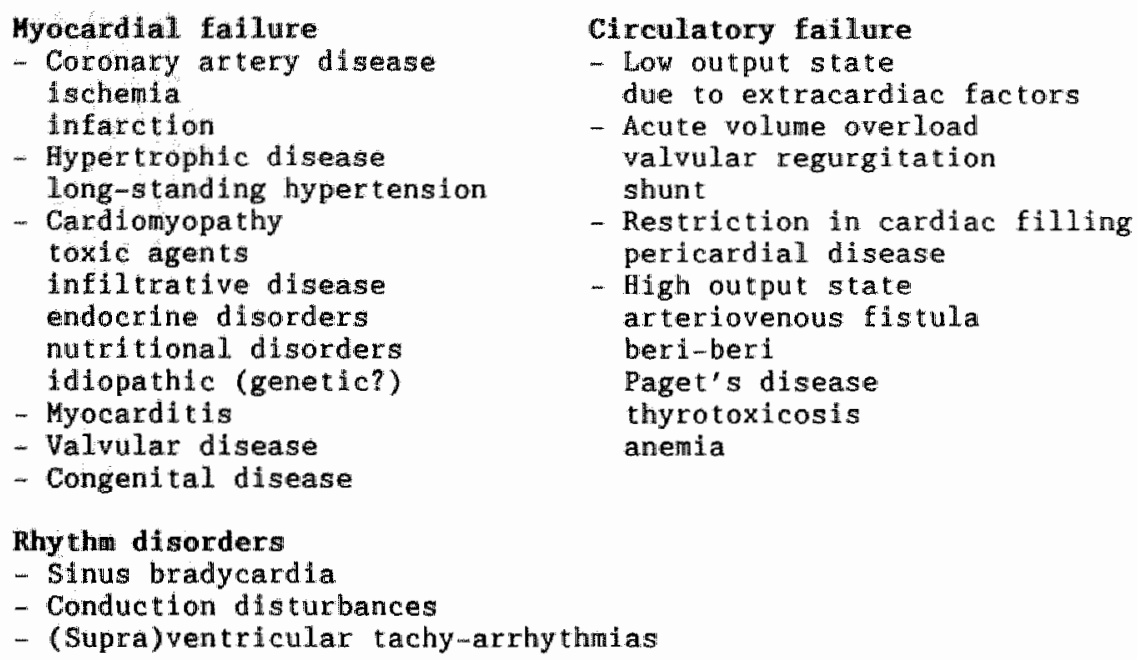

Myocardial fallure

- Coronaxy artery disease ischemia infarction

- Hypertrophic disease long-standing hypertension

- Cardiomyopathy toxic agents infiltrative disease endocrine disorders nutritional disorders idiopathle (genetic?)

- Myocarditis

- Valvular disease

- Congenital disease

Rhy thm disorders

- Sinus bradycardia

- Conduction disturbances

- (Supra)ventricular tachy-arrhythmias

\section{Circulatory faillure}

- Low output state due to extracardiac factors

- Acute volume overload valvular regurgitation shunt

- Restriction in cardiac filling pericardial disease

- High output state arteriovenous fistula beri-beri Paget's disease thyrotoxicosis anemia

\section{Table 1.2 The three main pharmacological approaches to heart failure}

1. Positive inotropic agents

- digitalis glycosides

- sympathomimetic drugs

- phosphodiesterase inhibitors

2. Diuretics

3. Vasodilating agents

- nitrates

- ACE inhibitors

- calcilum channel blockers

- alpha-adrenergic antagonists

- centrally acting vasodilators

\subsection{Therapy}

For the treatment of heart failure, physicians today can make a choice from a variety of therapeutic agents, each with its own specific properties and limitations. Three major groups of agents can be distinguished and are briefly described (table 1.2). 
Positive inotropic agents can grossly be divided into cardiac glycosides, like digitalis; sympathicomimetics, such as isoprenaline, dobutamine and xamoterol, and phosphodiesterase inhibitors, with and without Ca-sensitizing activity, sulmazole and milrinone, respectively. The major action of these drugs is to increase myocardial contractility by increasing intracellular levels of $\mathrm{Ca}^{2+}$ or by increasing the sensitivity of the contractile elements to $\mathrm{Ca}^{2+}$. Effects of $\mathrm{Ca}^{2+}$ mobilization are extensively reviewed by Reiter (1988). Most agents are reported to improve hemodynamic state and exercise tolerance (LeJemtel et al, 1984; Colucci et al, 1986a, b, Farah, 1986; Braunwald, 1986; Wetzel et al, 1988). However, as a consequence of the increased contractility, myocardial oxygen consumption will increase. In hearts in which energy supply has already been diminished, in the end, this may increase cell damage (Katz, 1986). The reduced inotropic response of failing hearts to a variety of positive inotropic agents (Brown et al, 1986) may then suggest a natural adaptive process. In addition, most of the positive inotropic agents have arrhythmogenic activity (Parmley et al, 1986; Colucci et al, 1986a,b; Katz, 1986). Moreover, positive inotropic therapy is not associated with increased survival (Packer et al, 1987a; Cohn, 1989; DiBianco et al, 1989). Thus, although positive inotropes can acutely improve the condition of the patient, effects on myocardial energy demand, in addition to arrhythmogenic activity of most agents, may limit the use of these agents in chronic therapy.

Diuretic agents are primarily directed towards relief of the symptoms of systemic and pulmonary congestion. Mild heart failure can be satisfactorily treated with a diuretic alone, like hydrochlorothiazide. Most patients, however, need additional therapy (Ruggie, 1986). The loop diuretics, like furosemide, are potent short-acting agents and are of special benefit in the treatment of acute pulmonary oedema. Diuretics are self-limiting: when circulating volume decreases, cardiac filling decreases and consequently cardiac output, renal flow and glomerular filtration rate decrease (Nelson et al, 1984). Furthermore, diuretics stimulate the renin-angiotensin-aldosterone system leading to increased vascular resistance (Remme, 1986; Bayliss et al, 1987). Electrolyte disturbances, such as potassium loss, constitute an adverse side-effect, especially when digitalis is given simultaneously (Ruggie, 1986). Thus, although diuretics relieve symptoms of congestion and improve the clinical state, their value for chronic therapy may be limited.

Vasodilators are used to unload the heart without direct action on ventricular contractility. Different groups of agents can be distinguished based on mechanism of action: direct vasodilators such as sodium nitroprusside, nitrates, hydralazine and minoxidil; alpha-1 adrenoceptor blockers, such as prazosin; centrally acting sympatholytics, such as alpha-methyldopa; Ca-channel blockers, like nifedipine, and angiotensin converting enzyme inhibitors, such as captopril and enalapril (Levine, 1985). Hydralazine and nifedipine have predominantly arterial vasodilating effects, while nitroglycerin and isosorbide dinitrate have predominantly venodilating effects. Captopril and enalapril as well as prazosin have balanced venous and arterial effects (reviewed by Levine, 1985; Packer, 1986; Sharpe, 1989). Despite these differences, all have been shown to favorably alter the hemodynamic pattern in patients with heart failure. Unfortunately, this short-term hemodynamic response is not a reliable guide to favorable long-term effects because of the development of tolerance and counterregulation by neurohumoral reflex mechanisms (Levine, 1985; Packer, 1986; Chatterjee et al, 1988). Selective arterial vasodilatation may further increase preload as at result of reduced renal function due to a lower blood pressure. Selective venodilation may reduce the cardiac filling, that is needed to preserve cardiac output, as occurs 
after myocardial infarction (Ruggie, 1986; Remme, 1986). Simultaneous reduction of preload and afterload was suggested to be desirable (Freis, 1984). In fact, the only type of long-term therapy which has actually been reported to increase survival, enalaprill (Consensus Trial, 1987) and combined therapy with hydralazine and isosorbide dinitrate (Cohn et al, 1986), supports the suggestion that a balanced venous and arterial vasodilation is beneficial. Pfeffer et al (1988) suggested that chronic captopril therapy can attenuate ventricular dilatation after myocardial infarction, while Sharpe et al (1988) showed a reduced left ventricular end-diastolic volume. These observations suggest that besides relief of symptoms, progression of the disease can be influenced, which may improve prognosis. Nevertheless, vasodilating therapy, so far, is the only therapy which can clinically improve the patient and also prolong life expectancy.

Despite the variety of therapeutic agents and the latter promising data, once congestive heart failure is diagnosed, prognosis still remains very poor. Therefore, it has been suggested to start therapy even before symptoms become overt (Gorlin, 1987; Sharpe, 1989). In the early stages, changes might be reversible, whereas at later stages especially when cell death has occurred, the only effect that can be achieved with therapy may be symptomatic relief. However, the early signs of heart failure, breathlessness and fatigue, are rather non-specific symptoms. Recognition of clinical presentation usually depends upon evidence of fluid retention. These peripheral symptoms may be the symptoms of end-stage failure. Therapy usually is too much focussed on these symptoms. More attention should be paid to the pathophysiological conditions leading to failure, implying a better understanding of the pathophysiology.

\subsection{Pathophysiology}

Congestive heart failure is classically defined as the pathophysiological state in which the cardiac output is insufficient to meet the metabolic needs of the body (Ruggie, 1986). Actually, in the case of a failing heart, supply exceeds the pump capacity of the heart and leads to congestion. The most commonly encountered variety of heart failure is a complex clinical syndrome resulting form both cardiac dysfunction and peripheral compensatory mechanisms in response to a decrease in cardiac output (Ruggie, 1986). Therefore, in the following, the term "heart failure" will refer to this type of failure and thus exclude the phenomenon of high output failure.

When the heart as a pump begins to fail, it remains able to meet the demands of the body because the circulatory system compensates by producing vasoconstriction to maintain blood pressure and volume expansion to maintain cardiac output (Katz, 1987). Also in the myocardium itself changes occur to compensate the impaired function (Meerson, 1983). Fig. 1.1 summarizes the major mechanisms.

\subsubsection{Peripheral compensation}

In acute heart failure, the reduction of cardiac output leads to increased sympathetic stimulation of the heart and the peripheral vasculature leading to increased contractility and arteriolar vasoconstriction (Remme, 1986). If cardiac output remains 


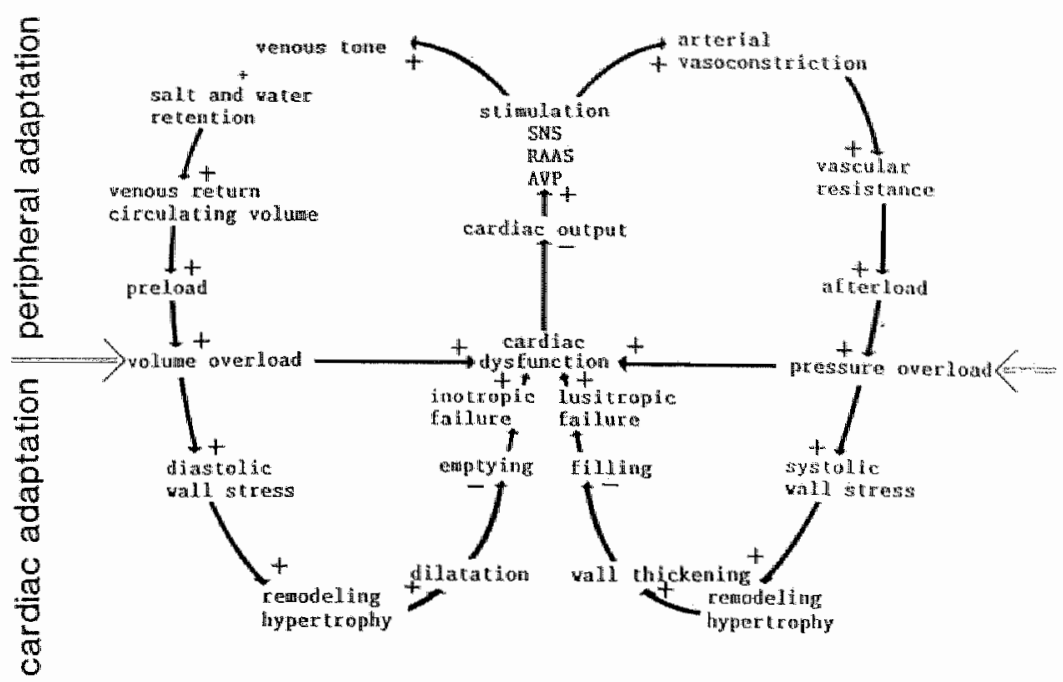

Fig. 1.1 Peripheral and cardiac mechanisms involved in decompensation. SNS: sympathetic nervous system; RALAS: renin-angiotensin-aldosterone system; AVP: aPginine-vasopressin system

depressed, endocrine systems besides the sympathetic nervous system, become activated as well.

Activation of the renin-angiotensin-aldosterone system results in an increased level of angiotensin II which has several effects in heart failure. Firstly, angiotensin II is a potent vasoconstrictor and, thus, directly contributes to the increase in systemic vascular resistance. Secondly, it facilitates noradrenalline release during sympathetic stimulation. Thirdly, it leads to an increased release of aldosterone which further promotes retention of salt and water (Francis, 1985; Parmley, 1985; Laragh, 1986). Overactivity of the renin-angiotensin-aldosterone system is found in heart failure (Laragh, 1986), although a response to routine diuretic treatment could have contributed to an increased activity as well (Bayliss et al, 1987). Activation of arginine vasopressin further contributes to vasoconstriction and water reabsorption (Remme, 1986).

The functional counterpart of the above systems is the atrial natriuretic peptide (ANP) (Genest, 1986; Morris et al, 1987). ANP levels appear to be increased even in the early stages of heart failure. In non-treated patients, the increase in ANP level is proportionally larger than the increases in plasma renin, aldosterone and antidiuretic hormone (Fyhrquist et al, 1988). ANP infusion in patients with heart failure reduces preload and afterload to both ventricles, without effects on heart rate, cardiac index or myocardial oxygen consumption (Herrmann et al, 1988). When pump failure becomes more severe, the counterbalancing effects of ANP, diuresis, natriuresis and vasodilatation, may be overwhelmed by vasoconstriction and fluid retention (Fyhrquist et al, 1988).

Moreover, in congestive heart failure, the attempts to maintain perfusion 
pressure may have adverse secondary effects. The deleterious consequences of increased afterload on the impaired ventricular function, and increased preload on an already congested circulation (Francis et al, 1984), can eventually result in a vicious circle (cf. fig. 1.1). These peripheral responses rather than the cardiac dysfunction itself are responsible for the signs and symptoms of congestive heart failure.

\subsubsection{Cardiac compensation}

The pathophysiological conditions leading to congestive heart failure result from pressure or volume overload, loss of viable myocardium or a generalized decrease in myocardial contractility (fig. 1.2). Pressure overload is seen in hypertension or aortic stenosis, while volume overload includes aortic and mitral regurgitation as well as left to right shunts. A generalized decrease in myocardial contractility can be seen in dilated cardiomyopathy. The most common cause of heart failure is coronary artery disease in which myocardial ischemia or infarction results in the loss of myocardium and its replacement with non-contractile scar tissue (Ruggie, 1986).

The type of hemodynamic stress to which the ventricle is subjected appears to determine the nature of the compensation, since the cardiac response is directed to normalize wall stress (Meerson, 1983). In accordance with Laplace's law - T=P.r/2h -, wall tension $T$ is equal to the product of the pressure in the ventricle (P), multiplied by the radius of curvature of the ventricle ( $r$ ) and divided by twice the thickness of the ventricle wall (2h) (Meerson, 1983). Effects on the shape of the heart resulting from these responses are illustrated in fig. 1.2 (Gorlin, 1987).

In patients with pressure overload, the increase in systolic wall stress leads to replication of sarcomeres in parallel to the existing ones, resulting in increased wall thickness (Meerson, 1983; Ruggie, 1986). This, however, may lead to a preserved systolic function at the expense of diastolic function, i.e. lusitropic failure (Katz, 1987). In patients with volume overload the initial increase in wall stress occurs in diastole. This leads to replication of sarcomeres in series, resulting in fiber elongation and chamber enlargement (Ruggie, 1986). Without concomitant wal thickening, this response may preserve relaxation and filling but results in inotropic or ejection failure (Katz, 1987). However, the attendant increase in chamber radius at end systole then results in increased systolic wall stress (fig. 1.1), leading to concomitant wall thickening (Ruggie, 1986). Structural dilatation as indicated by increased cavity to wall ratio (Vogt et $\mathrm{al}_{*}$ 1987a) implies higher wall stress and therefore higher energy demand. In this regard, the situation of patients with recurrent infarctions and chronic heart failure resembles chronic volume overload as does that of patients with dilated cardiomyopathy.

In compensated patients, changes in ventricular shape and wall thickness allow the heart to adapt to the increased load. Although compensated, hearts are not normal and metabolic and mechanical alterations have been identified (Katz, 1989). These alterations include increased maximal oxygen diffusion distance due to increased myocyte size, increased interstitial tissue volume and decreased capillary density (Meerson, 1983). This results in a decreased oxygen supply to the myocytes. Decreased mitochondria to myofibril ratio (Anversa et al, 1986) indicates imbalance between energy generating and energy demanding elements. Furthermore, impaired $\mathrm{Ca}^{2+}$ re-uptake in sarcoplasmatic reticulum (Ito et al, 1974; Katz, 1989), results in impaired contraction and, possibly even more important, impaired relaxation (Meer 


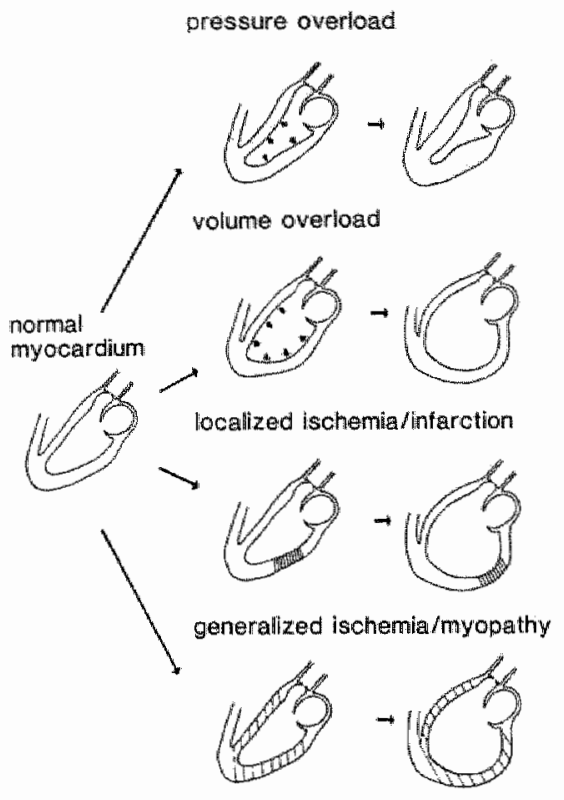

Fig. 1.2 Effects of different types of stress and consequent remodeling on the shape of the heart

son, 1983; Katz, 1989). Impaired relaxation facilitates the effects of diminished oxygen supply by reducing coronary blood flow. Furthermore, a shift in cardiac myosin isoenzymes towards a slower contracting type of fibers has been reported which is associated with lower ATP-ase activity and decreased $\mathrm{Ca}^{2+}$ sensitivity (Gorza et al, 1984; Morano et al, 1988; Bouvagnet et al, 1989). Effects are related to increased cardiac load (Hoffmann et al, 1987; Schaub et al, 1987).

The complexity of peripheral and cardiac compensatory mechanisms makes it difficult to determine when adaptation develops into de-adaptation. Peripheral compensatory mechanisms could facilitate the development of failure by causing overload to the heart. Symptomatic relief after therapy indicates that these mechanisms can be affected, which, however, does not necessarily imply improved survival. Also cardiac compensatory mechanisms could facilitate development of failure. Remodeling of the heart may lead to structural dilatation and chronically increased wall stress. Intervention in the course of cardiac changes could then delay or prevent development of failure. It is known from a regression of cardiac hypertrophy due to antihypertensive therapy (Sen, 1982) that drugs can affect cardiac remodeling.

In two recent studies in patients with myocardial infarction, who were not in overt heart failure, captopril therapy reduces left ventricular end-diastolic volume compared to placebo (Sharpe et al, 1988) and is suggested to attenuate left ventricular dilatation (Pfeffer et al, 1988). Studies suggest that interaction with cardiac remodeling provides a new opportunity for therapy. In order to investigate this opportunity, drugs should be studied at comparable degree of improvement of peripheral symptoms but presumably different effects on cardiac remodeling. The 
hypothesis is that effective drug therapy of heart failure should interact with peripheral processes to reduce symptoms, and intervene with cardiac remodeling to inhibit progression of the cardiac disease. Obviously, verification of this hypothesis is very difficult to study in patients. Therefore, research implies the availability of a suitable animal model for heart failure to study pathophysiology and treatment.

\subsection{Animal models}

Over the years, several animal models for heart failure have been developed (reviews Czarnecki, 1984; Smith et al, 1985; Curtis et al, 1987) mostly based upon conditions that lead to heart failure in man (table 1.3).

It is possible to describe a set of criteria which can be used to assess the animal models for heart failure. An ideal model would (1) completely mimic one or more of the various clinical conditions; (2) respond to drugs in a manner that corresponds with the clinical response; (3) have sufficient precision and accuracy to function as a bioassay; (4) permit a variety of responses to be measured, and (5) be undemanding in terms of costs, time and expertise (Curtis et al, 1987).

In vitro approaches with isolated papillary muscles or isolated Langendorff hearts may be helpful in defining the state of the myocardium. However, the clinical picture arises from the dynamic interactions of hemodynamic, nervous, endocrine and structural mechanisms. These can only be studied in intact conscious animals.

For studies on pathophysiology and therapy, the first two criteria are the most important ones because they are a measure for clinical significance of the model. The most obvious clinical condition to mimic is cardiac dysfunction since this is the central problem in heart failure (fig. 1.1). With regard to criterion 5 , a model with small animals is preferred. With the mentioned criteria and restrictions in mind several models are evaluated in the following paragraphs.

Cardiomyopathies may be induced with a number of chemical agents including barbiturates, adriamycin (doxorubicin), streptozotocin, furazolidone, cobalt, alcohol and catecholamines (for a review, see Czarnecki, 1984; Smith et al, 1985). Most research has been focussed on cellular and subcellular processes in the myocardium which affect contractility (Porta et al, 1983; Jensen et al, 1984; Mettler et al, 1977; Czarnecki et al, 1980; Gwathmey et al, 1983; Unverferth et al, 1984).

Cardiomyopathy induced by streptozotocin or alloxan has been studied more extensively. Both substances selectively destruct pancreatic betacells, causing diabetes mellitus (Ganda et al, 1976). Diabetes is associated with cardiomyopathy in man as well. Besides structural, endocrine and metabolic changes (Ganguly et al, 1986; Bhimji et al, 1986), Penpargkul et al (1980) found that hearts of diabetic rats showed diminished cardiac output and stroke work at high filling pressures which was not due to restrictions in coronary flow or myocardial oxygenation. Similar results were obtained later by Rodriques et al (1987), Tahiliani et al (1986) and Rösen et al (1986) while the latter study also reported reduced relaxation. Chronic (Bhimji et al, 1986; Ganguly et al, 1986) but not acute insulin therapy (Penpargkul et al, 1980; Rösen et al, 1986; Tahiliani et al, 1986) can normalize most of the alterations. Therefore, the suppression of cardiac function after streptozotocin or alloxan, is regarded as the result of a more general defect in energy provision and utilization (Rösen et al, 1986) rather than due to an impaired glucose metabolism or a direct toxic effect of the drugs. 
Table 1.3. Experimental models of heart failure; primay causes

\author{
Cardiomyopa thy \\ - spontaneous \\ - drug-induced \\ - humoral or vitamin deficiency \\ Increased afterload \\ - experimental hypertension \\ - aortic constriction \\ Increased preload \\ - fluid overload \\ - arteriovenous shunt \\ Myocardial ischemia/infarction \\ - chronic hypoxia \\ - cardiac pacing \\ - coronary artery ligation \\ - local infarction \\ - diffuse infarctions
}

Since hypertension is present in 40-80\% of patients with long-standing diabetes mellitus (Factor et al, 1981) in rat models experimental diabetes has been combined with experimental hypertension. Similar cardiac and vascular lesions were found in these models compared to patients with both hypertension and diabetes. Effects of diabetes are more pronounced in the presence of hypertension (Factor et al, 1981). Hearts of rats with renal hypertension and diabetes showed a slower contraction and relaxation with preserved peak developed tension (Fein et al, 1984). Effects disappeared after chronic treatment to normalize serum glucose and blood pressure. Thus, rats treated with alloxan or streptozotocin may provide a suitable model for heart failure as a result of diabetes induced cardiomyopathy.

Most of the models for cardionyopathy have in common that the substance that is used is not cardioselective. For drugs like adriamycin, cardiac effects are (only) adverse side-effects. The non-cardiac effects could complicate the syndrome of heart failure. Although the models may reflect the specific cardiomyopathy in man, these are not the models of first choice for the more common forms of heart failure. In addition, because some of the drugs specifically interact with the myocardial contractile system, studies with positive inotropics may be difficult to interpret. Studies on the effects of diuretics and vasodilators, however, remain possible.

Hereditary cardiomyopathy has been described in substrains of the spontaneously hypertensive rats (Ueda et al, 1982; Yamori et al, 1982), in turkeys (Einzig et al, 1980) and in syrian hamsters (Gertz, 1972). The latter is the most extensively studied. In the hamster model, the final state of the disease is characterized by ventricular dilatation and animals gaining weight through generalized fluid accumulation. "The final state is preceded by a stage of cardiac lesions and a stage of hypertrophy suggesting a normal course of development of heart failure (Gertz, 1972). Data on cardiac 
function are conflicting (Gertt, 1972; Forman et al, 1972). Hemodynamic measurements show in the pre-edematous stage bradycardia and reduced filling pressures to both ventricles resulting in a $33 \%$ decrease in cardiac output, whereas in the edematous stage filling pressures as well as stroke volume and cardiac output are increased (Abelman et al, 1973). The authors concluded that inherited cardiomyopathy in the syrian hamster results in a high output failure. The model therefore is not useful in the context of this thesis.

Similarly, increased preload in rats produced by chronic arteriovenous shunt (Flaim et al, 1979; Flaim, 1982; Noma et al, 1988) results in high output failure. Although such models eventually exhibit the consequences of heart failure, the development of the disease is different from that of low output failure.

Chronic pressure overload can be achieved by all models of chronic hypertension. Spontaneously hypertensive rats (SHR) have the advantage that no surgical intervention is needed. In isolated hearts from 78 weeks old SHR, Friberg et al (1985) found a depressed ventricular performance at high afterload when compared to hearts of 16 weeks old SHR. The study also demonstrates the importance of preload and afterload for cardiac performance. Pfeffer et al (1976, 1979b) actually made cardiac function curves in open-chest anesthetized SHR of different ages. Stulies indicate development of heart failure in SHR after approximately 18 months of age. Structural changes such as hypertrophy and increased chamber stiffness as well as intracellular biochernical alterations (Pfeffer et al, $1979 \mathrm{c}$; Tomanek et al, 1982; Engelman et $a l_{2}$ 1987) are comparable to those described in man. Thus old SHR may provide a model for heart failure after chronic hypertension in man.

Myocardial ischemia and infarction in rats is widely used as a model for heart failure (review, Curtis et al, 1987). Usually, the left descending coronary artery is occluded. Presence or absence as well as type of anesthesia, acute surgery and differences in time following ligation may contribute to the discrepancies in effects found after coronary artery ligation (Botting et al, 1983; Drexler et al, 1986b, 1987a,b,c; Pfeffer et al, 1979a, 1987). Nevertheless, in infarcted anesthetized open-chest rats, Pfeffer et al (1979a) demonstrated a depression of the cardiac function curve, which was related to the size of the infarct. In conscious infarcted rats, after cardiac stimulation by physical exercise, a depressed cardiac index was found when infarcts were healed (Drexler et al, 1986b). Although the etiology of infarction is different from that in man, the consequences of the loss of vital myocardium may be comparable. Moreover, because the infarct is induced in healthy myocardium, cardiac compensatory mechanisms can be studied separately from possibly underlyüng cardiac diseases. Thus, the model of coronary artery ligated rats may provide a model for heart failure developed after myocardial infarction.

In conclusion, depressed cardiac function has been shown in three rat models: diabetes, chronic hypertension and myocardial infarction. Since chronic hypertension and myocardial infarction are the major causes of congestive heart failure in man, models based upon these causes are further explored in the conscious rat in this thesis. 


\subsection{The present thesis}

The purpose of the present thesis is to develop a rat model to study pathophysiology and treatment of heart failure. The complexity of the syndrome implies the use of intact conscious rats, although studies in anesthetized rats and in in-vitro experiments can contribute to answer specific questions.

The pathophysiology of heart failure has been studied in two possible models, based on the major conditions leading to theart failure in man: chronic hypertension and myocardial infarction (chapter 3 and 4). Because cardiac dysfunction is the central problem in heart failure, the cardiac function curve is used as a major determinant of cardiac performance in heart failure. However, a broad variety of parameters is measured to obtain more insight in the dynamic interplay of the different mechanisms (chapter 2).

Effects of therapy have been studied, using three types of agents which are known to improve hemodynamics in patients but presumably have different effects on cardiac remodeling. On the one hand, this was performed to evaluate the clinical significance of the model by comparing effects to clinical responses, but, on the other hand, to investigate whether different effects on cardiac compensatory mechanisms may result in different hemodynamic effects. Hemodynamic effects of acute and 2 weeks treatment are studied for milrinone, which is not known to affect cardiac remodeling, for dobutamine, which may stimulate cardiac hypertrophy, and for captopril, which can regress cardiac hypertrophy and attenuate ventricular dilatation (chapters 5, 6, and 7).

Therapy described above was studied during a rather stable period in the pathophysiology of heart failure. Beneficial effects on cardiac adaptive processes imply an early intervention, i.e. before cell death occurs. Accordlingly, effects of earlier captopril were investigated and compared to the effects of the later intervention as described above (chapter 7).

Finally, data obtained in the different studies in this thesis will be compared and consequences will be discussed. The animal models are evaluated for the aim to study the pathophysiology and therapy of heart failure (chapter 8). 

chapter 2

\section{MATERIALS AND METHODS}

\subsection{Animals}

In all experiments described in this thesis, male adult rats were used. In the study described in chapter 3, spontaneously hypertensive rats (SHR) and normotensive Wistar-Kyoto rats (WKY) at ages ranging from 3 to 24 months were used. These rats were obtained from the original SHR and WKY strains bred at the Central Animal Facilities of the University of Limburg, Maastricht, The Netherlands. The other studies were performed in normotensive adult Wistar rats (Winkelmann, Borchen, Germany), weighing between 250-350 gram at the start of the experiments.

Before surgery, animals were housed in groups of up to 5 rats whereas after surgery rats were housed separately with free access to standard lab food (Hope Farms, type RMH-TM, Woerden, The Netherlands) and tap water.

\subsection{Coronary artery ligation}

\subsubsection{Chronic ligation}

Myocardial infarction was produced by ligation of the left descending coronary artery using a modification of the method of Fishbein et al (1978; Pfeffer et al, 1979a). Animals were anesthetized with pentobarbital $(60 \mathrm{mg} / \mathrm{kg}$ i.p.) and the trachea was intubated (PE-240). Skin and muscles overlying the 4th left intercostal space were separated and cut. Prophylactically, $2 \mathrm{mg} / \mathrm{kg}$ lidocaine was given i.rn. (Botting et al, 1983; Lepràn et al, 1983). The thorax was opened after starting positive pressure respiration and the heart was carefully pushed to the left or exteriorized by applying pressure to the right side of the thorax. A silk (6-0) suture was looped under the left descending coronary artery near the origin of the pulmonary artery. In sham animals the suture was looped through the myocardium next to the left descending coronary artery. When the heart was returned to its normal position, the suture was tied and ribs were pulled together with 3-0 silk. When the thorax was closed, muscles and skin were sutured separately and subatmospheric pressure was restored by inserting a silastic drain through a stab wound between the 6th and 7th rib and application of a negative pressure of $10 \mathrm{~cm} \mathrm{H}_{2} \mathrm{O}$. 


\subsubsection{Acute ligation}

Animals were anesthetized with pentobarbital $(60 \mathrm{mg} / \mathrm{kg}$ i.p.) and the trachea was intubated (PE-240). Skin and muscles overlying the 4th left intercostal space were separated and cut. The thorax was opened after starting positive pressure respiration and the heart was carefully pushed to the left or exteriorized by applying pressure to the right side of the thorax. A silk (6-0) suture was looped under the left descending coronary artery near the origin of the pulmonary artery and the heart was returned to its normal position. In sham animals the suture was looped through the myocardium next to the left descending coronary artery.

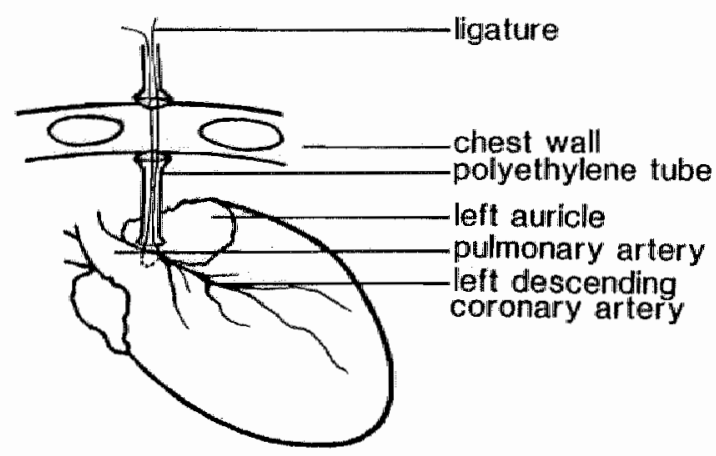

Fig. 2.1 Preparation for coronary artery ligation in the conscious rat

The suture was prepared for occlusion at a later occasion, using a modified technique of Lepràn et al (1983; fig. 2.1). Both ends of the suture were passed through the lumen of a preshaped polyethylene (PE-100) cylinder. The polyethylene cyllinder was placed between the heart and the ribs. Both ends of the ligature were left outside the thorax and the ribs were pulled together with 3-0 silk. Then, the ends of the suture were tied together with a piece of 3-0 silk without pulling. The 3-0 silk was inserted into a $9-\mathrm{cm}$ piece of $\mathrm{PE}-100$ and fixed with a metal obturator. This tube was guided subcutaneously to the neck, where it was exteriorized. The suture extended from the tube, so that, by pulling it at a later occasion, the left descending coronary artery could be occluded.

\subsection{Infarct size measurements}

After anesthetizing the rats witl pentobarbital, hearts were arrested in diastole with $1 \mathrm{M} \mathrm{KCl}$ i.v. The hearts were excised, washed in saline, vessels and atria removed and the ventricles weighed. The hearts were quickly frozen $\left(-80^{\circ} \mathrm{C}\right)$ to be cut into slices of $1 \mathrm{~mm}$ from apex to base. The slices were stained with nitro blue tetrazolium (NBT) according to Lepràn et al (1983). Briefly, the slices were incubated in $1 \mathrm{mg} / \mathrm{ml}$ NBT in $0.1 \mathrm{M}$ Sörensen phosphate buffer $(\mathrm{pH}=7.4)$ at $37^{\circ} \mathrm{C}$ for 
approximately $15 \mathrm{~min}$, and subsequently placed in distilled water. This procedure stains all tissue that was vital at the time of death. Thus it gives no information about the area at risk. From each slice, free wall of the right ventricle was removed. When infarct size was measured less than 30 hours after ligation (study of acute effects of infarction) the pale part of the slices, indicating the irreversibly damaged part of the ventricle, was cut out, and infarcted and spared left ventricle were weighed separately. Infarct size is expressed as percentage of left ventricular mass. However, with increasing age of the infarct, the infarcted part of the left ventricle becomes thinner, suggesting an underestimation of infarct size if expressed as percentage of left ventricular mass. Therefore, color pictures were taken from the left ventricle slices of those hearts, and the lengths of the infarcted area and of the spared muscle for both the endocardial and epicardial surfaces of each slice were determined by planimetry. The method for these measurements was described in detail by Pfeffer et al (1985b). Briefly, from each slice were measured: total epicardial circumference (Tep), length of infarcted epicardial circumference (Iep), total endocardial circumference (Ten) and length of infarcted endocardial circumference (Ien). For all slices of one heart, Tep, lep, Ter and len were summarized. Final infarct size was expressed in percentage of left ventricular circumference, calculated as the average of infarct size of endocardial and epicardial surfaces.

$$
\left[\frac{\Sigma(\text { Iep })}{\Sigma(\text { Tep })}+\frac{\Sigma(\text { Ien })}{\Sigma(\text { Ten })}\right] / 2 * 100 \%=\text { infarct size }
$$

Comparing both methods in four $24-$ hr old infarcted hearts resulted in infarct sizes of $8,54,10$ and $59 \%$ according to the first method, and $10,46,11$ and $57 \%$ according to the latter method. This indicates that when thinning of the wall has not yet started, both methods result in similar infarct sizes.

\subsection{Preparations for hemodynamic measurements}

\subsubsection{Cardiac output}

Cardiac output in conscious rats was measured according to the method described by Smith and Hutchins (1979) and Smits et al (1982). Rats were anesthetized with pentobarbital $(60 \mathrm{mg} / \mathrm{kg}$ i.p.) and the trachea was intubated (PE-240). "The skin and muscles overlying the 3rd right intercostal space were separated and cut. After starting positive pressure respiration (60 breaths/min, tidal volume $2.5-3 \mathrm{ml}$ ), the thorax was opened. The ribs were spread with a miniature retractor and the aorta was prepared free. An electromagnetic flow probe with a suitable diameter size (2.5-3.3 mm; Skalar, Delft, The Netherlands) was placed around the ascending aorta just above the heart. The thorax was closed by pulling the ribs together with 3-0 silk and the probe cable was fixed to the ribs. Muscles were sutured separately with 5-0 silk. The probe cable was guided to the neck where the connector was exteriorized. 

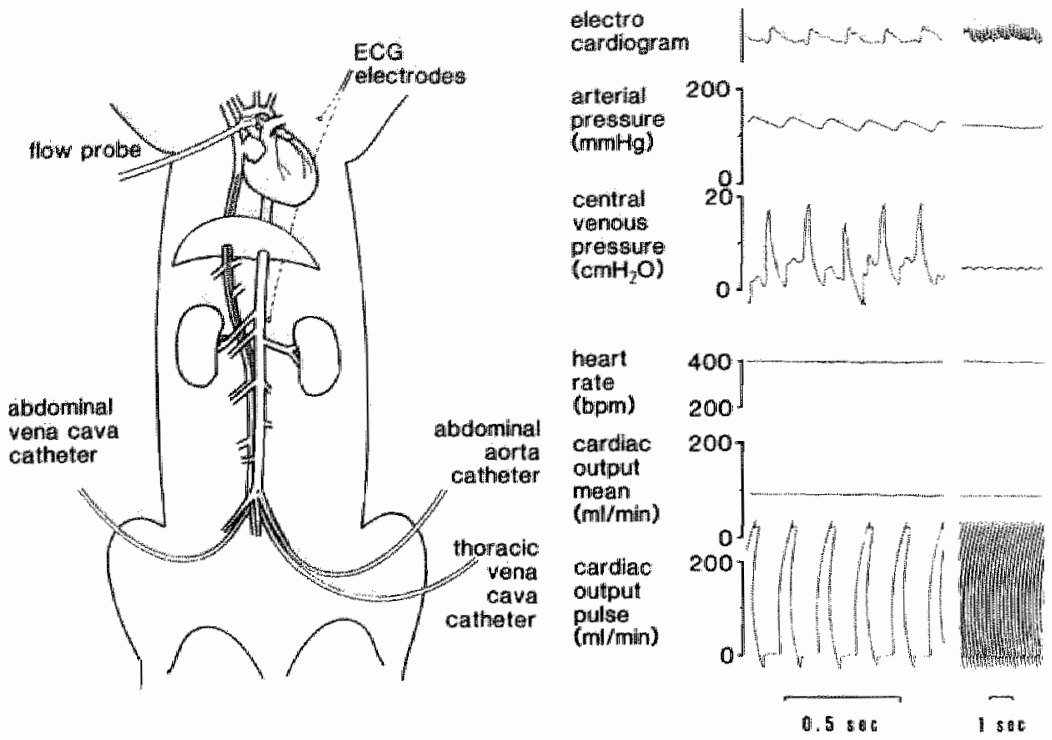

Fig. 2.2 Lay-out of the basic preparation

The skin was sutured and subatmospheric intrathoracic pressure was restored by inserting a silastic drain between the 6th

and 7 th right rib and application of a negative pressure of $10 \mathrm{~cm} \mathrm{H}_{2} \mathrm{O}$. The surgery was carried out under aseptic conditions. Rats were allowed 4-6 days for recovery.

\subsubsection{Arterial blood pressure}

The technique for chronic measurement of blood pressure in conscious rats has been extensively described by Smits (1980). The catheter was constructed from a $9-\mathrm{cm}$ piece of PE-10 tubing, heat-sealed to a $12-\mathrm{cm}$ piece of PE-50 tubing. Two small rims were made at the connection to facilitate securing of the catheter. On the other end of the PE-50 tubing, a $1-\mathrm{cm}$ piece of PE-100 tubing was heat-sealed and at $5-\mathrm{cm}$ piece of vinyl tubing (Serva TT 63) slid over it. Finally, the PE-10 tubing was bent into a J-shape by dipping it into near-boiling water. This catheter was implanted in the abdominal aorta by an approach via either the left or right femoral artery. Under light ether anesthesia, a small incision was made in the groin and the femoral artery was freed from connective tissue. After the artery was clamped with silk sutures, a small hole was cut in it and the PE-10 catheter inserted. It was advanced for $4 \mathrm{~cm}$ into the artery so that its tip was approximately $1 \mathrm{~cm}$ above the bifurcation and below the renal arteries. The catheter was secured to the artery and to the underlying muscle with silk and guided subcutaneously to the neck where it was anchored and 
exteriorized. The catheter was filled with heparinized $0.9 \% \mathrm{NaCl}$ (5 IU heparin/ml) and closed with a metal plug. Following implantation of catheters and electrodes rats were allowed at least one day for recovery.

\subsubsection{Central venous pressure}

The catheter for central venous pressure measurements was constructed of a $10-\mathrm{cm}$ piece of silastic (602-175) tubing slid over a $15-\mathrm{cm}$ piece of PE-50 tubing. Two small rims were made at a point $12 \mathrm{~cm}$ further on the PE-50 tubing. Under light ether anesthesia, a small incision was made either in the right or left groin of the rat. After the femoral vein was carefully dissected free from the surrounding tissue and clamped with silk sutures, the end of the catheter was inserted through a small hole and advanced for $9 \mathrm{~cm}$ into the vena cava which locates its tip in the thoracic vena cava just below the right atrium. The catheter was secured with silk and exteriorized in the neck in the same way as described for the arterial pressure catheter. The catheter was filled with a $0.9 \% \mathrm{NaCl}$ solution.

\subsubsection{Intravenous administration}

The venous catheter was constructed of a 9-cm piece of PE-10 tubing, heat-m sealed to a $15-\mathrm{cm}$ piece of PE-50 tubing. Two small rims were made at the connection and at a point $12 \mathrm{~cm}$ further on the PE-50 tubing. Under light ether anesthesia, a small incision was made either in the right or left groin of the rat. After the femoral vein was carefully dissected free from the surrounding tissue and clamped with silk sutures, the end of the catheter was inserted through a small hole and advanced for $4 \mathrm{~cm}$ into the vena cava. The catheter was secured with silk and exteriorized in the neck in the same way as described for the arterial pressure catheter. The catheter was filled with a $0.9 \% \mathrm{NaCl}$ solution.

\subsubsection{Electrocardiogram}

Electrodes for electrocardiogram (ECG) recording were constructed of two, teflon-coated, silver or stainless steel wires (AS 766, AS 633, Cooner Wire Company, Chatsworth, CA, USA) with lengths of 8 and $14 \mathrm{~cm}$, respectively. They were soldered to a miniature connector. This connector was passed through a $3-\mathrm{cm}$ piece of PE-270 tubing filled with silicon rubber, preventing traction on the connection and avoiding contact with fluid. This was further closed using a $4-\mathrm{cm}$ piece of crimp tubing. At the end, both wires were freed from teflon over $0.5 \mathrm{~cm}$. Under light ether anesthesia, a small incision was made at the neck and another at the lower part of the back, $3 \mathrm{~cm}$ above the tail origin. Electrodes were guided subcutaneously so that the long one was over the sacral region whereas the short one was over the right scapula. The connector was secured and exteriorized in the neck. 


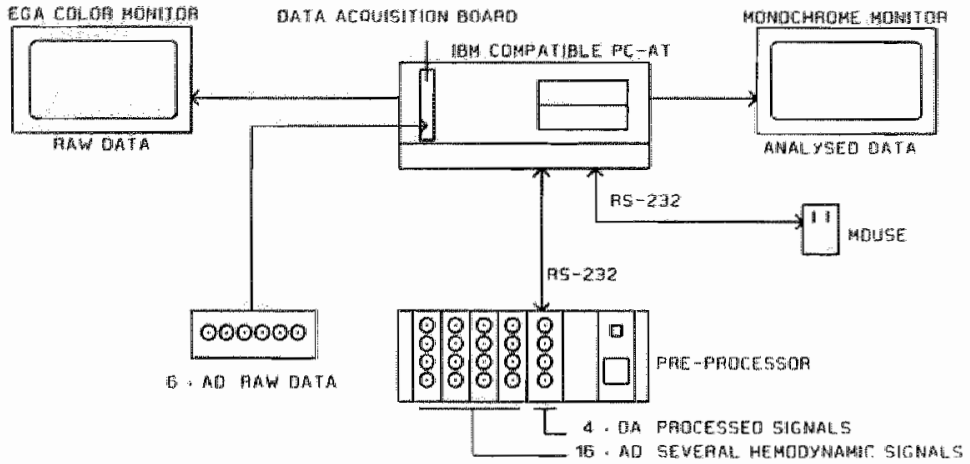

Fig. 2.3 Scheme of the "real-time data acquisition" system

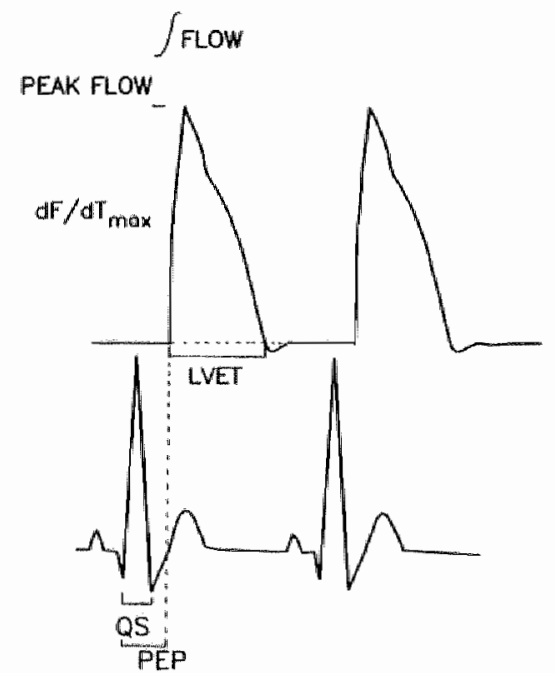

Fig. 2.4 Derived hemodynamic parameters. $d F / d t$ max: maximal flow acceleration; LVET: left ventricular ejection time; QS: QS width; PEP: pre-ejection period 


\subsection{Hemodynamic measurements}

After recowery from surgery, the rats were placed in plastic experimental cages $(22 \times 13 \times 13 \mathrm{~cm})$ on sawdust, and connected to the measuring equipment. The flow probe was connected to a sine-wave electromagnetic flow meter (Skalar, Delft, The Netherlands). The catheters for arterial and central venous pressure measurements were connected to miniature low volume displacement pressure transducers (CP-01, Century Technology Company, Inglewood, CA, USA), whereas the other venous catheters were connected to a piece of PE-50 tubing for intravenous administration. The electrodes were connected to an ECG amplifier. The signals were recorded with a Grass model 7D polygraph (Grass Instruments, Quincy, Mass, USA). The flow signal was displayed on the recorder and zero flow was taken to be the late horizontal part of diastole since the probe was situated just above the heart. Cardiac output was obtained from the electronic mean of the flow signal. Heart rate was determined from the pulsatile signal by a tachograph (Grass, 7D polygraph). Mean arterial pressure and mean central venous pressure were obtained by low-pass filtering ( 0.5 $\mathrm{Hz}$ ) of the pressure signals.

Signals from the ECG electrodes were amplified differentially. The rats were allowed to habituate for at least $45-60$ min before recording hemodynamic variables. An outline of the basic preparation in an infarcted rat is shown in fig. 2.2.

Along with the development of animal models for heart failure, a growing need was felt for more detailed information about the different hemodynamic parameters than mean values could provide. Since lowered cardiac output is regarded as the crucial factor in heart failure, special attention was paid to the flow signal. Although from a technical point of view it may be possible to derive aortic flow acceleration, maximal aortic flow and stroke volume by analog differentiation or integration of the flow signal, registration of full amplitudes of the pulsatile signals would be limited by the mechanical way of recording because of the relatively high heart rate of rats. A second problem was comparing time courses of different signals to each other. Therefore, at the department of Instrumental Serivices of the University of Limburg (Maastricht, The Netherlands) a real-time data processing system was developed. Briefly, this system encompasses a 68B09-based preprocessor, an AT compatible microcomputer and two monitors (fig. 2.3). This system samples hemodynamic signals at $500 \mathrm{~Hz}$ and plots them in real time according to user-selected mathematical methods, thereby instantaneously providing relevant data. Parameters can be presented graphically, numerically, or both. In order to analyze hemodynamic data after ending the experiment, the data were stored on hard disk. Furthermore, the system is capable of on-line graphical monitoring of non-processed sensor signals to enable direct visual inspection of the waveform of flow and pressure signals and the shape of the $\mathbb{E C G}$ signal. For detailed waveform analysis, storage of one minute periods of the $500 \mathrm{~Hz}$ sampled sensor signals is possible.

From the flow signal, cardiac output $(\mathrm{CO})$, heart rate (HR), artic flow acceleration (dF/dt), peak aortic flow (PF), stroke volume (SV) and duration of the flow pulse (left ventricular ejection time: LVET) were derived (fig. 2.4). Systolic, diastolic and mean arterial pressure (MAP) were recorded from the arterial pressure signal whereas mean central venous pressure (CVP) was recorded from the central 


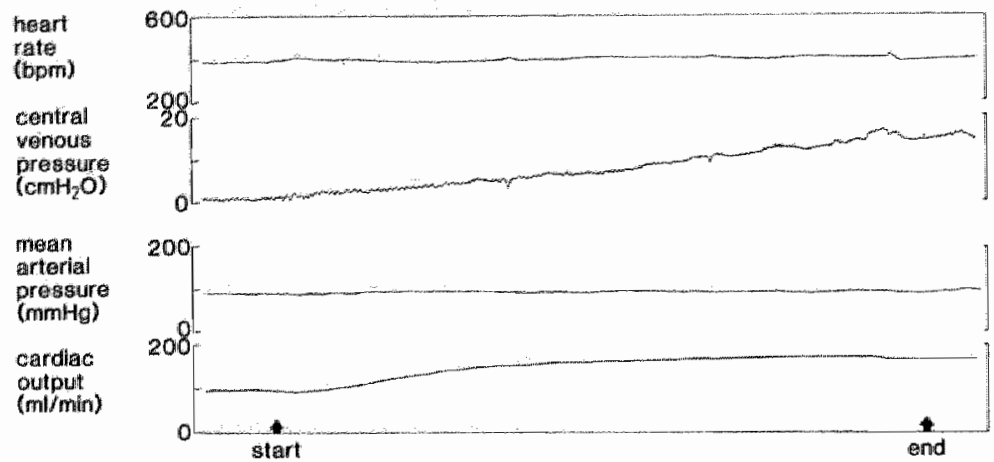

Fig. 2.5 Typical recording of a volume load; $12 \mathrm{ml}$ Ringer's solution in one minute. Arrows define start and end of the volume load

\section{CARDIAC FUNCTION CURVE}

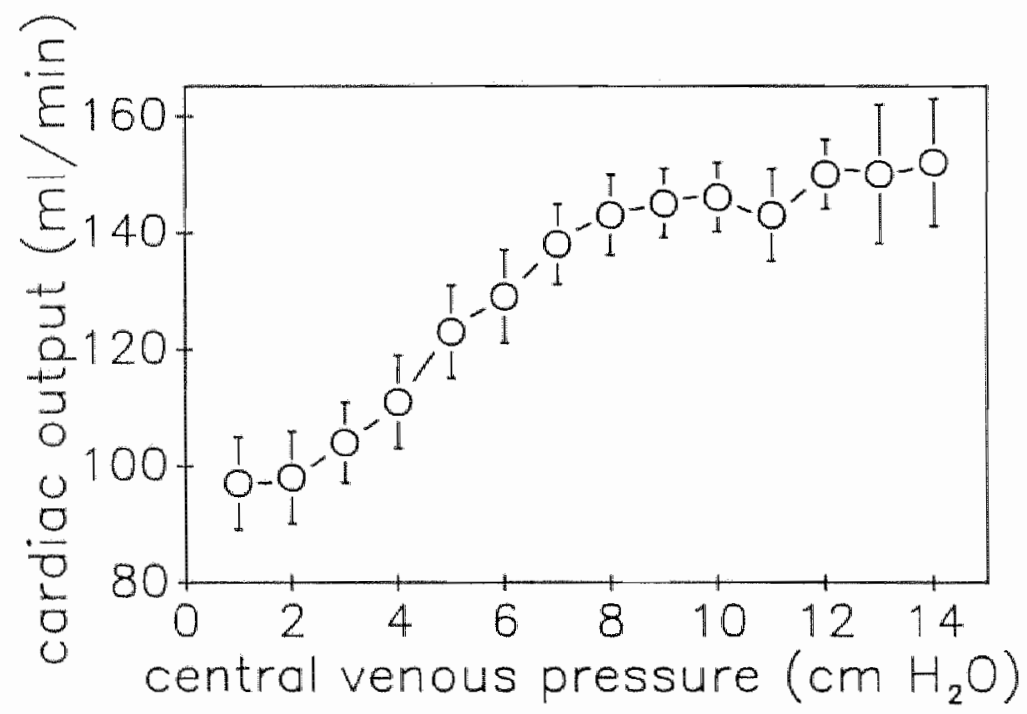

Fig. 2.6 Cardiac function in normal healthy rats (mean $\pm S E M ; n=11$ ) 
wenous pressure signal. Total peripheral resistance (TPR) was computed as the difference between MAP and CVP divided by CO. From the ECG, the duration of the QS interval was determined. The time period between the Q peak in the ECG wnd the start of the following aortic flow pulse was taken as the pre-ejection period (PEP) (fig. 2.4) and the ratio PEP/LVET was computed Stroke work (SW) was estimated by multiplying SV with the difference between MAP and CVP (Pfeffer et all, 1979a,b,c). All parameters were registered continuously and, for later analysis, stored on a hard disk.

\subsubsection{Method for quantifying heart failure}

Although heart faillure may be present in animals, cardiac function could appear to be normal at rest. Failure may become overt only when an extra demand is made. The most physiological way to stimulate cardiac output is by means of physical exercise. Using physical exercise in a model of rats with myocardial infarction, heart failure was demonstrated by a significantly reduced cardiac index (Drexler et al, 1986b). Obviously, however, this method is very complicated in instrumented rats. An alternative is to determine the relationship between cardiac filling pressure and cardiac output. Such a method can be based upon a rapid intravenous volume loading. Pfeffer et al (1979a) developed this alternative to quantitate heart failure in anesthetized rats with myocardial infarction. Comparing results from this study (Pfeffer et al, 1979a) to the study of Drexler et al (1986b) indicates that volume loading may offer a good alternative to demonstrate heart failure in chronically instrumented infarcted rats.

Although this method is used extensively in anesthetized rats to demonstrate heart failure (Pfeffer et al, 1979a,b; 1982a; 1985a,b; 1987), no studies are available on this method in conscious rats. In anesthetized rats, infusion of warm $\left(39^{\circ} \mathrm{C}\right)$ Tyrode's solution ( $40 \mathrm{ml} / \mathrm{min} . \mathrm{kg}$ for 45 seconds) produced a rise in cardiac output to a plateau level despite further increase of right atrial pressure (Pfeffer et al, 1979a,b,c). Several experiments were performed to evaluate this method in conscious rats and these will be summarized briefly.

\subsubsection{Evaluation of method for quantifying heart failure}

In the first place, effects of volume loading with different fluids were compared. Therefore, a rapid infusion of either heparinized blood, plasma, "Tyrode's solution (137 $\mathrm{mM} \mathrm{NaCl}_{2}, 2.7 \mathrm{mM} \mathrm{KCl}, 1.8 \mathrm{mM} \mathrm{CaCl}_{2}, 1.1 \mathrm{mM} \mathrm{MgCl}, 11.9 \mathrm{mM} \mathrm{NaHCO}, 0.4$ $\mathrm{mM} \mathrm{NaH} \mathrm{PO}_{4}$, and $5.6 \mathrm{mM}$ glucose) or Ringer's solution (154 mM NaCl, $2.7 \mathrm{~mm}$ $\mathrm{KCl}_{3} 1.8 \mathrm{mM} \mathrm{CaCl}$, and $1.2 \mathrm{mM} \mathrm{NaHCO}$ ) was applied and $\mathrm{CO}$ was nonitored continuously in the conscious rat. Obviously, all fluids were at body temperature $\left(37^{\circ} \mathrm{C}\right)$ before infusion. The infusion of blood was found to be troublesone, because of clotting. Similar problems were found when plasma was used. "The turbulence caused by the rapid infusion probably resulted in denaturation of proteins. However, CO response to this infusion was an increase of $40 \pm 11 \mathrm{ml} / \mathrm{min}(\mathrm{n}=4)$. Comparable CO responses were found after infusion of Tyrode's solution: $43 \pm 9 \mathrm{ml} / \mathrm{min}(\mathrm{n}=3)$ and Ringer's solution: $45 \pm 6 \mathrm{ml} / \mathrm{min}(\mathrm{n}=6)$. From these experiments, infusion of 
$12 \mathrm{ml}$ Ringer $\mathrm{r}^{\prime} \mathrm{s}$ solution $\left(37^{\circ} \mathrm{C}\right)$ in 60 seconds was chosen for the volume load.

A second point was that the volume load was given before the right atrium whereas the output was measured from the left ventricle neglecting any effect of the pulmonary circulation. To verify this, wolume loading through the left atrium was compared to volume loading by intravenous infusion. For that purpose, an additional catheter (silastic tubing) was placed in the left atrium via a left thoracotomy, followed by at least 4 days of recovery. The former procedure increased $\mathrm{CO}$ with $39 \pm 6 \mathrm{ml} / \mathrm{min}$ while CVP was elevated from $2.1 \pm 0.7$ to $17.5 \pm 2.3 \mathrm{~cm} \mathrm{H}_{2} \mathrm{O}(\mathrm{n}=7)$, whereas the latter method increased $\mathrm{CO}$ with $35 \pm 5 \mathrm{ml} / \mathrm{min}$ while CVP rose from $2.8 \pm 2.2$ to $16.3 \pm 2.4 \mathrm{~cm} \mathrm{H}_{2} \mathrm{O}(\mathrm{n}=5)$. Left atrial pressure, measured in different rats, showed comparable increases in both groups (from $8.5 \pm 1.2$ to $25.5 \pm 1.4 \mathrm{~cm} \mathrm{H}_{2} \mathrm{O}$ $(\mathrm{n}=2)$ and from $7.2 \pm 1.3$ to $23.9 \pm 3.1 \mathrm{~cm} \mathrm{H}_{2} \mathrm{O}(\mathrm{n}=4)$ in left atrium loaded and intravenously loaded groups, respectively). These results suggest that no differences in cardiac response to volume load are found when this load is given proximally or distallly to the pulmonary circulation in normal healthy rats, but may differ in rats with heart failure. In right heart failure, the right ventricle would be the limiting factor for the $\mathrm{CO}$, measured distally to the left ventricle. However, in the models described in this thesis, the left ventricle will be the limiting factor for $\mathrm{CO}$. Since implantation of a left atrium catheter would imply another thorax surgery volume loading was given as an intravenous infusion.

In order to investigate whether activation of sympathetic reflex mechanisms would depress maximal response, volume load was given to baroreceptor denervated rats, according to a method described by Krieger et al (1967) and to intact rats. Although resting $\mathrm{CO}$ was decreased by the denervation, responses to volume loading were comparable: $37 \pm 5 \mathrm{ml} / \mathrm{min}(\mathrm{n}=6)$ in denervated rats and $38 \pm 7 \mathrm{ml} / \mathrm{min}(\mathrm{n}=6)$ for intact rats. When neural regulation was completely blocked by cholinergic (atropine) and adrenergic (propranolol) blockade comparable results were found, suggesting that cardiac response to volume loading may be independent of neural regulation.

In conclusion, the procedure of volume loading by a rapid intravenous infusion could be performed in conscious rats, resulting in a highly reproducible increase in cardiac output. The cardiac response to volume loading is found to be independent of the site of infusion and of the autonomic nervous system. Therefore, a rapid intravenous infusion of $12 \mathrm{ml}$ warm $\left(37^{\circ} \mathrm{C}\right)$ Ringer's solution in 60 seconds was chosen to determine cardiac performance. A typical recording of this volume loading is presented in fig. 2.5. For 11 normal healthy rats, this yielded a cardiac function curve as illustrated in fig. 2.6. This curve (Frank-Starling curve) can be characterized by its starting point (CVP and $\mathrm{CO}: 1.1 \pm 0.3 \mathrm{~cm} \mathrm{H} \mathrm{H}_{2} \mathrm{O}$ and $87 \pm 3 \mathrm{ml} / \mathrm{min}$ ) and the plateau level for the cardiac output $(143 \pm 4 \mathrm{ml} / \mathrm{min})$ which is reached during the loading while CVP is still increasing. Volume loading did not affect HR and MAP (HR: $386 \pm 7$ before and $400 \pm 5$ beats/min after volume loading; MAP: $109 \pm 3$ before and $112 \pm 2 \mathrm{~mm} \mathrm{Hg}$ after volume loading). 


\subsection{Ejection fraction measurements}

Rats were subjected to a venous catheter implantation as described in section 2.4.4. Three days later, animals were anesthetized with intravenous pentobarbitall till they just had lost the toe-pinch reflex. Rats were placed in that left anterior oblique position in which the best separation between the right and left ventricle was achieved. Then, multigated equilibrium cardiac blood pool imaging was performed using in vivo labeling of autologous red blood cells with 2 mCi of 99 mTc. This was injected intravenously in a volume of $<1 \mathrm{ml}$. Images were acquired using a Technicare mobile gamma camera with a pin hole purpose collimator, interfaced to 560 Technicare minicomputer. Acquisition time was 30 minutes with a photo peak of 140 $\mathrm{keV}$ and a window of $20 \%$. The left ventricular ejection fraction was calculated using a semi-automatic analyzing program. Analysis was blindly performed in triplo.

\subsection{Statisties}

In the following chapters different statistical methods were used to compare data in several experimental groups.

1. Student's t-test for paired observations. This method was used to compare values of the same group of rats in two different situations.

2. Student's t-test for unpaired observations. This method was used to compare maximally two groups of unpaired values.

3. One-way analysis of variance and a modified t-test for overall comparisons of the experimental groups, following the Bonferroni method and for multiple comparisons to the same control group, following Dunnett's test (Wallenstein et all, 1980). This method was used to compare more than two groups of unpaired values.

4. Linear regression analysis for determination of relationships. This method was used to correlate two independently measured values.

Data are expressed as means \pm SEM. Differences or correlations were regarded to be statistically significant if $\mathrm{p}<0.05$.

\subsection{Drugs used in this thesis}

In the following chapters different therapeutic agents were used.

Milrinone: Milrinone was received from Sterling Winthrop, Haarlem, The Netherlands.

Dobutamine: Dobutrex was obtained from Eli Lilly, Amsterdam, The Netherlands.

Captopril: Captopril was obtained as a generous gitt from Squibb, Rijswijk, The Netherlands.

Benazepril: Benazepril (CGS 14824A) was received as a generous gift from Ciba Geigy, Basel, Switzerland.

Lidocaine: Xylocaine was obtained from Astra, Rijswijk, The Netherlands. 



\section{THE AGING SPONTANEOUSLY HYPERTENSIVE RAT AS A MODEL FOR HEART FAILURE}

\subsection{Introduction}

In man, hypertension is one of the major causes of congestive heart failure. While compensating mechanisms like cardiac hypertrophy jnitially act to maintain cardiac performance against an increasing afterload, at a later stage they can develop into de-adaptation and consequent heart failure (Meerson, 1983). Several rat models for hypertension have been developed, of which genetic hypertension in spontaneously hypertensive rats (SHR) has been studied most extensively. Genetic hypertension provides the possibility of longitudinal studies of the natural progression of hypertension and related diseases without surgical interventions to induce hypertension.

Experiments with isolated hearts from SHR of different ages (Noresson et al, 1979a, Hallbäck-Nordlander et al, 1979; Friberg et al, 1985, 1986) indicate the importance of preload and afterload for cardiac performance. In hearts of old SHR, cardiac performance is reduced at high afterload. Studies in intact anesthetized open-chest SHR of different ages (Pfeffer et al, 1976, 1979b,c; Noresson et al, 1979b; Mirsky et al, 1983; Nishimura et al, 1985; Vogt et al, 1987a,b) indicate development of left ventricular failure from about 18 months of age.

The purpose of the present study is 10 investigate in conscious SHR whether chronic hypertension leads to heart failure, defined as a depression of the cardiac function curve. In other words, do old SHR provide a suitable model to study pathophysiology and therapy of heart failure?

To study hemodynamics in the conscious animal without effects of acute surgery, rats were chronically instrumented with a flow probe for $\mathrm{CO}$ measurement, catheters for MAP and CVP measurements and electrodes for ECG registration. Hemodynamics were measured not only at baseline conditions, but also after maximal stimulation of cardiac output by volume lowding.

Since spontaneous mortality strongly increases after 17 months in the used SHR strain, effects of chronic hypertension and of aging itself were investigated in 3 and 17 months old SHR and WKY with an additional group of 12 months old SHR. Hemodynamics of older SHR are difficult to interpret since these rats represent a selected group of survivors with various poorly defined physiological deficits. "There. fore, in 21-22 months old SHR and WKY ejection fractions were measured, using at method which requires less surgical intervention. 


\subsection{Experimental protocols}

Experiments were performed in male spontaneously hypertensive rats (SHR) and Wistar-Kyoto rats (WKY) at different ages. Hemodynamics of 3 months SHR and WKY were compared using a Grass 7D polygraph for hemodynamic registrations and a dual-beam digital storage oscilloscope for systolic times measurements. Because the hemodynamics of the other groups were measured using the real-time data processing system, measurements in 3 months SHR were repeated.

\subsubsection{Hemodynamic measurements}

\section{Surgery protocol}

SHR of 3,12, 17 and 20 months of age and 3 and 17 months old WKY were anesthetized with pentobarbital and an electromagnetic flow probe was implanted on the ascending aorta (cf. 2.4.1). Probe diameters varied from $2.6 \mathrm{~mm}$ in 3 months old rats to $3.3 \mathrm{~mm}$ in old SHR. Rats were allowed to recover 5-6 days from the surgery. Following the recovery period, animals were reanesthetized with ether and an arterial catheter for MAP measurements, a venous catheter for CVP measurement (except in 3 months old WKY) and 2 venous catheters for infusions were implanted (cf. 2.4.2-2.4.4). Furthermore, ECG electrodes were implanted (cf. 2.4.5). Experiments started at least one day after this surgery.

\section{Measuring protocol}

To perform hemodynamic measurements, rats were connected to the measuring equipment and signals were monitored on a Grass model 7D polygraph and a dual-beam digital storage oscilloscope or fed into the real-time data processing system (cf. 2.5) as mentioned above. Using the former method, besides MAP, HR and $\mathrm{CO}, \mathrm{dF} / \mathrm{dt}$ by differentiation of the flow signal, PF, SV, LVET, PEP and QS were obtained. After equilibration for $45-60 \mathrm{~min}$, baseline values for all variables were obtained during $15 \mathrm{~min}$. Then, $12 \mathrm{ml}$ of a warm $\left(37^{\circ} \mathrm{C}\right)$ Ringer's solution was infused in one minute. During this intervention, all hemodynamic parameters were monitored continuously. The volume load increased $\mathrm{CO}$ to its plateau level after about $45 \mathrm{~s}$, in spite of further increasing CVP. Therefore, CO over the last $10 \mathrm{~s}$ of volume loading was regarded as the maximally stimulated CO. Recovery time was defined as time after infusion in which CVP returned to its pre-infusion value or stabilized, with a maximum of $60 \mathrm{~min}$.

\subsubsection{Ejection fraction measurements}

In $21 / 22$ months old SHR and WKY a venous catheter for infusion was implanted under light ether anesthesia (cf. 2.4.4). Three days later, ejection fractions were measured (cf. 2.6). Rats were anesthetized with intravenous pentobarbital till they just lost the toe-pinch reflex. Left ventricular contraction was monitored for at least $30 \mathrm{~min}$ and stored on a disk for later analysis of ejection fraction. 


\subsection{Results}

\section{General}

Without experimental intervention, spontaneous mortality in SHR and WKY did not exceed $30 \%$ till 17 months of age, and then increased steeply in SHR. No SHR survived 2 years. The number of rats $(n)$ subjected to the protocol and mortality at different stages of the protocol are presented in table 3.1. Survival decreased with age but no differences were found between SHR and WKY till 17 months of age. However, only $20 \%$ of the older SHR survived surgery and recovery. Thoracic surgery in these rats was hampered by respiratory problems because rats were suffering from excessive pleural effusion and peripheral oedema. After successful hemodynamic studies on 5 SHR, experiments were terminated. Results are analyzed separately since these rats may represent a selected group of survivors. In older rats, just ejection fraction was measured, since this requires only minor surgical intervention, i.e. implantation of a venous catheter, which greatly improved survival (table 3.1).

Table 3.1 Montality in the experimental groups at different stages of the protocol

\begin{tabular}{lllll}
\hline & $\mathrm{n}$ & $\begin{array}{l}\text { Mortality } \\
\text { during } \\
\text { surgery }\end{array}$ & $\begin{array}{l}\text { Mortality } \\
\text { during } \\
\text { recovery }\end{array}$ & Survivors \\
\hline SHR 3 months & 10 & 0 & 0 & 10 \\
SHR 12 months & 19 & 6 & 2 & 11 \\
SHR 17 months & 17 & 4 & 2 & 11 \\
WKY 17 months & 18 & 5 & 2 & 11 \\
SHR 20 months & 25 & 17 & 3 & 5 \\
SHR 21/22 months & 6 & 0 & 0 & 6 \\
WKY 21/22 months & 6 & 0 & 0 & 6 \\
\hline
\end{tabular}

*Used only for ejection fraction measurements

At gross autopsy at the end of experiments, heart sizes increased with age in SHR and were larger than in WKY at 17 months and older. Signs of congestion in lungs and liver were seen only in SHR of 17 months or older.

\section{Hemodynamic effects}

In order to study heart failure on the basis of a cardiac function curve, baseline and stimulated CO were compared in 3,12 and 17 months old SHR and 3 and 17 months old WKY. Results are presented in fig. 3.1. From this figure, it can be seen 


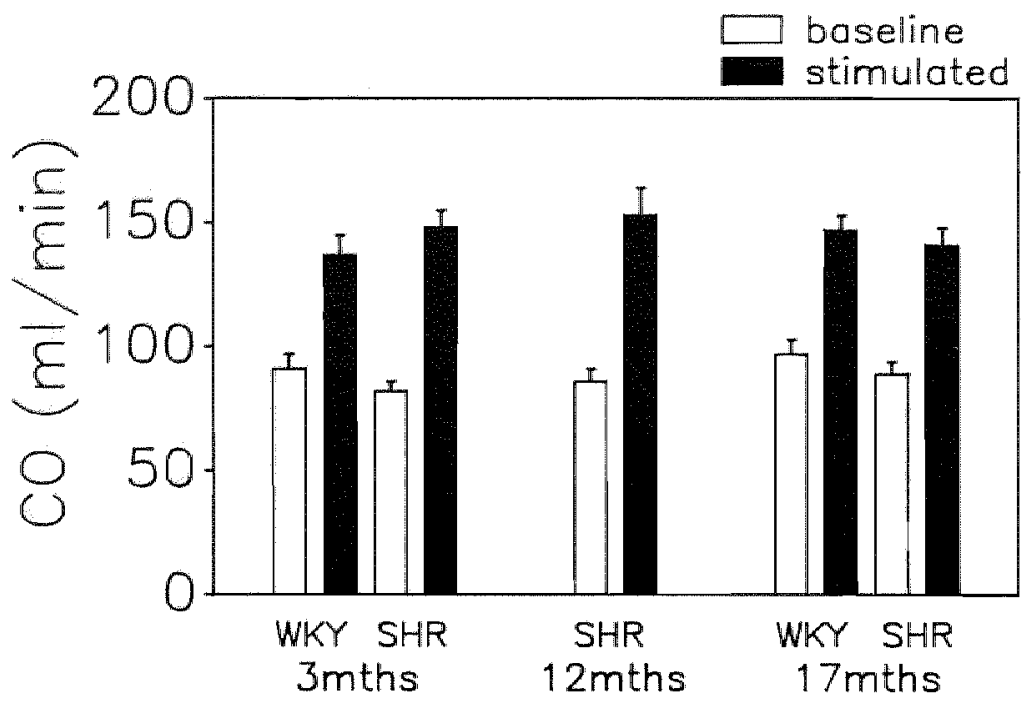

Fig. 3.1 Baseline and stimulated $C O$ at different ages in $W K Y$ and SHR

that, at least till 17 months of age, neither chronic hypertension nor aging alters cardiac performance.

At 3 months of age, SHR and WKY did not differ in any respect except for MAP, TPR and QS. No effects of aging nor of chronic hypertension were found on baseline LVET, dF/dt, PF, CVP and CI. Baseline MAP, TPR and QS were consistently lower in WKY. Baseline HR tended to decline while SV tended to increase with aging, but neither was affected by chronic hypertension (fig. 3.2). Baseline SW remained constant in WKY (SW values for 3 months WKY was estimated by SV x MAP since CVP was not measured), whereas in SHR SW increased with aging. In 17 montlss old SHR, PEP and PEP/LVET were significantly elevated (fig. 3.2), indicating reduced contractility and ejection fraction respectively. LVET in 3 months old WKY are not included in the figure, because it was not measured with the real-time data processing method. However, no differences between 3 months old SHR and WKY were found if measured with the same system (both $80 \pm 2 \mathrm{~ms}$ ).

Systolic time intervals, PEP and LVET, are discussed to be inversely related to HR (Rousson et al, 1987). Therefore, the relationship between HR and PEP and LVET were investigated using linear regression analysis. Whereas in none of the groups PEP was correlated with HR, in all groups LVET was significantly related to HR (fig. 3.3). Using the regression equation per group, LVET for HR $=0$ was calculated as LVETI. Results (table 3.2) indicate that besides the increased PEP, reduction of LVETI in 17 months SHR contribute to the elevated PEP/LVETI.

After stimulation, although peak $\mathrm{CO}$ was not depressed, impaired contractility became overt from a significantly depressed dF/dt and increased PEP. In 17 months old SHR, ejection fraction during the increased filling pressure due to volume loading 

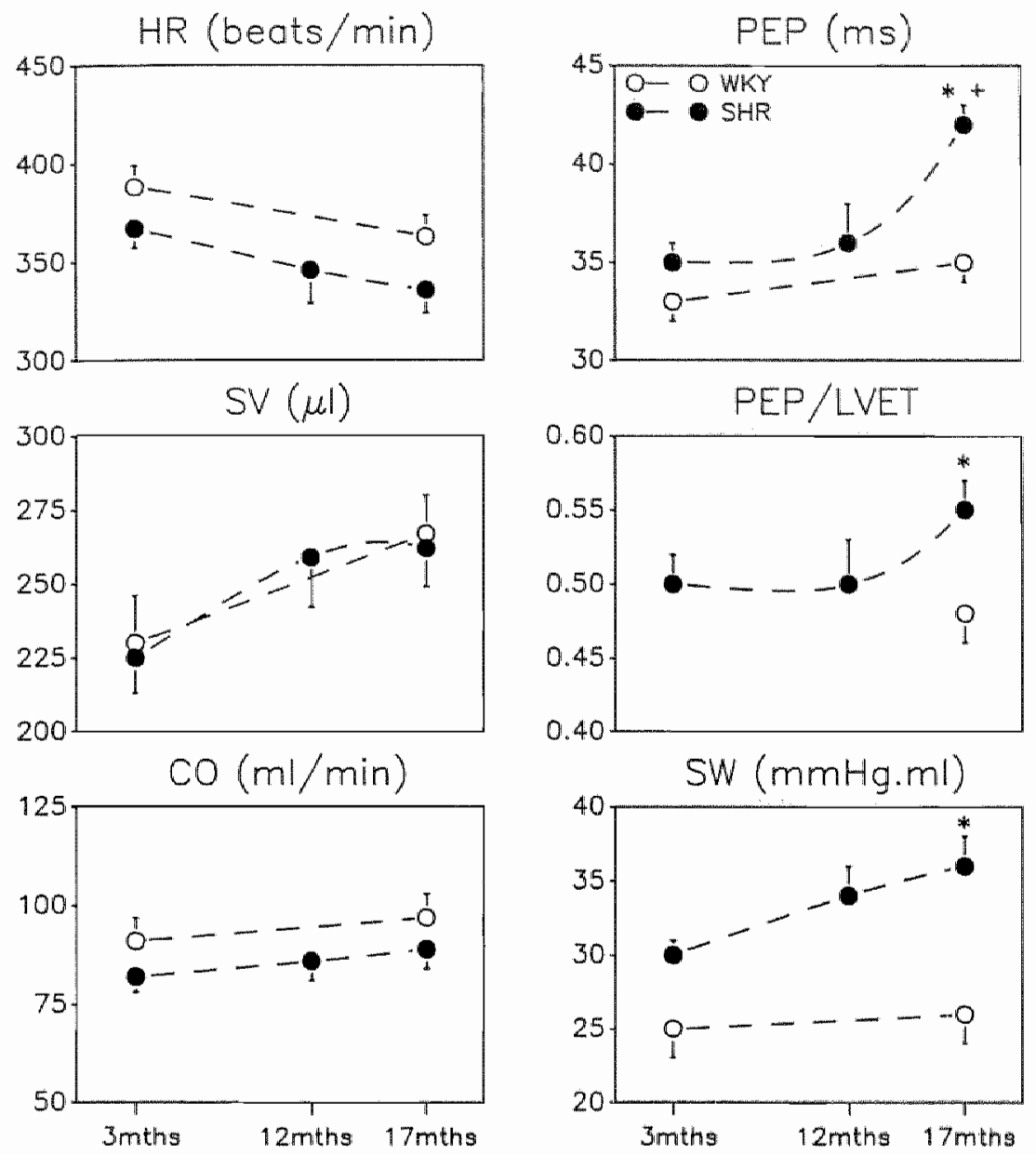

Fig. 3.2 Effects of aging and chronic hypertension on baseline hemodynamic parameters; *Significantly different from age-matched WKY; + Significantly different from 3 month SHR

was reduced, as indicated by an increased PEP/LVET, compared to both 17 months WKY and 3 months SHR (table 3,3).

Recovery time, defined as the time in which CVP after volume loading returns to baseline or stabilizes, was increased with aging in SHR (3 months SHR: $11 \pm 1$ min, 12 months SHR: $13 \pm 3 \mathrm{~min}$, and 17 months SHR: $16 \pm 3$ min) but comparable to WKY at 17 months $(17 \pm 2$ min). 

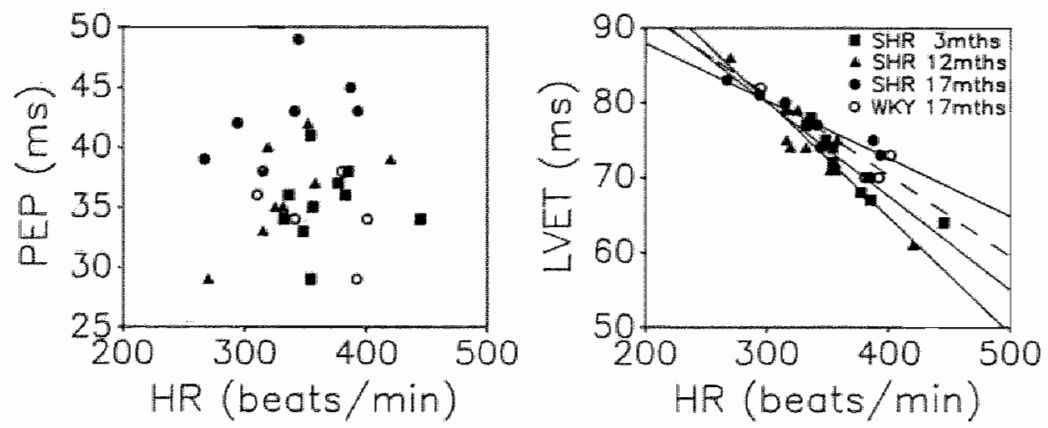

Fig. 3.3 Systolic times related to HR. Regression equations: SHR 3 months (-) LVET = $-0.13 * H R+117, r=-0.91 ;$ SHR 12 months $(-) L V E T=-0.16 * H R+127, r=$ -0.94; SHR 17 months ( - LVET $=-0.08 * H R+103, r=-0.93$; WKY 17 months (-

-) $L V E T=-0.12 * H R+114, r=-0.92$

Table 3.2 Systolic times corrected for heart rate

\begin{tabular}{lcccc}
\hline & $\begin{array}{c}\text { SHR } \\
3 \text { months }\end{array}$ & $\begin{array}{c}\text { SHR } \\
\text { months }\end{array}$ & $\begin{array}{c}\text { SHR } \\
\text { months }\end{array}$ & $\begin{array}{c}\text { WKY } \\
17 \text { months }\end{array}$ \\
\hline LVET (ms) & $72 \pm 1$ & $75 \pm 2$ & $77 \pm 1$ & $73 \pm 1$ \\
LVETI (ms) & $118 \pm 2$ & $127 \pm 2^{+}$ & $103 \pm 1^{*+}$ & $113 \pm 2$ \\
PEP/LVETI & $0.30 \pm 0.03$ & $0.29 \pm 0.03$ & $0.42 \pm 0.04^{*+}$ & $0.30_{ \pm 0}$ \\
\hline
\end{tabular}

* Significantly different from 3 months old SHR

* Significantly different from age-matched WKY

Results indicate that while cardiac performance is maintained in 17 months old SHR, this is achieved by a slightly slower beating, less contractile, larger heart. Maintained SV with reduced ejection fraction indicates ventricular dilatation.

After 17 months of age, spontaneous mortality in SHR increased severely. Hemodynamics at baseline and after volume loading of survivors with a mean age of 20 months are shown in table 3.4. When comparing these rats to the 17 months old SHR, all flow-related parameters $(\mathrm{dF} / \mathrm{dt}, \mathrm{PF}, \mathrm{SV}, \mathrm{SW}$ and $\mathrm{CO})$ were increased at baseline and after volume loading. Rats remained hypertensive. Comparable PEP at 17 and 20 months indicates similar contractility. In contrast, increased PEP/LVET indicates a further reduction of ejection fraction. Recovery time was $41 \pm 6 \mathrm{~min}$, indicating a delayed reduction of circulating volume after infusion. 
Table 3.3 Hemodynamics after stimulation by volume loading

\begin{tabular}{|c|c|c|c|c|}
\hline & $\begin{array}{c}\text { SHR } \\
\text { 3. months }\end{array}$ & 12 months & 1) months & 17 WKY \\
\hline $\mathrm{n}$ & 10 & 10 & 10 & 11 \\
\hline velght $(\mathrm{g})$ & $317 \pm 6$ & $396 \pm 11^{+}$ & $412+10^{*+4}$ & $477 \pm 11$ \\
\hline $\mathrm{dF} / \mathrm{dt}\left(\mathrm{ml} / \mathrm{s}^{2}\right)$ & $594 \pm 28$ & $593 \pm 43$ & $531 \pm 37^{*}$ & $644+28$ \\
\hline $\mathrm{PF}(\mathrm{ml} / \mathrm{min})$ & $476 \pm 21$ & $499 \pm 28$ & $466 \pm 22$ & $511 \pm 20$ \\
\hline SV $(\mu 1)$ & $360 \pm 15$ & $383 \pm 19$ & $366 \pm 15$ & $390+15$ \\
\hline $\mathrm{CO}(\mathrm{ml} / \mathrm{min})$ & $148 \pm 7$ & $153+11$ & $141 \pm 7$ & $147 \pm 6$ \\
\hline $\mathrm{HR}\left(\min ^{-1}\right)$ & $410 \pm 7$ & $405 \pm 10$ & $385 \pm 5$ & $380 \pm 4$ \\
\hline $\operatorname{CVP}\left(\mathrm{cm} \mathrm{H}_{2} 0\right)$ & $11.3 \pm 0.7$ & $9.1 \pm 0.4$ & $9.5 \pm 1.0$ & $10.5 \pm 0.7$ \\
\hline $\operatorname{PEP}(\mathrm{ms})^{2}$ & $31 \pm 1$ & $32 \pm 1$ & $39_{ \pm 1}{ }^{*+}$ & $33 \pm 1$ \\
\hline $\mathrm{PEP} / \mathrm{LVET}$ & $0.38 \pm 0.01$ & $0.42 \pm 0.02$ & $0.46 \pm 0.01^{*+}$ & $0.39 \pm 0.02$ \\
\hline $\mathrm{SW}(\mathrm{ml}, \mathrm{mmHg})$ & $46 \pm 2$ & $50 \pm 2$ & $50 \pm 2^{*}$ & $37 \pm 2$ \\
\hline
\end{tabular}

${ }_{*}^{+}$Significantly different from 3 months old SHR *Significantly different from 17 months old WKY

Table 3.4 Hemodynamics in old SHR at baseline and after volume loading ( $n=5$; mean age: 20 months)

Baseline

$\begin{array}{lcc}\mathrm{dF} / \mathrm{dt}\left(\mathrm{ml} / \mathrm{s}^{2}\right) & 641 \pm 23 & 714 \pm 11 \\ \mathrm{PF}(\mathrm{ml} / \mathrm{min}) & 527 \pm 22 & 591 \pm 10 \\ \mathrm{SV}(\mu \mathrm{l}) & 354 \pm 24 & 464 \pm 19 \\ \mathrm{CO}(\mathrm{ml} / \mathrm{min}) & 125 \pm 7 & 174 \pm 7 \\ \mathrm{HR}\left(\mathrm{min}^{-1}\right) & 352 \pm 11 & 374 \pm 1.0 \\ \mathrm{CVP}\left(\mathrm{cm} \mathrm{H} \mathrm{H}_{2} 0\right) & 2.6 \pm 1.7 & 15.3 \pm 1.9 \\ \text { MAP (mmHg) } & 131 \pm 1.2 & 137 \pm 10 \\ \mathrm{TPR}(\mathrm{mmHg} \cdot \mathrm{min} / \mathrm{ml}) & 1.01 \pm 0.12 & 0.77 \pm 0.02 \\ \text { QS (ms) } & 16.5 \pm 2.1 & 13.2 \pm 1.0 \\ \mathrm{PEP}(\mathrm{ms}) & 42 \pm 1 & 34 \pm 1 \\ \mathrm{PEP} / \mathrm{LWET} & 0.59 \pm 0.02 & 0.43 \pm 0.02 \\ \mathrm{SW} \text { (ml.mmHg) } & 46 \pm 6 & 59 \pm 5\end{array}$

Abbreviations: dF/dt: aortic Elow acceleration; PF: peak flow; SV: stroke volume; Co: cardiac output; HR: heart rate; CVP: central venous pressure; PEP: pre-ejection periad; LVET: left ventricular ejection time: sh; stroke work. 


\section{Ejection fraction}

Ejection fraction was measured in $21 / 22$ months old SHR and WKY. SHR had a significantly reduced ejection fraction $(47 \pm 4 \% ; n=6)$ compared to agematched WKY $(70 \pm 2 \% ; \mathrm{n}=6)$.

\subsection{Discussion}

In man, besides myocardial infarction, hypertension is one of the major causes for congestive heart failure. After a stage in which compensatory mechanisms preserve cardiac performance in the face of a sustained increased work load, a stage of de-adaptation and subsequent heart failure occurs (Meerson, 1983). SHR provide a model for longitudinal studies of the natural progression of cardiac and circulatory alterations produced by chronic pressure overioad.

The purpose of this study was to investigate whether old SHR provide a model to study heart failure. Therefore, hemodynamic studies were performed in order to study heart failure on the basis of a depression of the cardiac function curve. However, in old SHR not only chronic hypertension but also the aging process itself can contribute to an altered cardiac performance (Walsh, 1987). Moreover, hypertension is suggested to be an accelerated aging process (Tomanek et al, 1981, 1982; Walsh, 1987; Van cle Laarse et al, 1987; Lakatta, 1987). Hemodynamic effects of aging in SHR were therefore compared to effects of aging in normotensive WKY. A variety of parameters were measured to study possible changes in underlying mechanisms.

At the age of 3 months, no differences were found between SHR and WKY, except for MAP, indicating no strain-related differences of cardiac function and other hemodynamic parameters. Left ventricular hypertrophy at this age (Pfeffer et al, 1976; Tomanek et al, 1981, 1982) functionally compensates the increased afterload in SHR, as indicated by a better cardiac performance compared to WKY at high afterload (Friberg et al, 1985).

From 3 to 17 months, both SHR and WKY show a slight reduction of HR with a slight increase of SV resulting in maintenance of baseline $\mathrm{CO}$. No differences were found between SHR and WKY for these parameters, nor for CVP. SHR remain hypertensive. In order to study cardiac performance, hearts were stimulated by volume loading. Neither differences related to aging nor to chronic hypertension were found for peak CO and peak SV. Similarly, Pfeffer et al (1976) did not find a decreased peak CO and SV after volume loading in open-chest, anesthetized 19 months old SHR compared to agematched WKY. In contrast, in a later study (Pfeffer et al, 1979b) 18 months female and male SHR showed a decreased peak SV compared to normotensive rats. The different results of these studies were probably due to different control groups (WKY in one study, WKY as well as Wistar rats in the other) rather than to differences in cardiac performance of SHR. Thus, data indicate that heart failure cannot be demonstrated as a depressed cardiac performance in conscious old SHR compared to age-matched WKY.

Although absolute values for peak CO and SV were not altered in old SHR, depression becomes overt if these values are presented per left ventricular weight (Pfeffer et al, 1976). Because left ventricular weight increased disproportionally to 
body weight despite stabilized MAP, this indicates changes in cellular mechanisms, rather than adaptation to the hemodynamic stimulus.

The cardiac hypertrophy in young SHR has been suggested to be necessary to maintain cardiac performance with increasing afterload (Noresson et al, 1979a). However, during the subsequent periods of increased left ventricular growth compared to normotensive rats, MAP remains constant. Whereas during growth to 12 months fractional volume percentage of myocytes increases and fractional volume percentage of interstitial tissue decreases, this trend reverses during growth from 12-18 months (Engelman et al, 1987). Since in the latter phase, myocyte diameter does not decrease, this implies dilution of myocytes in an increased tissue mass of interstitial material (Engelman et al, 1987). The increased interstitial tissue mass can be attributed to increased collagen (Pfeffer et al, 1979c; Weber et al, 1987). Unbalanced growth is furthermore demonstrated by capillary growth lagging behind that of left ventricular growth. Although Tomanek et al $(1981,1982)$ reported transient normalization at 15 months, a significant reduction of capillary density was found at 12 and 18 months (Engelman et al, 1987) indicating an increased maximal oxygen diffusion clistance.

In addition to the changes at tissue level, also intracellular changes occur. Volume percentage of mitochondria in myocytes decreases whereas volume percentage of myofibrils increases, resulting in a steep reduction of mitochondria/myofibril ratio (Tomanek et al, 1981). Furthermore, myosin isoenzyme pattern changes to a higher proportion of V3 type fibers with reduced myosin ATPase activity and contractility, but preserved maximally developed force (Vogt et al, 1987a; Winegrad et al, 1987). This transformation to a slower muscle is considered to be an adjustment leading to energy conservation (Vogt et al, 1987a).

Ventricular growth from 3-18 months can thus be attributed to an increase of interstitial tissue rather than to myocyte hypertrophy. The changes in extracellular matrix not only increase myocardial stiffness (Mirsky et $\mathrm{all}_{1}$, 1983) but also reduce oxygen supply to the myocytes because of increased diffusion distance. Mitochondrial changes suggest lower ATP production and reduced energy supply to myofibrils. Furthermore, a pressure overload induced shift to V3 type fibers is associated with decreased contractility and reduced calcium re-uptake in the sarcoplasmic reticulum indicating reduced relaxation (Scheuer et al, 1987). These changes would result in a large, stiff, slowly contracting and relaxing heart which is in accordance with the results in the present study. In addition, an increased left ventricular cavity to wall ratio as reported by Mirsky et al (1983) is associated with increased systolic wall stress (Vogt et al, 1987b). This structural dilatation is inversely related to liber shortening velocity and ejection fraction (Vogt et al, 1987a).

Significantly altered PEP, PEP/LVET and SW, while cardiac performance is preserved, indicate that the former parameters are the most sensitive markers for cardiac dysfunction in the present study. Prolongation of PEP could be attributed to a decreased conductance and/or decreased rate of left ventricular pressure rise, $\mathrm{dP} / \mathrm{dt}$ (Craige, 1980). Also increased MAP could prolong PEP (Weissler, 1968). Although the shorter QS and lower MAP in WKY could contribute to the difference in PEP between old SHR and WKY, it cannot explain the difference in PEP between old and young SHR, because QS and MAP are similar in these rats. HR dependency of PEP, discussed in the literature (Weissler, 1968; Lewis et al, 1977; Spodick et al, 1984; Rousson et al, 1987) was not found in the present study. Thus, increased PEP in the present study probably reflects impaired contractility. 
In contrast, LVET is usually found to be inversely related to HR (Craige, 1980; Rousson et al, 1987). Since rats of the same age and strain represent samples from the same population, the relationship between HR and LVET were analyzed per group. For all groups, this relationship was highly significant. LVET at HR $=0$, LVETI, was calculated according to Weissler (1968), using the regression equation of each group. Results indicate that not only increased PEP but also reduced LVETI contribute to the increased PEP/LVETI in old SHR. The ratio PEP/LVET is inversely related to ejection fraction in man (Garrard et al, 1970; Spodick et al, 1987). Corrected for HR changes, effects of PEP/LVETI are even more pronounced than uncorrected values. Data, thus, indicate a reduced ejection fraction, which is further corroborated by the ejection fractions as actually measured at 22 months of age. Because SV is maintained this suggests left ventricular dilatation. Similarly, left ventricular dilatation and reduced ejection fraction were found in 18 months SHR by Pfeffer et al (1979b) with increased left ventricular end-diastolic pressure and normal right atrial pressure. However, elevated work is needed to maintain SV. Thus, in the present study in 17 months old SHR, cardiac performance was maintained but indicators of mechanical properties of the heart were altered, suggesting impaired contractility, reduced ejection fraction from a dilated left ventricle, and increased work for the same SV. Similarly, Mirsky et al (1983) and Vogt et al (1987b) claimed impaired contractility and ventricular dilatation to be early determinants of the evolution of left ventricular dysfunction when cardiac performance was not yet impaired.

The above would suggest that, when SHR grow older, heart failure becomes overt. Indeed, Pfeffer et al (1979c) reported decreased baseline SV and decreased peak SV and $\mathrm{CO}$ per left ventricular weight in 21 months old male SHR. When absolute values for peak SV and CO were analyzed, effects remain. Mirsky et al (1983) also showed reduced baseline and peak $\mathrm{Cl}$ in 24 months old female SHR. Both studies reported further dilatation and reduced ejection fraction. Structural changes like increased volume percentage of interstitial tissue and decreased volume percentage of myocytes can be explained by increasing cell death and fibrotic scarring (Engelman et al, 1987) and also indicate impaired cardiac performance.

However, results in the present study show an increase in baseline and peak SV and $\mathrm{CO}$ in 20 months old survivors. PEP values indicate comparable contractility to 17 months old SHR while increased PEP/LVET suggest a further reduction of ejection fraction. Actually measured ejection fractions confirm this observation. Although methods are completely different, similar results for ejection fractions were found in 18 months old male SHR compared to normotensive controls (Pfeffer et al, 1979b) and in 24 months but not in 18 months old female SHR compared to age-matched WKY (Mirsky et al, 1983). Reduced ejection fraction is a strong indicator for cardiac failure.

Hemodynamic measurements in the 20 months old SHR were obtained from a highly selected group of old animals. Since mortality was very high, only rats with the best physical condition and, probably, the best cardiac performance would have survived and been measured, resulting in a high mean cardiac performance. Assuming that cardiac output at 17 months has a normal distribution and that the $5 \%$ of 17 months old SHR with the best cardiac performance would have survived, and have been studied at the age of 20 months, then $\mathrm{CO}$ could be estimated from the mean at 17 months plus twice the standard deviation. The result, $121 \mathrm{ml} / \mathrm{min}$, is comparable to the $\mathrm{CO}$ found at 20 months $(125 \mathrm{ml} / \mathrm{min})$. 
However, the highest baseline $\mathrm{CO}$ found in 17 months old SHR was still 10 $\mathrm{ml} / \mathrm{min}$ lower than the mean for 20 months SHR, indicating that this high cardiac performance may also represent a subsequent stage in the disease. Progressive volume overload due to impaired renal function, as indicated by increased recovery time, on top of the chronic pressure overload to the left ventricle, might be an explanation for these results. This suggestion, however, implies that this strain of SHR may die because of high output failure rather than of low output failure.

Nevertheless, reduced ejection fraction, increased recovery time and excessive pleural effusate clearly indicate cardiac failure. Since these phenomena do not differentiate between hemodynamically compensated low output failure and high output failure, interpretation of the results remains troublesome.

In conclusion, in 17 months old SHR heart failure could not be demonstrated as a depressed cardiac function curve, although indicators of mechanical properties of the heart suggest evolution of left ventricular dysfunction. Increased mortality after 17 months of age in SHR hampers interpretation of data in older SHR, but in these older rats the cardiac function curve was not depressed either. Since the purpose of this study was to evaluate whether the old SHR would be a suitable model to study heart failure, and in particular low output failure, the conclusion would be negative, since pathophysiological studies of the final stage of the disease are complicated by increased mortality. In addition, although reduced ejection fraction indicates failure, development of high output failure cannot be excluded. 



\section{THE CORONARY ARTERY LIGATED RAT AS A MODEL FOR HEART FAILURE}

\subsection{Introduction}

Myocardial infarction is one of the major causes of congestive heart failure in man. Therefore, animal models for heart failure have been cleveloped on the basis of experimental myocardial infarction or ischemia. Whereas ligation of the left descending coronary artery in rats has been a widely used technique for induction of cardiac arrhythmias (Curtis et al, 1987), there is growing interest for the use of this model to study the pathophysiology and therapy of heart failure. However, hemodynamic effects of coronary artery ligation in rats, presented in the literature are conflicting (Pfeffer et al, 1979a; Botting et al, 1983, Johnston et al, 1983; Drexler et al, 1986a; Chan et al, 1987). Discrepancies could be explained by the use of (different) anesthetics, acute surgery and of measurement at different times after ligation.

The aim of the present study was to evaluate whether the model of coronary artery ligation in rats may be suited for hemodynamic studies of the pathophysiology and therapy of heart failure. Therefore, hemodynamic changes consequent to the loss of contractile myocardium were investigated without interference of effects of acute surgery or anesthesia. Studies were performed in conscious rats, chronically instrumented for hemodynamic measurements. Effects on baseline hemodynamics and cardiac performance after volume loading were studied at different limes following coronary artery ligation. Acute effects were studied immediately after ligation up to $24 \mathrm{hr}$ later. Three and 5 weeks after ligation represent stages al which healing processes are considered to be complete (Fishbein et al, 1978; Pfeffer et all, 1979a) and damaged myocardiam is replaced by scar tissue. Chronic effects of the loss of vital myocardium may be represented by the effects at 3 months after ligation.

\subsection{Experimental protocols}

Experiments were performed in male Wistar rats, weighing $250-350$ grams at the start of the protocol. Hemodynamics were measured at five stages post myocardial infarction: 5 min, 24 hr, 3 weeks, 5 weeks and 3 months. The aim of the study was to inwestigate hemodynamic changes consequent to the loss of contractile myocardium 
without interfering effects of acute surgery or anesthesia. Therefore, slightly different protocols were used at $5 \mathrm{~min}$ and $24 \mathrm{hr}$ following infarction than for the other groups. This resulted in an acute and a long-term protocol.

\subsubsection{Acute effects of coronary artery ligation}

\section{Surgery protocol}

Surgery was performed in two stages, which greatly improved survival. In the first stage, rats were submitted to both left and right thoracotomy, in order to prepare the animals for coronary artery ligation at a later occasion and for measurement of aortic blood flow as described in chapter 2. Animals were allowed to recover at least 6 days from this preliminary surgery. The surgery was done aseptically and no antibiotics were given. Survival of this surgery was over $95 \%$. Following the recovery period, animals were re-anesthetized with ether and catheters for measurements of CVP and MAP, and for infusions were implanted (cf. section 2.4). Electrodes for ECG registration were also placed during this surgery stage. Animals were allowed another 2 days for recovery.

\section{Measuring protocol}

The total protocol for measurements encompassed 4 days. On the first day, animals were connected to the measuring equipment and signals were monitored on a Grass model 7D polygraph. After equilibration for 45-60 min, resting values for all variables were obtained during $15 \mathrm{~min}$. Then, $12 \mathrm{ml}$ of warm $\left(37^{\circ} \mathrm{C}\right)$ Ringer's solution was infused in $\mathbb{1}$ min. During this intervention, all hemodynamics were monitored continuously. The volume load increased $\mathrm{CO}$ to its plateau level after about $45 \mathrm{~s}$ in spite of further increasing CVP. Therefore, CO over the last $10 \mathrm{~s}$ of volume loading was regarded as the maximally stimulated $\mathrm{CO}$. Following the infusion, registration was continued during another $30 \mathrm{~min}$. Within this period, CVP had returned to baseline values. Two days later, animals were reconnected to the equipment and again allowed to stabilize for 45-60 min. After the stabilization period, they received an i.v. injection of $2 \mathrm{mg} / \mathrm{kg}$ lidocaine to prevent complications by arrhythmias that might occur during ischemia. In some rats, this injection caused a transient small reduction of MAP which was normalized within 2 min. After 2-3 min, the coronary artery ligature was pulled cautiously until typical changes indicative of ischemia (ST elevation) occurred in the ECG. In sham animals, the ligature was pulled comparably. At this point, the metal obturator was again introduced into the PE-100 catheter containing the ligature. This fixed the ligature so that it could not slip loose. Hemodynamics stabilized within $2-3$ min and were monitored continuously during the first 5-10 min following coronary occlusion. Twenty-four hr later, animals were again connected to the equipment, baseline values were obtained and the volume infusion protocol described above was repeated. After the last measurements, rats were killed and the size of infarction was determined as described in chapter 2 . 


\subsubsection{Long-term effects of coronary artery ligation}

\section{Surgery protocol}

Rats were subjected to coronary artery ligation under pentobarbital anesthesia as described in chapter 2. Infarcts were allowed to heal. One week before hemodynamic measurements rats were anesthetized with pentobarbital and an electromagnetic flow probe was implanted on the ascending aorta (cf. section 2.4.1). Rats were allowed to recover 5-6 days from this surgery. Following the recovery period, animals were reanesthetized with ether and catheters for MAP and CVP measurements and infusions, and ECG electrodes (cf. section 2.4.2-2.4.5) were implanted. Experiments started at least one day after this surgery.

\section{Measuring protocol}

Hemodynamic measurements were performed at 3 or 5 weeks or 3 months after myocardial infarction. Rats were connected to the measuring equipment (electromagnetic flowmeter, pressure transducers and ECG amplifier) and signals were fed into the "real-time data processing system" as described in section 2.5. Data were stored on a hard disk for later analysis.

After equilibration for $45-60 \mathrm{~min}$, baseline values for all variables were obtained during 15 min. Then, a volume load was given, while hemodynamics were monitored continuously (cf. section 2). Registration was continued till CVP had returned to its preinfusion value or maximally for 60 min. After finishing the experiments, rats were killed and infarct sizes were determined as described in section 2.3. Sham rats were measured only at 5 weeks and 3 months post myocardial infarction.

\subsection{Results}

\subsubsection{Coronary artery ligation}

\section{Mortality}

From 25 rats submitted to the coronary artery ligation in the conscious animal, 8 rats had no infarction. From the remaining 17 rats, 5 rats $(29 \%)$ died between 5 min and $24 \mathrm{hr}$ after tightening the ligature. Hemodynamics before and $5 \mathrm{~min}$ after ligation of these rats are presented in table 4.1. Profound reductions of blood pressure and cardiac output rather than ventricular fibrillation were the cause of death as could be seen from ECG registration. These 5 rats were excluded from further analysis.

No behavioral responses were noticed when ligation was performed in the conscious rat.

For coronary artery ligation performed under pentobarbital anesthesia, mortality was determined for a group of 107 rats. From this group $29 \%$ of the rats died within $48 \mathrm{hr}$ after ligation, whereas another $4 \%$ died between $48 \mathrm{hr}$ and 3 weeks. No mortality was found between 3 and 5 weeks after infarction, except for 1 rat that died during surgery. However, $68 \%$ of the rats, that survived the first 5 weeks, died within 
Table 4.1 Hemodynamic characteristics of the 5 animals that died within $24 \mathrm{hr}$ after coromary artery ligation (CAL)

Before CAL

$110 \pm 4$

$3.6 \pm 1 \cdot 3$

$375 \pm 10$

$89 \pm 3$

$237 \pm 7$

$1.20 \pm 0.08$

$25 \pm 1$
5 min after CAL

$60+9$

$\operatorname{CVP}\left(\mathrm{cm} \mathrm{H}_{2} \mathrm{O}\right)$
HR $\left.(\min )^{-1}\right)^{-1}$
$\operatorname{Co}\left(\mathrm{ml} \cdot \mathrm{min}^{-1}\right)$
$\mathrm{SV}(\mu \mathrm{l})$

TPR (mmHg. m $\left.1 n \cdot m 1^{-1}\right)$

$\mathrm{SW}(\mathrm{mmHg} \cdot \mathrm{ml})$
$9.2 \pm 3.3$

$324 \pm 38$

$40 \pm 10$

$132 \pm 27$

$1.54 \pm 0.64$

$7 \pm 3$

Table 4.2 Characteristics of experimental groups at different points in time after coronary artery ligation

$24 \mathrm{hr} \quad 3$ weeks $\quad 5$ weeks 3 months

\begin{tabular}{|c|c|c|c|c|c|}
\hline $\mathbf{n}$ & $\begin{array}{l}\text { sham } \\
\text { Infarct }\end{array}$ & $\begin{array}{r}8 \\
12\end{array}$ & $\begin{array}{r}0 \\
12\end{array}$ & $\begin{array}{r}7 \\
12\end{array}$ & $\begin{array}{l}6 \\
6\end{array}$ \\
\hline $\mathrm{BW}(\mathrm{g})$ & $\begin{array}{l}\text { sham } \\
\text { infarct }\end{array}$ & $\begin{array}{l}308+9 \\
299 \pm 5\end{array}$ & $316 \pm 9$ & $\begin{array}{l}395 \pm 16 \\
334 \pm 10\end{array}$ & $\begin{array}{l}403 \pm 18 \\
388 \pm 37\end{array}$ \\
\hline $\mathrm{HW}(\mathrm{g})$ & $\begin{array}{l}\text { sham } \\
\text { infarct }\end{array}$ & $\begin{array}{l}- \\
-\end{array}$ & $1.00 \pm 0.05$ & $\begin{array}{l}1.35 \pm 0.16 \\
1.18 \pm 0.09\end{array}$ & $\begin{array}{l}1.43 \pm 0.19 \\
1.34 \pm 0.17\end{array}$ \\
\hline $\mathrm{HW} / \mathrm{BW}(\mathrm{g} / \mathrm{kg})$ & $\begin{array}{l}\text { sham } \\
\text { infarct }\end{array}$ & - & $3 . \overline{1 \pm 0.2}$ & $\begin{array}{l}3.4 \pm 0.4 \\
3.5 \pm 0.3\end{array}$ & $\begin{array}{l}3.5 \pm 0.5 \\
3.4 \pm 0.4\end{array}$ \\
\hline $\operatorname{Inf}$, size $(\%)$ & $\begin{array}{l}\text { shant } \\
\text { infarct }\end{array}$ & $\begin{array}{c}0 \\
28 \pm 5\end{array}$ & $33 \pm 4$ & $\begin{array}{c}0 \\
31 \pm 3\end{array}$ & $\begin{array}{c}0 \\
41 \pm 4\end{array}$ \\
\hline Resp. $\left(\min n^{-1}\right)$ & $\begin{array}{l}\text { sham } \\
\text { infarct }\end{array}$ & - & $139_{ \pm}^{-} 14$ & $\begin{array}{l}112 \pm 10 \\
147 \pm 11\end{array}$ & $\begin{array}{l}104 \pm 5 \\
145 \pm 8\end{array}$ \\
\hline LVEC (mm) & $\begin{array}{l}\text { sham } \\
\text { infarct }\end{array}$ & - & $15.8 \pm 1.0$ & $\begin{array}{c}7.8 \pm 0.6 \\
15.6 \pm 1.4\end{array}$ & $\begin{array}{c}9.5 \pm 0.6 \\
21.9 \pm 3.0 *\end{array}$ \\
\hline
\end{tabular}

Abbreviations: BH: body weight; HW: heart weight; inf. size: infarct size; resp.: respiration rate; LVEC: mean left ventricular endocardial circumference. *:significantly different from sham control.

Hemodynamics before and at $5 \mathrm{~min}$ and $24 \mathrm{hr}$ after ligation were measured in the same animals. Characteristics of these experimental groups are given at 24 hr postligation. Effects at 3 and 5 weeks and 3 months were measured in separate groups of rats. 
the following 7 weeks. Some of these rats had severe peripheral oedema. Because of the high mortality the experiment was terminated after obtaining data from 6 infarcted rats. At gross autopsy at the end of the experiments, most of the infarcted rats showed signs of congestion in liver and lungs and some of them had pleural effusion.

\section{Infarcts}

Infarct sizes were determined after hemodynamic measurements except for rats that died within $24 \mathrm{hr}$. Ligation of the left descending coronary artery resulted in an infarction in the free wall of the left ventricle. The septum was never involved. Infarcts were transmural except for the ones smaller than $10 \%$. For the majority of larger infarcts papillary muscles were not involved. Infarct sizes ranged from $4-58 \%$. In hearts with one day old infarcts, at gross inspection, neither dilatation of the left ventricle, nor wail thinning of the infarcted part had occurred. At 3 weeks after infarction, left ventricular wall at the site of infarction was replaced by sear tissue which was thinner than at comparable places in sham ventricles. Five weeks after ligation, mean endocardial circumferences of infarcted left ventricles were significantly increased, and comparable to those in 3 weeks infarcted rats. Three months after ligation endocardial circumference of infarcted left ventricles had further increased indicating progressive left ventricular dilatation from one day to 3 months after infarction (table 4.2). However, although not significantly, infarct sizes were higher in rats with 3 months old infarcts.

Table 4.3 Indicators of mechanical properties at different times following ligation

\begin{tabular}{|c|c|c|c|c|}
\hline & & 3 weeks & 5 weeks & 3 months \\
\hline п & $\begin{array}{l}\text { sham } \\
\text { infarct }\end{array}$ & $\overline{12}$ & $\begin{array}{r}7 \\
12\end{array}$ & $\begin{array}{l}6 \\
6\end{array}$ \\
\hline $\operatorname{PEP}$ (ans) & $\begin{array}{l}\text { shan } \\
\text { infarct: }\end{array}$ & $39 \pm 2$ & $\begin{array}{l}36 \pm 1 \\
39 \pm 1 *\end{array}$ & $\begin{array}{l}37 \pm 1 \\
41 \pm 3\end{array}$ \\
\hline PEP/LVET & $\begin{array}{l}\text { sham } \\
\text { infarct }\end{array}$ & $0.49 \pm 0.02$ & $\begin{array}{l}0.50 \pm 0.01 \\
0.53 \pm 0.01 *\end{array}$ & $\begin{array}{l}0.50 \pm 0.01 \\
0.52 \pm 0.04\end{array}$ \\
\hline $\mathrm{SW}(\mathrm{mmHg} \cdot \mathrm{ml})$ & $\begin{array}{l}\text { sham } \\
\text { infarct }\end{array}$ & $20 \pm$ & $\begin{array}{l}25 \pm 1 \\
20 \pm 1\end{array}$ & $\begin{array}{l}26 \pm 1 \\
22 \pm 2 *\end{array}$ \\
\hline
\end{tabular}

Abbreviations: PEP: pre-ejection period;

PEP/LVET: PEP ratio to left ven-tricular ejection time; SW: stroke work.

* significantly different from sham rats 

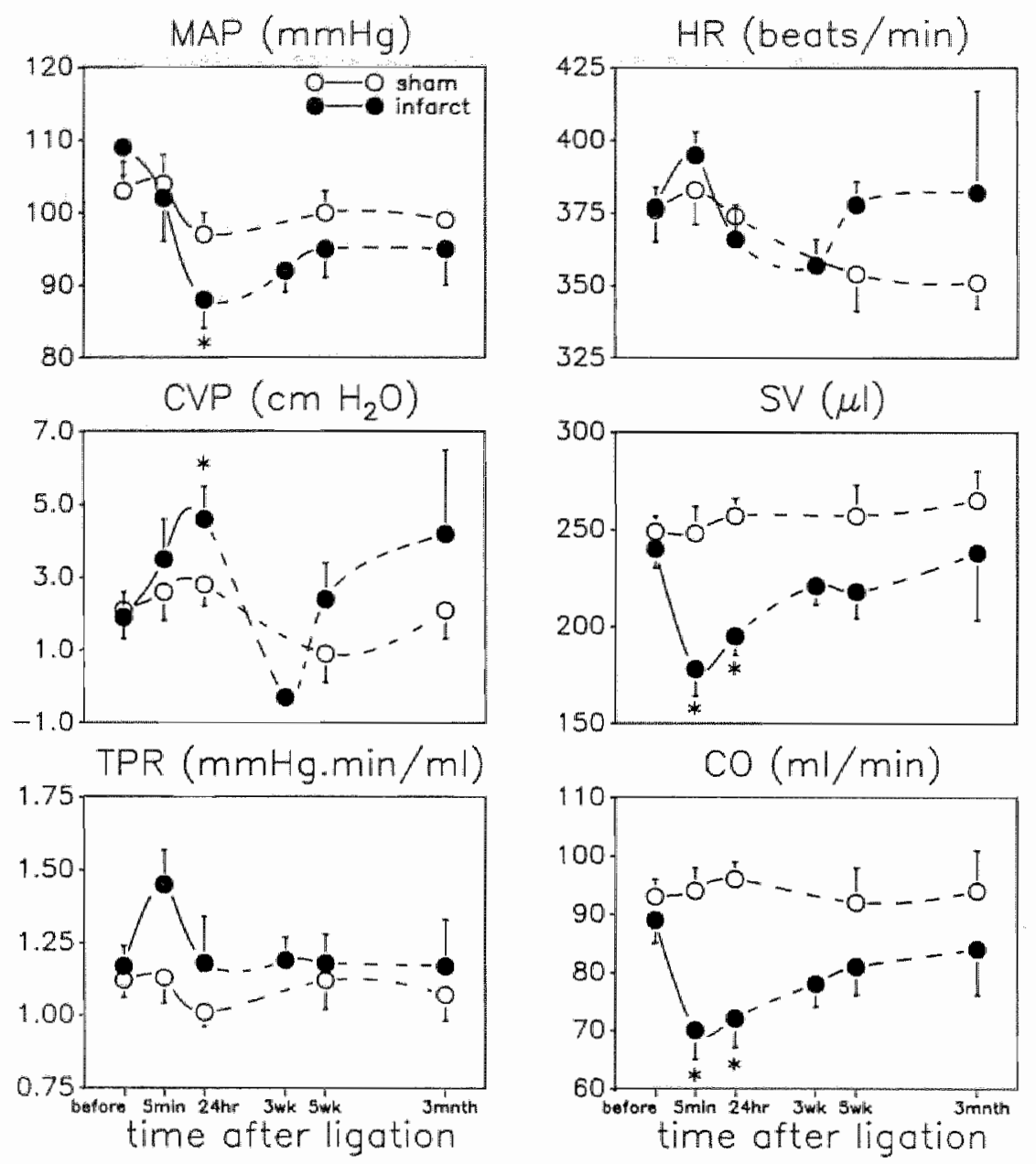

Fig. 4.1 Baseline hemodynamics before and 5 min, $24 \mathrm{hr}, 3$ weeks, 5 weeks and 3 nonth after ligation in sham and infarcted rats (mean $\pm S E M, n=6-12$ )

\subsubsection{Effects of coronary artery ligation}

\section{Experimental groups}

Characteristics of the experimental groups are summarized in table 4.2 Twenty four hr alter ligation left ventricular weight was significantly increased from $0.75 \pm 0.02$ to $0.84 \pm 0.02 \mathrm{~g}$. Decreased dry/wet weight ratio determined in 3 sham rats $(11.5 \pm 0.1 \%)$ and 3 infarcted rats $(9.9 \pm 0.1 \%)$ suggests myocardial oedema to 
contribute to the increased ventricular weight. Since body weight did not change, left ventricular weight related to body weight also increased significantly $(2.28 \pm 0.02$ and $2.84 \pm 0.08 \mathrm{~g} / \mathrm{kg}$ respectively).

At 3 weeks, no sham group was studied. Therefore no comparison could be made to sham rats. Five weeks after ligation, infarcted rats had a significantly lower body weight and heart weight compared to sham rats. Heart weight related to body weight, however, did not differ. Respiration rate was increased in post-infarcted rats at 5 weeks and 3 months.

\section{Effects of coronary artery ligation on baseline hemodynamics}

Effects of coronary artery ligation on baseline hemodynamics at different times following لigation are summarized in fig. 4.1. Before ligation, groups were not significantly different in any respect. Immediately (5 min) after ligation $\mathrm{CO}$ was significantly reduced in direct relation to infarct size. A significantly lowered SV accounted for this, because HR was even slightly increased. Since MAP and CVP were not changed significantly at this moment, and $\mathrm{CO}$ was decreased, TPR was increased. This increase, however, did not reach significance. One day after ligation, CO was still depressed while MAP was significantly decreased and CVP was significantly increased. HR and TPR were normalized. SW was significantly decreased at $5 \mathrm{~min}$ and at $24 \mathrm{hr}$ after ligation (sham: $25 \pm 2$ and $24 \pm 1 \mathrm{mmHg} \cdot \mathrm{ml}$, infarct: $20 \pm 2$ and $18 \pm 2 \mathrm{mmHg} \cdot \mathrm{ml}$ at $5 \mathrm{~min}$ and $24 \mathrm{hr}$ respectively). These results indicate a state of acute heart failure.

Three weeks after infarction, values for MAP, HR, TPR and CO were normalized or tended to normalize. In rats with 5 weeks old infarcts none of these parameters was significantly different from sham rats, indicating hemodynamic compensation. Only indicators of mechanical properties of the heart (PEP, PEP/LVET and SW) were significantly altered at 5 weeks, whereas PEP/LVET was not yet increased at 3 weeks (cf. table 4.3). PEP and SW (fig. 4.2) were related to infarct size. Three months after ligation, a tendency towards higher CVP, PEP and HR could be seen, but changes were not found to be significantly different from sham animals.
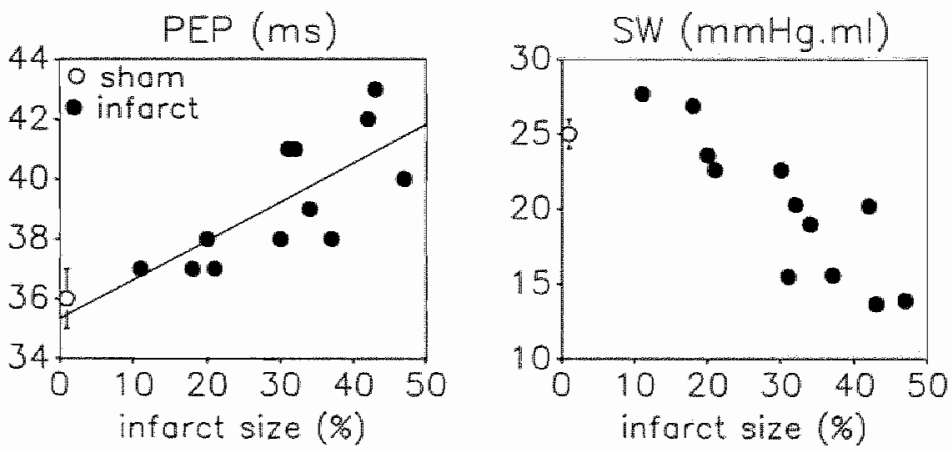

Fig. 4.2 Baseline PEP and $S W$ related to infarct size. The symbol at infarct size $0 \%$ represent mean $\pm S E M$ for sham rats 
Table 4.4 Hemodynamics after stimulation by volume loading at different times following ligation

\begin{tabular}{|c|c|c|c|c|c|}
\hline & & $24 \mathrm{hr}$ & 3 weeks & 5weeks & 3 months \\
\hline $\mathrm{n}$ & $\begin{array}{l}\text { sham } \\
\text { infarct }\end{array}$ & $\begin{array}{r}8 \\
12\end{array}$ & $\begin{array}{r}0 \\
12\end{array}$ & $\begin{array}{r}7 \\
12\end{array}$ & $\begin{array}{l}6 \\
6\end{array}$ \\
\hline $\mathrm{dF} / \mathrm{dt}\left(\mathrm{mil} / \mathrm{s}^{2}\right)$ & $\begin{array}{l}\text { sham } \\
\text { infarct }\end{array}$ & - & $489 \pm 27$ & $\begin{array}{l}543 \pm 17 \\
466 \pm 33 *\end{array}$ & $\begin{array}{l}575 \pm 40 \\
483 \pm 60\end{array}$ \\
\hline $\mathrm{PF}(\mathrm{ml} / \mathrm{min})$ & $\begin{array}{l}\text { sham } \\
\text { infarct }\end{array}$ & - & $393 \pm 22$ & $\begin{array}{l}451 \pm 16 \\
341 \pm 36 *\end{array}$ & $\begin{array}{l}471 \pm 25 \\
407 \pm 45\end{array}$ \\
\hline $\mathrm{co}(\mathrm{ml} / \mathrm{min})$ & $\begin{array}{l}\text { sham } \\
\text { infarct }\end{array}$ & $\begin{array}{c}134 \pm 3 \\
93 \pm 7\end{array}$ & $12 \overline{-}$ & $\begin{array}{l}152 \pm 5 \\
121 \pm 8 *\end{array}$ & $\begin{array}{c}156 \pm 6 \\
124 \pm 13 *\end{array}$ \\
\hline$S W(\mu \perp)$ & $\begin{array}{l}\text { sham } \\
\text { infarct }\end{array}$ & $\begin{array}{l}343 \pm 11 \\
247 \pm 16 *\end{array}$ & $301 \pm 16$ & $\begin{array}{l}388 \pm 15 \\
295 \pm 20\end{array}$ & $\begin{array}{l}400+9 \\
304 \pm 38\end{array}$ \\
\hline HR (beats/min) & $\begin{array}{l}\text { sham } \\
\text { infarct }\end{array}$ & $\begin{array}{l}392 \pm 15 \\
374 \pm 11\end{array}$ & $\frac{-}{401 \pm 5}$ & $\begin{array}{l}395 \pm 8 \\
413 \pm 10\end{array}$ & $\begin{array}{l}391+9 \\
422 \pm 40\end{array}$ \\
\hline MAP (momHg) & $\begin{array}{l}\text { sham } \\
\text { infarct }\end{array}$ & $\begin{array}{l}99 \pm 3 \\
85 \pm 7 *\end{array}$ & $8 \overline{-}+4$ & $\begin{array}{r}100 \pm 3 \\
94 \pm 4\end{array}$ & $\begin{array}{l}99 \pm 3 \\
95 \pm 5\end{array}$ \\
\hline $\operatorname{cVP}\left(\mathrm{cm} \mathrm{H}_{2} 0\right)$ & $\begin{array}{l}\text { sham } \\
\text { infarct }\end{array}$ & $\begin{array}{l}14.0 \pm 1.2 \\
17.4 \pm 1.1 *\end{array}$ & $14 . \overline{6} \pm 0.9$ & $\begin{array}{l}11.0 \pm 1.1 \\
15.1 \pm 1.0 *\end{array}$ & $\begin{array}{l}11.8 \pm 1.2 \\
15.6 \pm 2.7\end{array}$ \\
\hline PEP (ns) & $\begin{array}{l}\text { sharn } \\
\text { infarct }\end{array}$ & - & $35 \pm 2$ & $\begin{array}{l}33 \pm 1 \\
37 \pm 1 *\end{array}$ & $\begin{array}{l}33 \pm 1 \\
39 \pm 4\end{array}$ \\
\hline PER/LWET & $\begin{array}{l}\text { sham } \\
\text { infaret }\end{array}$ & - & $0.43 \pm 0.02$ & $\begin{array}{l}0.40 \pm 0.01 \\
0.47 \pm 0.02 *\end{array}$ & $\begin{array}{l}0.41 \pm 0.02 \\
0.52 \pm 0.11\end{array}$ \\
\hline SW (mollg.m & $\begin{array}{l}\text { sham } \\
\text { infaret }\end{array}$ & $\begin{array}{l}30 \pm 2 \\
21 \pm 3 *\end{array}$ & $23 \pm 2$ & $\begin{array}{l}34 \pm 1 \\
25 \pm 2 *\end{array}$ & $\begin{array}{l}35 \pm 2 \\
26 \pm 4 *\end{array}$ \\
\hline
\end{tabular}

Abbreviations: see section 2.5

*:significantly different from sham rats

Effects of coronary artery ligation on stimulated hemodynamics

Cardiac function curves were made by stimulating the heart through the Frank-Starling mechanism. This curve relates cardiac output to central venous pressure as a measure of filling pressure. Peak CO was determined 2 days before ligation and 24 hr, 3 weeks, 5 weeks and 3 months after ligation. The groups used to measure acute effects of ligation were not different in any respect before ligation. Data are summarized in table 4.4. 


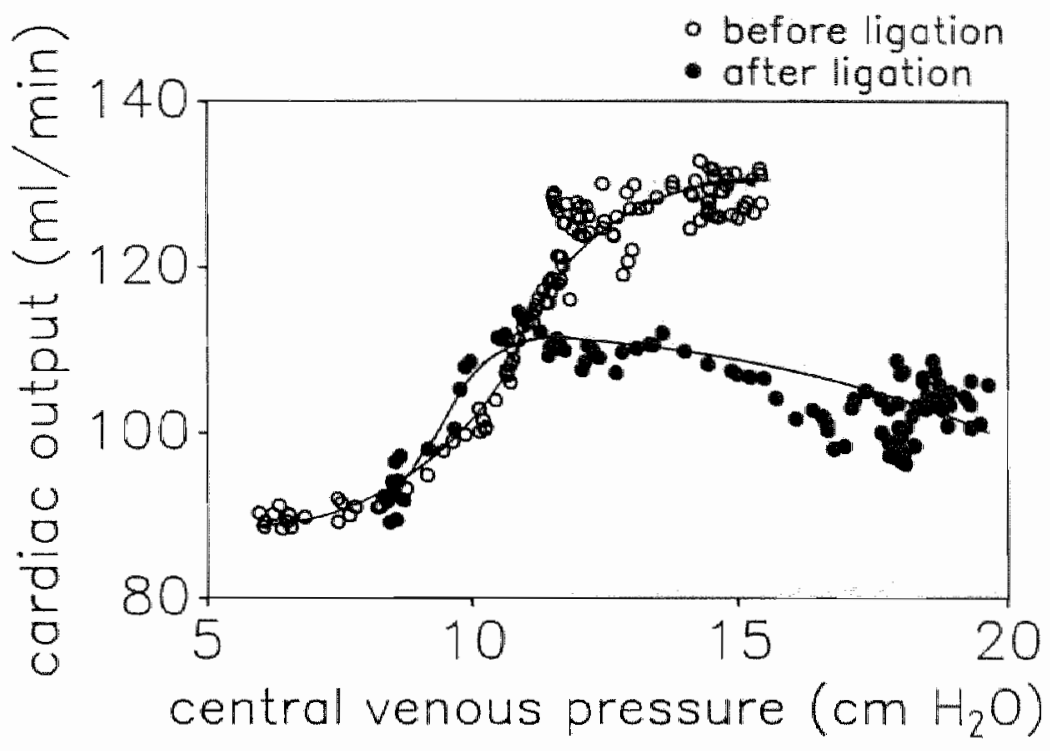

Fig. 4.3 Cardiac function curves before and after coronary artery ligation in the same rat (infarct size 22\%)

The acute effect of ligation on the cardiac function curve is illustrated in fig. 4.3. presenting this curve before and after coronary artery ligation in the same rat. After ligation, baseline CVP was increased. Stimulated CO was depressed, while CVP rose further. Mean effects are summarized in fig. 4.4. Twenty-four hr after ligation, mean values for peak $\mathrm{CO}, \mathrm{SV}$ and $\mathrm{SW}$ were significantly depressed (cf. table 4.4) in direct relation to infarct size.

Whereas at 5 weeks after ligation, baseline $\mathrm{CO}$ was only slightly decreased, stimulated $\mathrm{CO}$ was depressed significantly. Indeed, after volume loading, $\mathrm{dF} / \mathrm{dt}, \mathrm{PF}$, SV and SW were significantly depressed, too. However, infarcts up to $21 \%$ did not affect these parameters, suggesting full hemodynamic compensation of these infarcts. In fact, stimulated cardiac output plotted against infarct size at $24 \mathrm{hr}, 3$ and 5 weeks after ligation (fig. 4.5) suggests progression of this process. Whereas at $24 \mathrm{hr}$ even small infarcts decreased $\mathrm{CO}$, at 5 weeks infarcts had to be larger than $21 \%$ to cause measurable hemodynamic effects. Three months after ligation even the performance of hearts with $31 \%$ infarction can reach sham values after volume loading. 


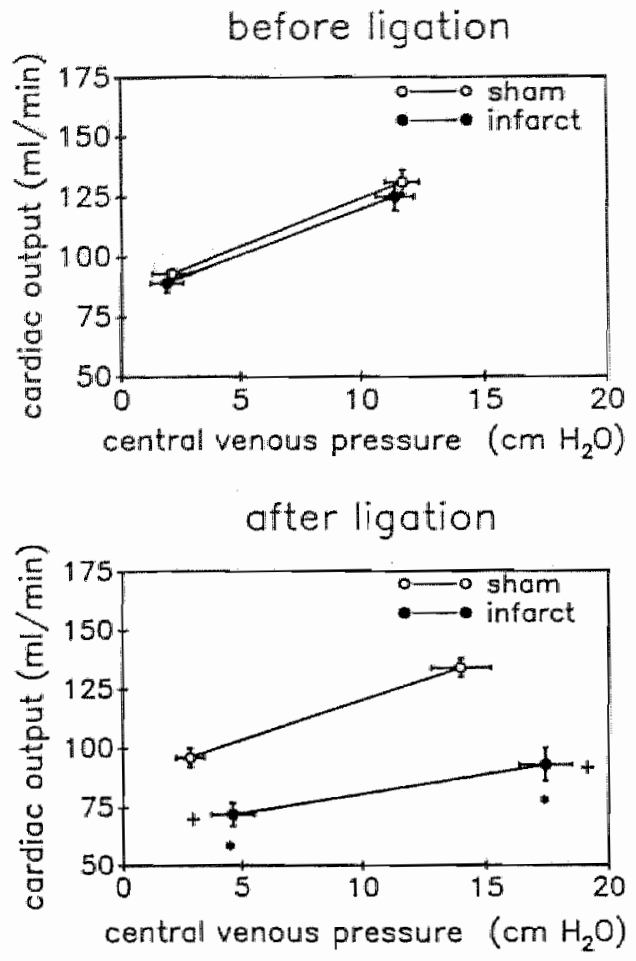

Fig. 4.4 Cardiac function curves before and after coronary artery ligation (mean $\pm S E M$, $n=9-11) ;+$ significant differences in CVP; *:significant differences in $C O$

24 hours

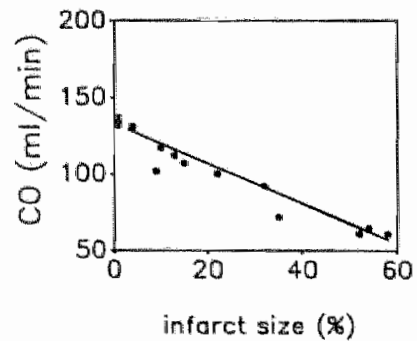

3 weeks

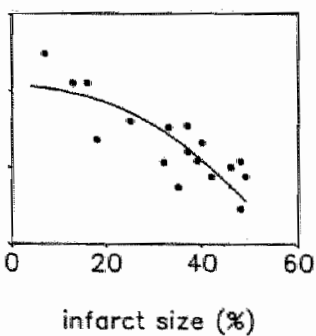

5 weeks

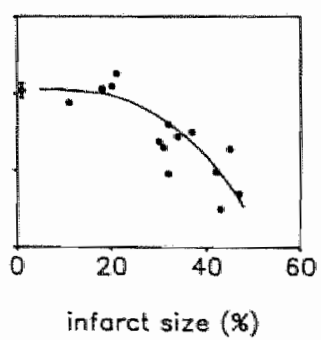

Fig. 4.5 Stimulated cardiac output related to infarct size at $24 \mathrm{hr}, 3$ weeks and 5 weeks after ligation. The open symbol at infarct size 0\% represent mean $\pm S E M$ for sham rats 


\subsection{Discussion}

The purpose of this study was to perform a hemodynamic evaluation whether the model of conscious rats with myocardial infarction is a suited model for studies on pathophysiology and therapy of heart failure. Ligation of the left descending coronary artery in rats is widely used as an experimental approach to the induction of ventricular arrhythmias and congestive heart failure (reviewed by Curtis et al, 1987). In the present study, this technique resulted in exclusively left ventricular infarcts which were, except the ones smaller than $10 \%$, transmural. The interventricular septum was never involved and in most of the infarcted ventricles papillary muscles were spared. This is in accordance with the findings of Spadaro et al (1980). Severe arrhythmias at the time of occlusion were prevented by prophylactic lidocaine administration and arrhythmias at the time of hemodynamic measurements were rare. Thus, left ventricular infarcts in the present study cause loss of contractile myocardium without concomitant complications of arrhythmias or mitral valve insufficiency.

Acute mortality in the present study was relatively low compared to $40-60 \%$ reported in the literature (Pfeffer et al, 1979a; Drexler et al, 1986b). The prophylactic administration of lidocaine may explain this, since lidocaine caused a profound increase of survival after infarction in conscious rats (Johnston et al, 1983). The increased mortality after 5 weeks survival could be due to severe pulmonary and/or peripheral congestion because severad. rats were oedematous.

\subsubsection{Acute effects of infarction}

The immediate result of the loss of contractile myocardium is a decrease of stroke volume and cardiac output in direct relation to the amount of damaged left ventricular tissue. At this time, MAP was maintained but 24 hr later, MAP was decreased whereas CVP was increased. Since CO was still depressed, this situation was indicative of acute cardiac failure. Also in man, reduced SV and CO are common phenomena following acute myocardial infarction (Kupper et al, 1977). The extent to which hemodynamic changes like tachycardia, increased CVP and arterial hypotension occur in man has been related to infarct size (Alonso et al, 1983; Handler, 1985).

In animals, the acute effects of coronary artery ligation have been reported to be highly variable, depending upon absence or presence, as well as the type of anes. thesia and the time after surgery when hemodynamics were measured. In halothane anesthetized rats, CI and SI were depressed at 2 days following ligation but not at day one. No effects were found on MAP, HR and CVP (Peres, 1987). In conscious rats, CO was not found to be decreased within 3 days (Ratz et al, 1980; Drexler et al, 1986a) suggesting no cardiac depression immediately following infarction.

However, in the previously mentioned studies (Ratz et al, 1980, Drexler et al, 1986a; Peres, 1987), infarcted rats were compared to sham operated rats within 3 days after surgery for coronary artery ligation. The surgery itself is reported to be cardiodepressive even in sham rats (Van de Wouw et al, 1987) which could mask the effects of myocardial infarction. Curtis et al (1987) stated that in order to study effects of ligation in conscious rats, thoracic surgery should be performed 6-10 days 
beforehand. If so, then MAP decreased acutely while HR was not affected or slightly decreased (Botting et al, 1983; Johnston et al, 1983). In the present study, rats were previously prepared for coronary artery ligation, but only rats which survived the whole protocol were used for analysis. If the data of rats that died within $24 \mathrm{hr}$ (cf. table 4.1), were pooled with the dlata of survivors, then acute coronary artery ligation resulted in decreased MAP, CO, SV and SW, increased CVP and unchanged HR.

In the present study early mortality may be due to lowered blood pressure and CO rather than arrhytimias. Since at this time after ligation infarcts cannot be detected, it is not known whether this was due to very large infarcts or insufficient hemodynamic compensation. Reduced MAP and increased mortality due to blockade of sympathetic reflexes after acute ligation (Botting et al, 1983) indicate the importance of early sympathetic activation for survival. Contribution of cardiac afferent nerve stimulation to this activation (Peres, 1987) strongly depends on recovery time after thoracic surgery and anesthetic effects (Pagani et al, 1985).

The decrease of MAP in survivors $24 \mathrm{hr}$ after ligation was associated with a return of initially increased TPR to control values and a persistent reduction of $\mathrm{CO}$. This pattern of response is consistent with an initially increased but later waning sympathetic nerve activity. In dogs, plasma catecholamine levels have been observed to increase within one min after ligation (Karlsberg et al, 1979). In rats $2 \mathrm{hr}$ after ligation, noradrenaline content of the left ventricle was decreased due to an increased noradrenaline turnover (Bernauer, 1983; Inove et al, 1987). A similar pattern of sympathetic activation and increased noradrenaline turnover is found in man acutely following infarction (Schömig, 1988). The fact that sympathetic reflexes could be influenced by anesthesia or recovery time after surgery (Cox et al, 1979; Pagani et al, 1985) may explain some of the conflicting observations mentioned earlier.

In conclusion, acutely after coronary artery ligation, heart failure is evident from a depressed cardiac function, increased CVP and decreased MAP while HR was not affected.

\subsubsection{Long-term efrects of infarction}

After acute myocardial infarction, repair mechanisms are activated to replace the irreversibly damaged myocardium by scar tissue. In rats this process is considered to be complete 3 weeks after infarction (Pfeffer et al, 1979a; Fishbein et al, 1981). In addition, mechanisms are activated to functionally compensate the loss of contractile myocardium and act to maintain systemic perfusion. Whereas acutely after infarction, responses may be predominantly of neural origin, at later stages humoral and structural changes may become more important. Several cardiovascularly active hormones may have been elevated within days following ligation. After the repair period (first 3 weeks following ligation) plasma atrial natriuretic peptide (ANP) was found to be significantly increased (Tikkanen et al, 1987; Hodsman et al, 1988a), whereas plasma renin activity, and levels of aldosterone and arginine vasopressin are comparable to those in sham operated rats (Hodsman et al, 1988b). In accordance with this, an increased ANP at $24 \mathrm{hr}$ after ligation, and an increased ANP but not plasma renin concentration at 5 weeks after ligation (data not shown) were observed in the present model. There is no evidence for sodium retention despite the decreased renal blood flow (Hostetter et al, 1983; Brenner et al, 1984; Drexler et al, 1987a) since plasma osmolality, plasma $\mathrm{Na}^{+}$and total exchangeable body sodium are not different from 
sham rats (Hodsman et al, 2988b; Hostetter et al, 1983). The increased ANP could functionally compensate for the reduced renal blood flow. Howewer, renal responses to ANP may be impaired by a decreased number of intrarenal binding sites (Tsunoda et al, 1988).

Structural changes occur not only in the damaged part of the myocardium (i.e. scar formation) but also in the spared myocardium. Ventricular hypertrophy, prefer entially in the right ventricle as a response to an increased left ventricular end-diastolic pressure, was reported already 3 days after ligation (Anversa et al, 1985a). Total left ventricular weight was not decreased despite thinning of the infarcted part, indicating also hypertrophy of the spared left ventricle (Pfeffer et al, 1979a; Anversa et al, 1985a, 1986). This hypertrophy results in an increased force of the spared myocardium. However, increased myocyte size implies operation at a lower energy supply since capillary growth is lagging behind and mitochondria/myofibril ratio is reduced (Anversa et al, 1986). Sarcolemma surface area, containing ion-transport systems important for contraction, is decreased in relation to cell contents (Meerson, 1983). In addition, lack of concomitant sympathetic innervation (Meerson, 1983) and a shift in myosin isoenzyme pattern towards more V3 fibres with lower ATP-ase activity (Geenen et al, 1989) suggests lower contractility and impaired response to sympathetic stimulation.

All these responses could have contributed to the hemodynamic situation at $3-5$ weeks compared to $24 \mathrm{hr}$ after ligation in the present study. Whereas at $24 \mathrm{hr}$ after ligation, $\mathrm{CO}$ was depressed with increased CVP, these changes were no longer evident at 3 and 5 weeks after infarction. In fact, at baseline condition, only indicators of mechanical properties of the heart (PEP, PEP/LVET and SW) were altered 5 weeks after infarction.

Prolongation of the PEP could be attributed to a delayed conductance and/or a decreased rate of left ventricular pressure rise (dP/dt) (Craige, 1980) . Since QS interval was not increased and also $H R$ and MAP had not changed, the increase in PEP in the present study reflects an impaired contractility (Craige, 1980). The relationship between. PEP and infarct size supports this. Because PEP is the only parameter affected by infarets $<21 \%$, it may be the most sensitive marker for the loss of contractility.

The ratio PEP/LVET is used as an indicator of left ventricular ejection fraction; increased PEP/LVET correlates with a decreased ejection fraction (Craige, 1980; Rousson et al, 1987). Maintained baseline SV and concomitantly increased laft ventricular diastolic volume, as derived from endocardial circumferences in the present study, also indicate a decreased ejection fraction. The trend from 3 to 5 weeks suggests progression of this process, which is also indicated by results of Pfeffer et al (1982b).

Since baseline CVP was normalized, failure at this stage seems to be restricted to the left ventricle. Right ventricular hypertrophy and increased left ventricular end-diastolic pressure (LVEDP) (Pfeffer et al, 1979; Drexler et al, 1986a,b) in addition to signs of pulmonary congestion and oedema, and shortness of breath support this suggestion.

The transient nature of the reduction of baseline $\mathrm{CO}$ indicates partly compensation of the loss of contractile myocardium. In accordance with Drexler et al (1987a), no effects on baseline CI, SI, MAP, TPR or HR were found in conscious rats 42 days after infarction. However, if infarcts $<35 \%$ were excluded (Drexler et al, 1986b), decreased baseline CI, SI, SW and MAP were found with increased periphe- 
ral resistance. In anesthetized rats, baseline $\mathrm{CI}$ and MAP were depressed at large infarcts (>47\%), whereas HR was not affected (Pfeffer et al, 1979a). Taken together, in accordanice to the present study, this indicates compensation of cardiac function related to infarct size.

Thus, compensatory responses during the repair period result in normalization of most hemodynamic parameters (CVP, CO, SV, MAP, HR and TPR) whereas indicators of mechanical properties (PEP, PEP/LVET, SW) are still altered. After volume loading, however, heart failure was evident from a depressed cardiac function related to the infarct size. Small infarcts were hemodynamically completely compensated.

Results found 3 months after ligation should be interpreted carefully since mortality was strongly increased after 5 weeks. Mortality is related to the size of the infarct (Pfeffer et al, 1985b; Sweet et al, 1987), suggesting that survivors represent rats with smaller infarcts rather than rats with 3 months old infarcts. However, the measured infarct size in this group was slightly higher than in the group of rats with 5 weeks old infarcts. Since it was not posssible to measure the size of the same infarct at different points in time, it is not known whether the measured sizes at different times are comparable. Therefore, no selection has been made based upon infarct size.

Stimulated $\mathrm{CO}$ and SW were found to be significantly depressed whereas the latter parameter was also depressed at baseline. Trends could be seen indicating progression of heart failure from 5 weeks after ligation. A further left ventricular dilatation, also reported by Pfeffer et al (1982b), in addition to an increase of CVP, suggests further development of left ventricular failure accompanied by right ventricular failure. Severe peripheral oedema in rats that died earlier supports this. In survivors no effects of infarction on MAP or on HR were found. Comparable results were reported by Sweet et al (1987) and Pfeffer et al (1987). In the latter study, left ventricular end-diastolic pressure and volume were increased related to infarct size while ejection fraction was decreased.

Thus, also in survivors 3 months after infarction, heart failure is evident from a depressed cardiac function without changes in HR or MAP. A tendency may be seen for accompanying right ventricular failure at this stage.

In conclusion, in the present rat model, 3 stages can be distinguished: a stage of acute heart failure with high CVP and depressed CO, SV, SW and MAP, a stage of "compensated" heart failure with normalized CVP, CO, SV and MAP, but dilated left ventricle and altered mechanical properties, and a stage in which a trend to "decompensated" heart failure, viz. higher CVP and further dilatation of the left ventricle, becomes evident. In all 3 stages, heart failure could be demonstrated by a depressed cardiac function curve. Depression of cardiac performance, was related to the size of the infarction, which determines the severity of heart failure. Whereas in the acute stage, cardiac performance is decreased even for small infarcts, after the repair period these infarcts are hemodynamically compensated. Thus, this model provides an opportunity for longitudinal studies on the pathophysiology of heart failure, combined with a broad scala of severity of heart failure at different time points. Results from this chapter will serve as basis for the therapy studies in the next chapters. 


\section{MILRINONE IN HEART FAILURE}

\subsection{Introduction}

Therapy of heart failure includes the use of positive inotropic and vasodilating drugs. Milrinone is a phosphodiesterase inhibitor with positive inotropic and vasodilating properties in man. Whereas there is general agreement about hemodynamic improvement after acute administration (Baim et al, 1983; Sys et al, 1986; Piscione et al, 1987; Evans, 1989), literature about progression of the disease and mortality is conflicting (LeJemtel et al, 1986; Monrad et al, 1986; Baim et al, 1986) possibly caused by difference in patients, interference with additional therapy, or design of the studies. Recently a large long-term milrinone survival study has been announced (Packer, 1989). Results after 3 months of therapy, however, indicate increased mortality rather than reduction (DiBianco et al, 1989).

In infarcted rats, milrinone is also reported to have positive inotropic and vasodilating effects (Emmert et al, 1987; Drexler et al, 1987b). Moreover, milrinone not only improves hemodynamics after acute therapy, but also increases survival with chronic therapy in infarcted rats (Sweet et al, 1988). This suggests long-term hemodynamic improvement.

However, no detailed hemodynamic studies with prolonged milrinone are available in rats with heart failure. Therefore, in the present study hemodynamic effects of 2 weeks milrinone as monotherapy were investigated in conscious rats with heart failure after myocardial infarction. Effects were compared to hemodynamic changes after acute administration in normal healthy rats and in rats with heart failure. Because the purpose of the study was to investigate effects of milrinone in heart failure, hemodynamics were not measured before 3 weeks after infarction. At this time repair processes are considered to be complete (Fishbein et al, 1978; Pfeffer et al, 1979) and rats have heart failure. Two weeks therapy was given from 3 to 5 weeks post infarction since this represents a rather stable period in the development of the disease (chapter 4). 


\subsection{Experimental protocols}

Experiments were performed in male Wistar rats weighing $250-350 \mathrm{~g}$ at the start of the protocol. Hemodynamic effects of milrinone were studied in conscious rats in 3 experiments. Acute effects of the drug were investigated in normal healthy rats and in chronically infarcted rats. Results were compared to effects of two weeks therapy in chronically infarcted rats.

\subsubsection{Acute effects in normal healthy rats}

\section{Surgery protocol}

Rats were anesthetized with pentobarbital and an electromagnetic flow probe was implanted (cf. section 2.4.1). Rats were allowed to recover 5-6 days from this surgery. Following the recovery period animals were reanesthetized with ether and an arterial catheter for MAP measurement and 2 venous catheters for infusions were implanted (cf. section 2.4.2, 2.4.4). Experiments started at least one day after this surgery.

\section{Measuring protocol}

Hemodynamic measurements were performed every other day in order to prevent effects of a previous volume load or drug infusion. In random order, rats were subjected to control volume load or volume load during infusion of 10,30 or $100 \mu \mathrm{g} / \mathrm{kg}$.min milrinone.

After connection to the measuring equipment, hemodynamics were allowed to stabilize for $45-60 \mathrm{~min}$. Then, baseline values were obtained and either a control volume load was given (cf. 2.5.1) or infusion was started (total infused volume $<1$ $\mathrm{ml}$ ). During the infusion, when hemodynamics had stabilized or at least $30 \mathrm{~min}$ later, the heart was also maximally stimulated by a volume load. Hemodynamics were monitored contimuously during the experiment (Grass polygraph).

\subsubsection{Acute effects in chronically infarcted rats}

\section{Surgery protocol}

Rats were subjected to coronary artery ligation as described in section 2.2.1. Infarcts were allowed to heal. Acute effects of milrinone were studied 3 weeks after ligation. One week before hemodynamic measurements rats were anesthetized with pentobarbital and electromagnetic flow probes were implanted. After 5-6 days recovery catheters were implanted for MAP and CVP measurements and for infusions (cf. section 2.4.1-2.4.4). Experiments were started at least one day following this surgery.

\section{Measuring protocol}

Hemodynamic measurements were performed at days 1 and 3 of the protocol. Rats were subjected to control volume load and to volume load during $30 \mu \mathrm{g} / \mathrm{kg}$.min milrinone in random order. Animals were connected to the measuring equipment and 
signals were fed into the real-time data processing system (cf. section 2.5). After equilibration for $45-60 \mathrm{~min}$, baseline values were obtained. Then either a control volume load was given or infusion was started. After at least 30 min infusion, when hemodynamics had stabilized, a volume load was given. Hemodynamic registration was continued till CVP had returned to its pre-infusion value or during maximally 60 min. Data were stored on a hard disk for later analysis. After finishing the experiments, rats were killed and infarct sizes were determined (cf. section 2.3).

\subsubsection{Two weeks therapy in chronically infarcted rats}

\section{Surgery protocol}

Rats were subjected to coronary artery ligation and infarcts were allowed to heal. Three weeks later, rats were divided into 3 groups. One group received no treatment whereas the other groups were treated with milrinone at 2 different dosages. Under light ether anesthesia osmotic minipumps (Alzet 2001, Palo Alto, USA) filled with milrinone solution were implanted subcutaneously between the shoulder blades. Milrinone therapy was given at 30 or $150 \mu \mathrm{g} / \mathrm{kg}$. hr subcutaneously. After one week pumps were replaced by new ones and electromagnetic flow probes were implanted. Following recovery of $5-6$ days, catheters for MAP and CVP measurements and for infusions were implanted. In addition, ECG electrodes were implanted (cf. 2,4.5). Hemodynamic measurements were performed at least one day after this surgery.

\section{Measuring protocol}

Hemodynamic measurements were performed after 2 weeks therapy. Rats were connected to the measuring equipment and signals were fed into preprocessor and computer for on-line derivation of hemodynamic parameters. After equilibration for 45-60 min, baseline values were obtained. Then, the heart was stimulated by volume load. Hemodynamics were monitored continuously till CVP had returned to baseline values or maximally 60 min after wollume load. Data were stored on a hard disk for later analysis. When the experiment was finished, the rats were killed for deternina tion of infarct sizes (cf. 2.3).

\subsection{Resullts}

\subsubsection{Acute effects in normal healthy rats}

Hemodynamic effects of acute milrinone on MAP, HR, SV and $\mathrm{CO}$ are presen. ted in table 5.1. Milrinone dose-dependently reduced SV and increased HR without effects on baseline CO. MAP was dose-dependently decreased. Effects reached statistical significance at $100 \mu \mathrm{g} / \mathrm{kg}$.min milrinone. Stimulated $\mathrm{CO}$, was dose-depen. dently increased due to an increase of both SV (not significant) and HR. Results indicate that milrinone has positive inotropic and chronotropic and vasodilating effects which are dose related. The positive inotropic effects only become overt when the heart is stimulated. 
Table 5.1 Effects of acute milrinone in normal healthy rats $(n=6-9)$. Baseline (c) and volume laad $V L$ (c) present values for control volume load, whereas baseline (o), baseline (d) and volume load, $V L$ (d) present baseline values before, baseline values during and stimulated values during drug infusion respectively.

\begin{tabular}{|c|c|c|c|c|c|}
\hline Dose mill Inone & $\begin{array}{l}\text { Contro } \\
\text { Baseline } \\
\text { (c) }\end{array}$ & $\begin{array}{l}1 \\
\text { VL } \\
(c)\end{array}$ & $\begin{array}{l}\text { Drug } \\
\text { Baseline } \\
\text { (o) }\end{array}$ & $\begin{array}{l}\text { Infusion } \\
\text { Baseline } \\
\text { (d) }\end{array}$ & $\begin{array}{l}\text { VL } \\
\text { (d) }\end{array}$ \\
\hline \multicolumn{6}{|l|}{$10 \mu \mathrm{g} / \mathrm{kg}$, min } \\
\hline 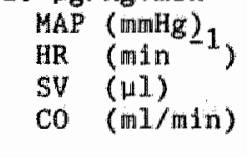 & $\begin{array}{l}108 \pm 4 \\
372 \pm 10 \\
268 \pm 12 \\
100 \pm 3\end{array}$ & $\begin{array}{l}109 \pm 4 \\
393 \pm 12 \\
405 \pm 9 \\
159 \pm 4\end{array}$ & $\begin{array}{l}109 \pm 1 \\
372 \pm 15 \\
270 \pm 17 \\
100 \pm 7\end{array}$ & $\begin{array}{c}105 \pm 2 \\
373 \pm 16 \\
270 \pm 16 \\
99 \pm 7\end{array}$ & $\begin{array}{l}107 \pm 3 \\
397 \pm 23 \\
393 \pm 17 \\
156 \pm 12\end{array}$ \\
\hline 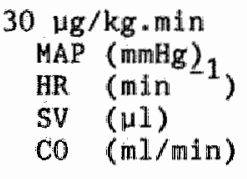 & $\begin{array}{l}113 \pm 6 \\
392 \pm 20 \\
254 \pm 20 \\
98 \pm 3\end{array}$ & $\begin{array}{l}116 \pm 5 \\
403 \pm 11 \\
361 \pm 13 \\
143 \pm 7\end{array}$ & $\begin{array}{l}105 \pm 5 \\
376 \pm 18 \\
251 \pm 21 \\
96 \pm 7\end{array}$ & $\begin{array}{l}94 \pm 6 \\
427 \pm 22 \\
240 \pm 27 \\
106 \pm 7\end{array}$ & $\begin{array}{r}89 \pm 5^{+} \\
422 \pm 12 \\
398 \pm 11 \\
166 \pm 7^{+}\end{array}$ \\
\hline 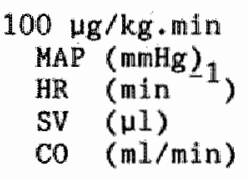 & $\begin{array}{l}109 \pm 3 \\
381 \pm 1,4 \\
262 \pm 14 \\
100 \pm 3\end{array}$ & $\begin{array}{l}110 \pm 3 \\
402 \pm 8 \\
405 \pm 12 \\
161 \pm 4\end{array}$ & $\begin{array}{l}106 \pm 3 \\
371 \pm 6 \\
273 \pm 11 \\
101 \pm 5\end{array}$ & $\begin{array}{l}76 \pm 2 * \\
461 \pm 5 * \\
232 \pm 11 * \\
108 \pm 7\end{array}$ & $\begin{array}{l}78 \pm 2^{*} \\
442 \pm 6^{+} \\
439 \pm 15 \\
196 \pm 6^{+}\end{array}$ \\
\hline
\end{tabular}

* significantly different from baseline before infusion (baseline $(0)$ )

significantly different from stimulated values (VL (c) of control volume load

Abbreviations: MAP: mean arterial pressure; HR: heart rate; SV: strake volume; CO: cardiac output

\subsubsection{Acute effects in chronically infarcted rats}

Since only infarcts of more than $20 \%$ had measurable hemodynamic consequences (cf. section 4.3.2 and 4.3.3) data of rats with smaller infarcts were excluded from this analysis. Effects of $30 \mu \mathrm{g} / \mathrm{kg}$.min milrinone in chronically infarcted rats are summarized in table 5.2. Chronic infarction caused a depression of baseline and stimulated SV and CO. However, since there was no sham group at 3 weeks this could not be statistically verified. Similar to normal healthy rats at the same dose of milrinone a slight tachycardia was found without effects on baseline $\mathrm{SV}, \mathrm{CO}$ and MAP, nor on baseline CVP $\left(2.3 \pm 0.6\right.$ and $1.4 \pm 0.9 \mathrm{~cm} \mathrm{H}_{2} \mathrm{O}$ before and during millrinone infusion respectively). $\mathrm{CO}$ after stimulation was significantly improved caused by $12 \%$ increase in SV and $6 \%$ increase in $\mathrm{HR}$, but was still below normal values in healthy rats $(143 \pm 7 \mathrm{ml} / \mathrm{min})$. 
Table 5.2 Effects of acute milrinone $(30 \mu \mathrm{g} / \mathrm{kg} . \mathrm{min})$ in chronically infarcted rats $(n=8)$

\begin{tabular}{|c|c|c|c|c|c|}
\hline & $\begin{array}{l}\text { Contr } \\
\text { Baseline } \\
\text { (c) }\end{array}$ & $\begin{array}{l}\mathrm{VI}_{x} \\
\text { (c) }\end{array}$ & $\begin{array}{l}\text { Drug } \\
\text { Baseline } \\
(0)\end{array}$ & $\begin{array}{l}\text { Infusion } \\
\text { Baseline } \\
\text { (d) }\end{array}$ & $\begin{array}{l}\text { VL } \\
\text { (d) }\end{array}$ \\
\hline MAP & $94 \pm 5$ & $89 \pm 5$ & $90 \pm 6$ & $87_{ \pm 5}$ & $81 \pm 7$ \\
\hline $\mathbb{H R}$ & $380 \pm 15$ & $401 \pm 6$ & $375 \pm 11$ & $414 \pm 16$ & $423 \pm 13$ \\
\hline sv & $186 \pm 14$ & $251_{ \pm} 17$ & $211 \pm 10$ & $208 \pm 14$ & $282 \pm 13$ \\
\hline co & $70 \pm 5$ & $1.00 \pm 6$ & $79 \pm 3$ & $85 \pm 4$ & $119 \pm 7^{4}$ \\
\hline
\end{tabular}

For explanation abbreviations and units, see table 5.1

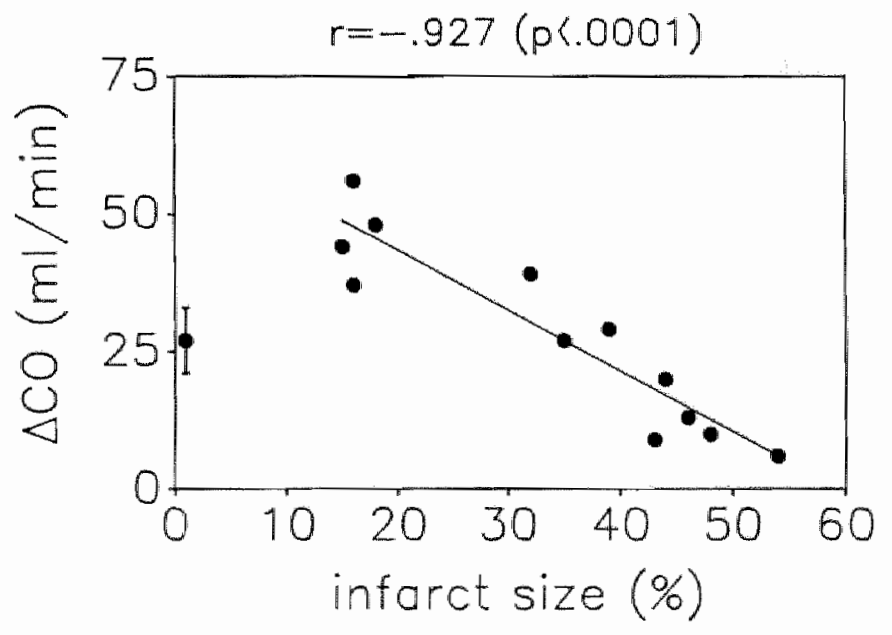

Fig. 5.1 Increased stimulated cardiac output related to infarct size $(n=12)$. The symbot at infarct size 0\% represents mean $\pm S E M$ for normal healthy rats. 
Responses may be related to the severity of heart failure. To test this hypothesis the relationship between improvement of stimulated $\mathrm{CO}$ after acute milrinone (VL(d)-VL(c); table 5.2) and infarct size was analyzed using linear regression. Improvement was found to be inversely related to infarct size $(r=-0.927, p<0.0001)$. For the analysis also small infarcts were included and responses of these hearts were even enhanced compared to normal hearts (symbol at infarct size 0\%; fig. 5.1). The inverse relationship was also found for the increase in $\mathrm{HR}(\mathrm{r}=-0.643)$ but not for the increase of SV $(r=-0.515)$.

In conclusion, if small infarcts were excluded, effects of acute milrinone in chronically infarcted rats in proportion to changed baseline values were comparable to the ones in normal healthy rats at the same dose. For the small infarcts, however, the inotropic effect of acute milrinone was increased rather than maintained. When all infarcts are included, the increase of stimulated $\mathrm{CO}$ was found to be inversely related to infarct size.

Table 5.3 Characterization of experimental groups

\begin{tabular}{|c|c|c|c|c|}
\hline & Sham & Infarct & $\begin{array}{l}\text { Milrinone } \\
(30 \mathrm{\mu g} / \mathrm{kg} \cdot \mathrm{hr})\end{array}$ & $\begin{array}{l}\text { Milrinone } \\
(150 \mathrm{\mu g} / \mathrm{kg} \cdot \mathrm{hr})\end{array}$ \\
\hline n & 7 & 8 & 8 & 7 \\
\hline inf. size $(\%)$ & 0 & $37 \pm 2^{+}$ & $36 \pm 2^{+}$ & $41 \pm 3^{*}$ \\
\hline $\mathrm{BW}(\mathrm{g})$ & $395+16$ & $326 \pm 13^{*}$ & $312 \pm 8^{*}$ & $327 \pm 11^{+}$ \\
\hline $\mathrm{HW}$ (mg) & $1352 \pm 163$ & $1284 \pm 137$ & $957 \pm 25^{+*}$ & $1128 \pm 43$ \\
\hline $\mathrm{HW} / \mathrm{BW}$ (mg/g) & $3.5 \pm 0.4$ & $3.8 \pm 0.4$ & $3.1 \pm 0.1$ & $3.5 \pm 0.2$ \\
\hline Resp. $\left(\right.$ min $\left.n^{-1}\right)$ & $112 \pm 10$ & $147 \pm 11^{+}$ & $135 \pm 13$ & $145 \pm 13$ \\
\hline LVEC (mm) & $7.8 \pm 0.6$ & $15.6 \pm 1.4^{+}$ & $15.9 \pm 0.7^{+}$ & $15 \cdot 2 \pm 1.3^{+}$ \\
\hline
\end{tabular}

Abbreviations: inf. size: infarets size; BW: body weight; HW: heart weight; resp.: respiration frequency; LVEC: mean left ventricular endocardial circumference

* significantly different from non-treated infarcted rats

slignificantly different from sham operated rats

\subsubsection{Efrects of two weeks administration of milrinone in chronically infarcted rats}

\section{General}

In non-treated infarcted rats only one rat died during the "treatment" period. For the low and high dose, 6 and 3 rats died, respectively. All these rats, except one, died during surgery for probe implantation. After exclusion of data from rats with infarcts 
Table 5.4 Baseline hemodynamics after 2 weeks milrinone compared to non-treated infarcted rats and sham rats

\begin{tabular}{|c|c|c|c|c|}
\hline & Sham & Infarct & $\begin{array}{l}\text { Milrinone } \\
30 \mathrm{\mu g} / \mathrm{kg} \cdot \mathrm{hr}\end{array}$ & $\begin{array}{l}\text { Milrinone } \\
150 \mathrm{\mu g} / \mathrm{kg} \cdot \mathrm{hr}\end{array}$ \\
\hline $\mathrm{n}$ & 7 & 8 & 8 & 7 \\
\hline $\mathrm{dF} / \mathrm{dt}(\mathrm{ml} / \mathrm{s})$ & $421 \pm 23$ & $339 \pm 30$ & $440 \pm 21^{*}$ & $471 \pm 31^{*}$ \\
\hline $\mathbb{P F}(\mathrm{m} 1 / \mathrm{min})$ & $366 \pm 19$ & $285 \pm 24^{4}$ & $350 \pm 15$ & $382 \pm 24^{*}$ \\
\hline $\mathrm{SV}(\mu \mathrm{l})$ & $257 \pm 16$ & $199 \pm 17^{+}$ & $223 \pm 11$ & $252 \pm 13 *$ \\
\hline $\operatorname{CVP}\left(\mathrm{cm} \mathrm{H} \mathrm{H}_{2}{ }^{0}\right)$ & $0.9 \pm 0.8$ & $1.8 \pm 1.5$ & $0.8 \pm 0.4$ & $1.2 \pm 0.5$ \\
\hline $\operatorname{TPR}(\mathrm{mmHg} \cdot \mathrm{min} / \mathrm{ml})$ & $1.12 \pm 0.10$ & $1.25 \pm 0.15$ & $1.13 \pm 0.04$ & $1.04 \pm 0.06$ \\
\hline $\mathrm{SW}(\mathrm{mmHg} \cdot \mathrm{ml})$ & $25 \pm 1$ & $17_{ \pm 1}+$ & $21 \pm 1$ & $24 \pm 2 *$ \\
\hline PEP (ms) & $36 \pm 1$ & $40 \pm 1^{+}$ & $37 \pm 2$ & $34 \pm 2^{\star}$ \\
\hline PEP/LVET & $0.49 \pm 0.01$ & $0.54 \pm 0.02^{+}$ & $0.51 \pm 0.02$ & $0.48 \pm 0.03$ \\
\hline$Q S$ (ms) & $16.7 \pm 0.9$ & $17.0 \pm 1.9$ & $11.2 \pm 0.8^{\star}$ & $14.0 \pm 1.1$ \\
\hline
\end{tabular}

Abbreviations: $\mathrm{dF} / \mathrm{dt}$ : positive aortic flow acceleration; PF: peak flow; SV: stroke volume; CVP: central venous pressure; TPR: total peripheral resistance; SW: stroke work; PEP: pre-ejection period PEP/LVET: ratio PEP and left ventricular ejection time: QS: duration of QS complex of $\mathbb{E C G}$.

+ Significant effects of infarct compared to sham

* Significant effects of therapy compared to non-treated infarcts

of sizes $<21 \%$, groups had comparable infarct sizes. Groups are characterized in table 5.3. All infarcted groups had significantly lower body weights compared to sham rats, but no differences were found due to therapy. Although the group treated with the low dose had a significantly reduced heart weight, the ratio HW/BW was not significantly altered. No effects of milrinone therapy were found on the increased respiration rate, nor on left ventricular dilatation (LVEC).

\section{Hemodynamic effects}

Hemodynamic effects of 5 weeks infarction are described in detail in chapter 4 . When small infarcts were excluded, depression of cardiac performance was significant, even at baseline conditions (table 5.4).

After stimulation by volume loading effects of infarction became more pronounced: depressed $\mathrm{dF} / \mathrm{dt}, \mathrm{PF}, \mathrm{SV}, \mathrm{SW}$ and $\mathrm{CO}$, while PEP and PEP/LVET were increased. MAP and HR were not altered (table 5.5). 

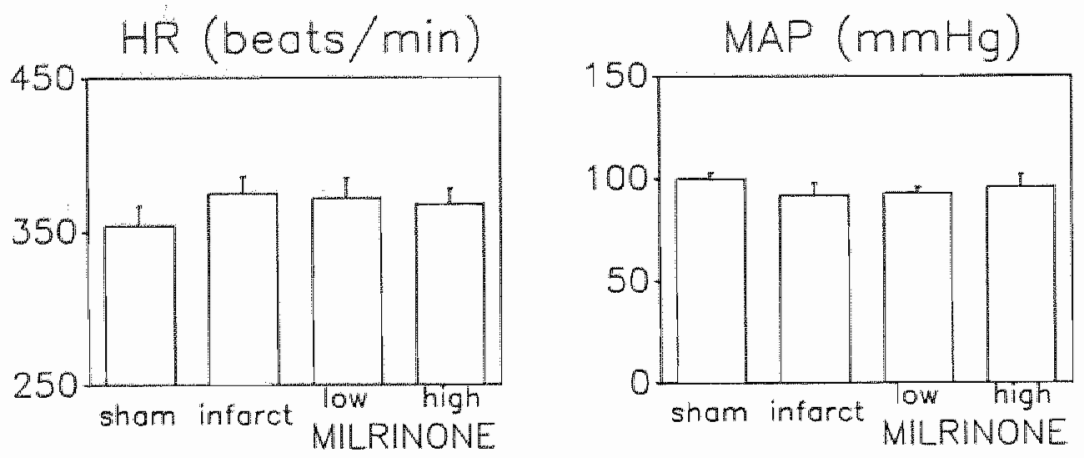

Fig. 5.2 Effects after 2 weeks of milrinone therapy on baseline heart rate (HR) and mean arterial pressure (MAP) compared to sham and non-treated infarcted control rats.

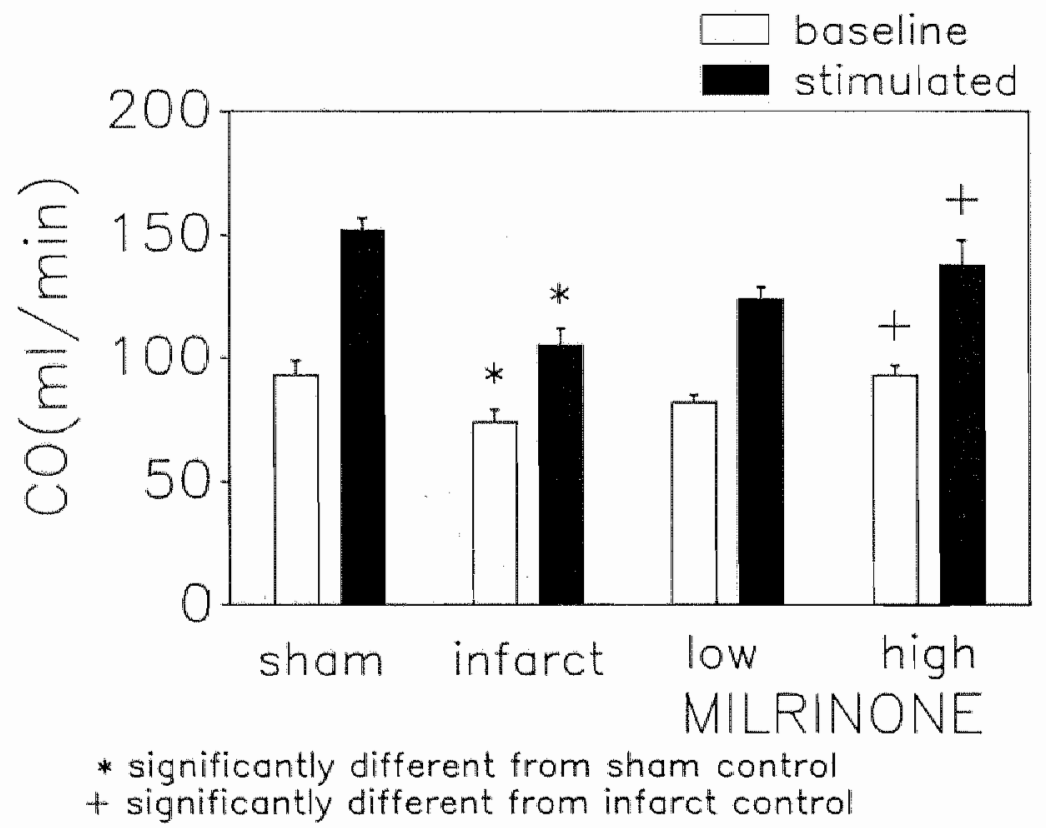

Fig. 5.3 Effects of 2 weeks of milrinone therapy on baseline and stimulated cardiac output $(\mathrm{CO}) ;$ mean $\pm \mathrm{SEM}$. 
Table 5.5 Effects of two weeks milrinone therapy on hemodynamics after stimulation by volume load

\begin{tabular}{|c|c|c|c|c|}
\hline & Sham & Infarct & $\begin{array}{l}\text { Milrinone } \\
30 \mathrm{\mu g} / \mathrm{kg} \cdot \mathrm{hr}\end{array}$ & $\begin{array}{l}\text { Milrinone } \\
150 \mathrm{\mu g} / \mathrm{kg} \cdot \mathrm{hr}\end{array}$ \\
\hline$n$ & 7 & 8 & 8 & 7 \\
\hline $\mathrm{dF} / \mathrm{dt}$ & $543 \pm 17$ & $406 \pm 30^{*}$ & $531 \pm 26$ & $557 \pm 56 *$ \\
\hline $\mathrm{PF}$ & $451 \pm 16$ & $348 \pm 19^{+}$ & $422 \pm 17$ & $453 \pm 37 *$ \\
\hline sV & $388 \pm 15$ & $256 \pm 17$ & $312 \pm 11$ & $346 \pm 21 *$ \\
\hline $\mathrm{HR}$ & $395 \pm 9$ & $412_{ \pm} 14$ & $394 \pm 9$ & $397 \pm 11$ \\
\hline SW & $34 \pm 1$ & $20 \pm 2^{+}$ & $25 \pm 1$ & $29+3 k$ \\
\hline PEP & $33 \pm 1$ & $38 \pm 2^{+}$ & $32 \pm 2$ & $29 \pm 2 *$ \\
\hline PEP/LVET & $0.40 \pm 0.01$ & $0.48 \pm 0.02^{+}$ & $0.40 \pm 0.02 *$ & $0.37 \pm 0.03 *$ \\
\hline
\end{tabular}

Two weeks of milrinone treatment affected neither MAP nor HR (fig. 5.2), but dose-dependently restored the depressed cardiac function (fig. 5.3). The improvement was altogether reflected in SV since HR did not change. In fact, all hemodynamic changes due to infarction were restored dose-dependently (table 5.4 and 5.5) by milrinone. Improvement was statistically significant at the high dose of milrinone both for changes at baseline and after volume load (except for baseline PEP/LVET).

Thus, whereas acute administration of milrinone improves cardiac performance by only $19 \%$ due to a slight increase in both HR and SV, 2 weeks of millrinone treatment restored cardiac performance by $38 \%$ which was completely due to an increased SV. The long-term effect was achieved by rates of administration which presumably have no acute inotropic effects $(2-10 \%$ of the minimum effective rate for acute inotropic effects).

\subsection{Discussion}

Milrinone is a potent inhibitor of phosphodiesterase III (Earl et al, 1986; Silver, 1989). Inhibition of this enzyme results in an increased intracellular cAMP (Earl et al, 1986). Elevation of CAMP in cardiac muscle leads to an increased force of contraction and an increased rate of relaxation, whereas in vascular smooth muscle it produces relaxation (Sys et al, 1987; Evans, 1989).

The purpose of this study was to investigate hemodynamic effects of acute and 2 weeks milrinone therapy in rats with heart failure. Howewer, before inwestigating this, acute hemodynamic effects were studied in normal healthy rats to verify the positive inotropic and vasodilating effects of milrinone in conscious rats. 


\subsubsection{Acute effects of milrinone in normal healthy rats}

Milrinone dose-dependently increased HR and decreased SV which resulted in maintained baseline $C O$. The lack of effect on cardiac output may be explained on the basis of the vasodilating effects of milrinone. Milrinone has been shown to cause venodilation (Defeo et al, 1985; Colucci, 1989), resulting in pooling of blood in capacitance vessels and, hence, reducing filling pressures. When in the present experiment, the heart was loaded by rapid volume infusion (cardiac function curve), the maximally reached $\mathrm{CO}$ was dose-dependently increased by milrinone. The increase was not only due to tachycardia but also to an increased $\mathrm{SV}$, supporting the hypothesis that reduced filling pressure accounts for the lack of increase in $\mathrm{CO}$ at baseline conditions. Thus, in normal healthy rats, milrinone dose-dependently induces vasodilation whereas positive inotropic effects of milrinone, possibly másked al baseline by reduced filling pressures, become overt after stimulation by volume loading.

\subsubsection{Milrinone in rats with heart failure}

After exclusion of small infarcts because of hemodynamic compensation (cf. chapter 4), acute effects of milrinone in chronically infarcted rats are comparable to those in normal healthy rats. Although absolute effects were smaller in infarcted rats, changes were proportionally similar. Nevertheless, stimulated $C O$ was still depressed as compared to normal values in healthy rats. Baseline $\mathrm{SV}$ and $\mathrm{CO}$ were reduced due to infarction and were not affected by acute milrinone, despite a reported increase in $\mathrm{dP} / \mathrm{dt}$ in anesthetized infarcted rats (Emmert et al, 1987). Again, a reduction of left ventricular filling pressure could mask inotropic effects on $\mathrm{SV}$ and $\mathrm{CO}$. In another study in conscious infarcted rats, milrinone at a dose of $3 \mu \mathrm{g} / \mathrm{kg}$.min caused a significant increase in CI with no effect on MAP and a slight (5\%) but significant increase in HR (Drexler et al, 1987b). In the present study, when the heart was stimulated by increasing filling pressure, maximally achieved $\mathrm{CO}$ was increased by millninote if the increase of stimulated $\mathrm{CO}$ represents the positive inotropic effect, then the inotropic effect is found to be inversely related to the size of infarction, i.e., to the severity of heart failure (chapter 4 ). This relationship could be attributed 10 the percentage of spared myocardium avalable for positive inotropic stimulation. However, since the inverse relationship was also found for the HR response, which is not directly related to the amount of contractile myocardium, but not for stroke volume increases, that hypothesis may be rejected. Changes in the initial state of involved mechanisms, related to heart failure, rather than the amount of spared myocardium may account for the relationship between inotropic effects and infarct size, which is supported by experimental evidence.

The major mechanism of action by which milrinone causes enhanced contractility is accumulation of CAMP and increase of intracellular $\mathrm{Ca}^{2+}$. For the accumulation of CAMP three mechanisms have been proposed: (1) inhibition of phosphodiesterase which breaks down produced cAMP (Paton et al, 1988), (2) (at high doses) blockade of adenosine $A_{1}$ receptors, the activation of which inhibits CAMP production (Earl et al, 1986; Paton at al, 1988) and (3) direct blockade of the inhibiting guanine nucleotide regulatory protein Gi (Parsons et al, 1987; Paton et al, 1988). In severe heart 
failure, the production of cAMP is impaired (Feldman et al; 1987). Besides downregulation of beta-receptors (Bristow et al, 1986), an increased release of adenosine (Earl et al, 1986) and increased Gi proteins (Böhm et al, 1989) in heart failure could be responsible for this impaired cAMP production. Thus, accumulation of CAMP due to milrinone is likely to be impaired related to severity of heart failure.

Furthermore, the inotropic effects of milrinone are influenced by extracellular $\mathrm{Ca}^{2+}, \mathrm{Na}^{+}$and $\mathrm{K}^{+}$concentrations (Farah et al, 1988) because these ion concentrations can affect intracellular $\mathrm{Ca}^{2+}$. Effects of milrinone are suggested to be increased at low intracellular $\mathrm{Ca}^{2+}$ concentrations whereas at higher concentrations they may be decreased (Farah et al, 1988). In mild heart failure intracellular $\mathrm{Ca}^{2+}$ can be reduced (Feldman et al, 1987) whereas in severe heart failure it may be increased (Meerson, 1983). This suggests an increased response to milrinone in mild heart failure and a decreased response in more severe heart failure. The overstimulation at small infarct sizes compared to rats without infarct could then be explained on the basis of a low intracellular $\mathrm{Ca}^{2+}$ because ventricles of healthy rats act at a near maximal $\mathrm{Ca}^{2+}$ transport over the membranes (Alousi et al, 1988; Sys et al, 1986). Reduction of inotropic responses to milrinone at greater infarct size could be attributed to an increased intracellular $\mathrm{Ca}^{2+}$.

Finally, the stimulatory effect of cAMP on calcium activated contraction is reduced when cardiac myosin isoenzyme pattern is shifted to V3 type fibers (Horowits et al, 1987). Such a shift is reported after myocardial infarction in rats (Geenen et al, 1989) and may be related to the severity of heart failure.

The above-mentioned effects of milrinone could contribute to the dose-dependent improvement after 2 weeks therapy; all hemodynamic changes due to infarction were restored. The different effects may even facilitate each other. Increasing cAMP and a better handling of intracellular $\mathrm{Ca}^{2+}$ in myocytes would result in improvement of contraction and relaxation by an increased release and re-uptake of $\mathrm{Ca}^{2+}$ in the sarcoplasmic reticulum (Sys et al, 1986; Evans, 1989). As a result, $\mathrm{Ca}^{2+}$ would be released from mitochondria increasing oxidation phosphorylation (Meerson et $\mathrm{al}$, 1983). In addition, a facilitation of the response to adrenergic stimulation (Colucci, 1989; Verrijk et al, 1989) might improve adrenergic regulation of contractility. A preferential coronary vasodilation (Harris et al, 1989) would increase oxygen supply.

However, not only the cardiac effects are important, as is illustrated by the differences between intracoronary milrinone administration compared to intravenous administration (Colucci, 1989). Reduced afterload and preload with milrinone limited the increase in $\mathrm{O}_{2}$ consumption due to increased contractility (Baim, 1989). The increase in $\mathrm{CO}$ was predominantly distributed to the coronary circulation and to skeletal muscle in man (Harris et al, 1989; LeJemtel et al, 1989) and in infarcted rats (Drexler et al, 1987b) and, hence, increased exercise tolerance (LeJemtel et al, 1986). However, despite an increase in cardiac output, neither in man (Cody et al, 1986) nor in rats (Drexler et al, 1987b) impaired renal blood flow was increased. In man, also renal function was not changed by milrinone (Cody et al, 1989).

In the present study, the effects found after 2 weeks milrinone indicate a clear increase in contractility, which is in accordance with data of Emmert et al (1987) in heart failure rats. This results in a significant improvement of cardiac function. In man, chronic milrinone can also cause hemodynamic improvement measured as increased $\mathrm{Cl}$ and decreased pulmonary pressure and left ventricular end-diastolic pressure. Effects were sustained as long as milrinone was administered but returned 
to pretherapeutic walues after withdrawal (LeJemtel et al, 1984; Baim et al, 1984; Maskin et al, 1984; Monrad et al, 1986). No tachyphylaxis was found in man nor in rats (Enmert et al, 1987). The lack of sustained improvement after withdrawal in man suggests no effects on long-term regulatory mechanisms. Regression of cardiac hypertrophy as might have been indicated by decreased heart weight to body weight ratio was not observed in the present study, although decreased heart weight and QS duration suggested a decreased theart size. In addition, the lack of effects on left ventricular dilatation despite possible preload reduction may support the hypothesis that no structural improvement has occurred.

Despite the hemodynamic improvement in rats and in man, only in rats an increased survival has been reported (Sweet et al, 1988). In man mortality may be even higher (DiBianco et al, 1989) probably due to increased arrhythmias caused by milrinone (Holmes et al, 1985; DiBianco et al, 1989), although no increase in arrhythmias has been reported as well (Baim et al, 1986; Ludmer et al, 1987). An explanation could be interference with effects of digitalis and diuretics since both affect ion concentrations for which milrinone effects are sensitive. In rats milrinone given as monotherapy but also combined with enalapril increases survival (Sweet et al, 1988). Furthermore, increased responses to catecholamines after milrinone, in addition to the lack of reduction of circulating catecholamine levels (Cody et al, 1986) can also contribute to the arrhythmogenic effects of milrinone.

In conclusion, acute milrinone in normal healthy rats has dose-dependent vasodilating and positive inotropic effects. Comparable effects were found in chronically infarcted rats. Two weeks milrinone significantly restored cardiac function without effects on MAP or HR. The long-term effect was achieved by a dose which presumably has no acute inotropic effects. 
chapter 6

DOBUTAMINE IN HEART FAILURE

\subsection{Introduction}

Stimulation of the sympathetic nervous system is a physiological way to increase cardiac output through stimulation of alpha- and beta-adrenoceptors. $( \pm)$ Dobutamine is a synthetic catecholamine racemate. The $(+)$ stereoisomer stimulates beta 1 and beta-2 receptors, whereas the (-)stereoisomer predominantly stimulates alpha- 1 receptors (Hayes et al, 1985). ( \pm )Dobutamine racemate henceforth will be referred to as dobutamine. It has predominantly positive inotropic effects with rather small tachycardia. Also, systemic vascular effects are small as compared to those of other catecholamines like noradrenaline, isoprenaline and dopamine (Ruffolo, 1987).

In heart failure, cardiac function is depressed. Down-regulation of beta-receptors (Bristow et al, 1986) and impaired up-regulation during physical exercise (Leinonen et a], 1987) together with increased circulating catecholamine levels implies the loss of an important regulatory mechanism. Despite the decreased adrenergic responsiveness in heart failure (Brown et al, 1986; Feldman et al, 1987) acute dobutamine infusion has been found to improve hemodynamics (Liang et al, 1984). However, long-term therapy is limited by the development of tolerance, already after 3 days of infusion (Unverferth et al, 1980). To prevent this, clobutamine can be administered intermittently (Mauro et al, 1987; Thomas et al, 1987). Beneficial hemodynamic effects and increased exercise tolerance have been found repeatedly, even though studies differ in details of their design, such as the lack of suited control groups, small numbers of patients (1-3), selection of patients and different therapeutic regimens. Moreover, after discontinuation of infusion, improvement was sustained (Unverferth et al, 1980) suggesting changes in long-term regulatory systems.

In the present study, hemodynamic effects of intermittent dobutamine therapy at 2 doses were investigated in rats with heart failure. From 3 to 5 weeks after infarction, when healing processes are complete (Fishbein et al, 1981) and heart failure development is rather stable (chapter 4), rats received i.p. injections of dobutamine twice daily with at least $90 \mathrm{~min}$ in between (Davidson et al, 1986). Changes in responsiveness, like down-regulation, may occur due to infarction or due to the 2 weeks of dobutamine therapy. Therefore, hemodynamic responses to acute dobulamine infusion in the 2 weeks dobutamire treated and non-treated infarcted rats were compared to those in normal healthy rats. 


\subsection{Experimental protocols}

Experiments were performed in male Wistar rats, weighing $250-350 \mathrm{~g}$ at the start of the protocol. Hemodynamic effects of dobutamine were studied in 3 experiments. In normal healthy rats, acute effects were studied to verify positive inotropic effects. Effects of 2 weeks intermittent dobutamine therapy were investigated in rats with heart failure and results were compared to sham control and non-treated infarcted control rats. In addition, hemodynamic responses to acute dobutamine infusion were studied in non-treated infarcted rats and in infarcted rats after 2 weeks dobutamine therapy, in order to investigate functional changes in responsiveness.

\subsubsection{Acute effects in normal healthy rats}

\section{Surgery protocol}

Rats were anesthetized with pentobarbital and an electromagnetic flow probe was implanted (cf. section 2.4.1). Rats were allowed to recover 5-6 days from this surgery. Following the recovery period animals were reanesthetized with ether and an arterial catheter for MAP measurement and 2 venous catheters for infusions were implanted (cf. section 2.4.2, 2.4.4). Experiments started at least one day after this surgery.

\section{Measuring protocol}

Hemodynamic measurements were performed every other day in order to prevent effects of a previous volume load or drug infusion. In random order rats were subjected to control volume load or volume load during infusion of 20,60 or $200 \mu \mathrm{g} / \mathrm{kg}$.min dobutamine.

After connection to the measuring equipment rats were allowed to habituate for 45-60 min. Then, baseline values were obtained and either a control volume load was given (cf. 2.5.1) or infusion was started (total infused volume $<1 \mathrm{ml}$ ). During the infusion, when hemodynamics had stabilized or at least 30 min later, the heart was also maximally stimulated by a volume load. Hemodynamics were continuously monitored during the experiment (Grass polygraph).

\subsubsection{Two weeks therapy in rats with heart failure followed by acute administration}

\section{Surgery protocol}

Rats were subjected to coronary artery ligation and infarcts were allowed to heal. Four weeks later, electromagnetic flow probes were implanted. Following recovery for 5-6 days, catheters for MAP and CVP measurements and for infusion, and ECG electrodes were implanted. Hemodynamic measurements were performed at least one day after this surgery. 


\section{Treatment and measuring protocol}

Three weeks after coronary artery ligation, therapy was started. Rats received 0.2 or $1 \mathrm{mg} / \mathrm{kg}$ dobutamine i.p. twice daily (injected volume $<0.3 \mathrm{ml}$ ) with at least 90 $\mathrm{min}$ in between. Preliminary results indicate that hemodynamic responses to i.p. dobutamine (tachycardia) had completely disappeared $30-45 \mathrm{~min}$ after injection.

At least 90 min after the last dobutamine injection rats were connected to the measuring equipment and signals were fed into preprocessor and computer for on-line derivation of hemodynamic parameters. After equilibration for 45-60 min baseline values were obtained. Then, the heart was stimulated by volume loading to determine maximal cardiac output. Hemodynamics were monitored continuously till CVP had returned to baseline values or maximally $60 \mathrm{~min}$ after volume loading. Data were stored on a hard disk for later analysis. Two days later while therapy was continued, this volume loading protocol was repeated during $60 \mu \mathrm{g} / \mathrm{kg}$.min dobutamine infusion (cf. 6.2.1). After finishing the experiments, rats were killed for determination of infarct size (cf. 2.3).

\subsection{Results}

\subsubsection{Acute effects in normal healthy rats}

Hemodynamic effects of acute dobutamine infusion are summarized in table 6.1. Dobutamine dose-dependently reduced MAP and increased $\mathrm{CO}$ at baseline. Increase in CO was due to a pronounced tachycardia with only a slight increase of SV. Effects on baseline MAP, HR and CO were statistically significant at the highest dose. Stimulated $\mathrm{CO}$ was significantly increased even at $60 \mu \mathrm{g} / \mathrm{kg}$.min. The increase of stimulated $\mathrm{CO}$ at $200 \mu \mathrm{g} / \mathrm{kg}$.min was due to a significant tachycardia as well as a significant increase in SV.

Results indicate that dobutamine has dose-dependent positive inotropic, positive chronotropic and vasodilating effects. Since $60 \mu \mathrm{g} / \mathrm{kg} . \mathrm{min}$ resulted in a significant increase of stimulated $\mathrm{CO}$, this dose was used to investigate functional responsiveness in the following experiments.

\section{3 .2 Efrects of 2 weeks therapy in infarcted rats}

\section{General}

In the non-treated infarcted group as well as during the low dose therapy one rat died due to surgery, whereas at the high dlose all rats survived. After exclusion of data from rats with small infarcts $(<21 \%)$, groups are characterized in table 6.2 . Infarcts sizes were comparable for the groups. Body weights of infarcted rats were significantly lower compared to sham rats but no effects of therapy were found. In fact, none of the parameters was altered by therapy. Heart weight per body weight tended to be lower after treatment, indicating no additional hypertrophy. 
Table 6.1 Effects of acute dobutamine in normal healthy rats $(n=5-6)$. Baseline (c) and volume load, $V L$ (c) represent values for control volume load, whereas baseline $(0)$, baseline (d) and $V L$ (d) represent baseline values before infusion, during infusion, and stimulated values during infusion, respectively.

\begin{tabular}{|c|c|c|c|c|c|}
\hline Dose dobutamine & $\begin{array}{l}\text { Contr } \\
\text { Baseline } \\
\text { (c) }\end{array}$ & $\begin{array}{l}\mathrm{L} \\
V L \\
\text { (c) }\end{array}$ & $\begin{array}{l}\text { Drug } \\
\text { Baseline } \\
\text { (o) }\end{array}$ & $\begin{array}{l}\text { infusion } \\
\text { Baseline } \\
\text { (d) }\end{array}$ & $\begin{array}{l}\text { VL } \\
\text { (d) }\end{array}$ \\
\hline \multicolumn{6}{|l|}{$20 \mu \mathrm{g} / \mathrm{kg} \cdot \mathrm{min}$} \\
\hline $\begin{array}{ll}\text { MAP } & (\operatorname{mHg})_{1} \\
\mathrm{HR} & (\min ) \\
\mathrm{SV} & (\mu \mathrm{I}) \\
\mathrm{CO} & (\mathrm{mI} / \mathrm{min})\end{array}$ & $\begin{array}{l}105 \pm 1 \\
362 \pm 10 \\
252 \pm 10 \\
91 \pm 5\end{array}$ & $\begin{array}{l}113 \pm 3 \\
383 \pm 15 \\
374 \pm 13 \\
144 \pm 11\end{array}$ & $\begin{array}{r}107 \pm 2 \\
387 \pm 8 \\
244 \pm 8 \\
95 \pm 5\end{array}$ & $\begin{array}{l}104 \pm 2 \\
424 \pm 15 \\
266 \pm 8 \\
113 \pm 8\end{array}$ & $\begin{array}{l}105 \pm 5 \\
433 \pm 18 \\
380 \pm 14 \\
170 \pm 11\end{array}$ \\
\hline $\begin{array}{l}60 \mu g / \mathrm{kg} \cdot \mathrm{min} \\
\mathrm{MAP}(\operatorname{ming}) 1, \\
\mathrm{HR}(\mathrm{min}) \\
\mathrm{SV}(\mu \mathrm{l}) \\
\mathrm{CO}(\mathrm{mI} / \mathrm{min})\end{array}$ & $\begin{array}{l}121 \pm 2 \\
393 \pm 15 \\
267 \pm 28 \\
104 \pm 11\end{array}$ & $\begin{array}{l}120 \pm 5 \\
389 \pm 16 \\
369 \pm 26 \\
143 \pm 16\end{array}$ & $\begin{array}{l}115 \pm 8 \\
421 \pm 19 \\
253 \pm 23 \\
103 \pm 10\end{array}$ & $\begin{array}{l}105 \pm 3 \\
526 \pm 5 \\
265 \pm 26 \\
136 \pm 14\end{array}$ & $\begin{array}{l}96 \pm 5^{*} \\
488 \pm 3^{*} \\
408 \pm 30 \\
196 \pm 17^{+}\end{array}$ \\
\hline $\begin{array}{l}200 \mu g / \mathrm{kg} \cdot \min \\
\mathrm{MAP}(\mathrm{mmHg}) 1 \\
\mathrm{HR}(\mathrm{min}) \\
\mathrm{SV}(\mu \mathrm{l}) \\
\mathrm{CO}(\mathrm{ml} / \mathrm{min})\end{array}$ & $\begin{array}{l}110 \pm 2 \\
360 \pm 4 \\
281 \pm 11 \\
102 \pm 3\end{array}$ & $\begin{array}{l}113 \pm 4 \\
395 \pm 7 \\
383 \pm 16 \\
151 \pm 7\end{array}$ & $\begin{array}{l}111 \pm 2 \\
373 \pm 8 \\
280 \pm 12 \\
104 \pm 4\end{array}$ & $\begin{array}{l}85 \pm 4^{*} \\
486 \pm 10^{*} \\
305 \pm 13 \\
148 \pm 4^{*}\end{array}$ & $\begin{array}{l}82 \pm 4^{+} \\
456 \pm 9^{+} \\
45.4 \pm 11^{+} \\
207 \pm 5^{+}\end{array}$ \\
\hline
\end{tabular}

\footnotetext{
* Significantly different from baseline before infusion (baseline (o))

${ }^{+}$Significantly different from stimulated values $V L$ (c) of control volume load
}

\section{Hemodynamic effects}

Chronic effects of infarction can be characterized by a depressed PF, SV, SW and $\mathrm{CO}_{\text {, wheras }} \mathrm{PEP}$ and PEP/LVET are elevated at baseline. No effects were found on baseline MAP and CVP nor on HR.

Two weeks intermittent dobutamine administration restored all changes significantly, except PEP and PEP/LVET (table 6.3). This latter parameter suggests a still decreased ejection fraction. Baseline MAP and HR were not altered by dobutamine (fig. 6.1). After stimulation by volume loading, the depressed cardiac function curve due to infarction was dose-dependently restored by 2 weeks dobutamine (fig. 6.2). The increased peak cardiac output was achieved by an increased SV without changes in HR (fig. 6.3). Indeed, also depressed $\mathrm{dF} / \mathrm{dt}$ and PF after stimulation were restored (table 6.4). Thus, 2 weeks intermittent dobutamine dose-dependently improved cardiac function by increasing contractility. An increased SV accounted for the improved cardiac performance since HR was not affected. 
Table 6.2 Characterization of the experimental groups

$\begin{array}{cc}\text { Sham Infarct } & \text { Dobutamine } \\ 2 \times 0.2 \mathrm{mg} / \mathrm{kg} * \mathrm{day} & \begin{array}{c}\text { Dobutanine } \\ 2 \times 1 \mathrm{mg} / \mathrm{kg} \cdot \mathrm{day}\end{array}\end{array}$

\begin{tabular}{|c|c|c|c|c|}
\hline$n$ & 7 & 8 & 7 & 7 \\
\hline inf. size $(\%)$ & 0 & $37 \pm 2^{+}$ & $37 \pm 2^{+}$ & $41 \pm 3^{7}$ \\
\hline $\mathrm{BW}(\mathrm{g})$ & $395+16$ & $326 \pm 13^{+}$ & $313 \pm 12^{*}$ & $335 \pm 8^{*}$ \\
\hline $\mathrm{HW}$ (mg) & $1352 \pm 163$ & $1284 \pm 137$ & $1066 \pm 69$ & 1169495 \\
\hline $\mathrm{HW} / \mathrm{BW} \quad(\mathrm{mg} / \mathrm{g})$ & $3.5 \pm 0.4$ & $3.8 \pm 0.4$ & $3.4 \pm 0.2$ & $3.5 \pm 0.3$ \\
\hline Resp. $\left(\min { }^{-1}\right)$ & $112 \pm 10$ & $147 \pm 11^{*}$ & $151 \pm 15^{+}$ & $140 \pm 10$ \\
\hline LVEC $(m m)$ & $7.8 \pm 0.6$ & $15.6 \pm 1.4^{+}$ & $16.7 \pm 1.5^{4}$ & $16.8 \pm 1.4^{+}$ \\
\hline
\end{tabular}

Abbreviations: inf. size: infarct size; $B$ : body welght; HW: heart weight; resp.: respiration rate; LVEC: mean left ventricular endocardiall circumference

${ }^{+}$Significantly different from sham operated rats
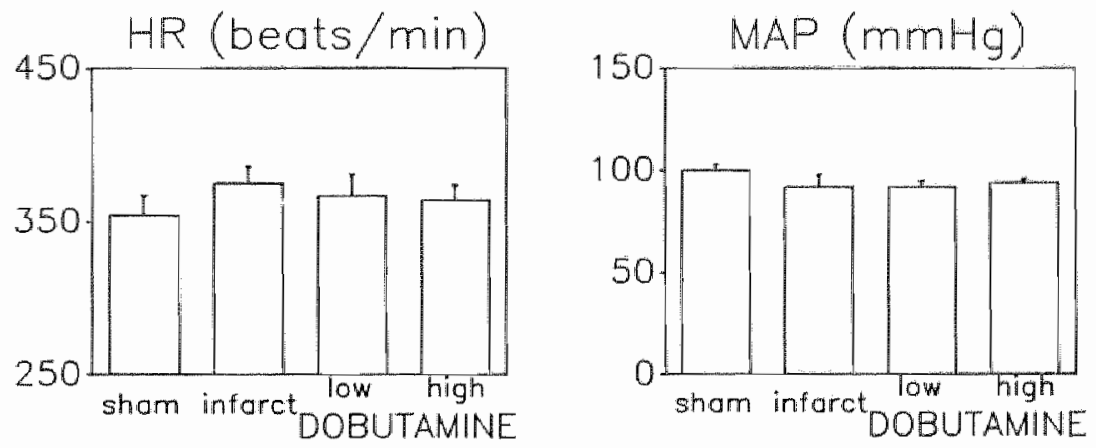

Fig. 6.1 Effects of 2 weeks intermittent dobutamine on baseline MAP and HR 
Table 6.3 Baseline hemodynamics after two weeks dobutamine therapy compared to non-treated infarcted rats and sham rats

Sham

7

n

$\mathrm{d} E / \mathrm{dt}\left(\mathrm{mi} / \mathrm{s}^{2}\right)$

$421 \pm 23$

$366 \pm 19$

$\mathrm{PF}$ (mi/min)

$0.9 \pm 0.8$

TPR (mmHg.min/ml)

SV $(\mu 1)$

OS (nis)

PEP (ms)

PEP/LVET

SW $(m \mathrm{mHg} \cdot \mathrm{ml})$
Infaret

Dobutamine $2 \times 0.2 \mathrm{mg} / \mathrm{kg}$. day
Dobutamine

$2 \times 1$ ing/ $/ \mathrm{kg}$. day

Abbreviations: $d F / d t$ : aortic flow acceleration; PF: peak flow; CVP: central venous pressure; TPR: total peripheral resistance; SV: stroke volume; QS: duration of QRS complex; PEP: pre-ejection period; PEP/LVET: ratio PEP and left ventricular ejection time; SW: stroke work

* Significantly different from sham control

*ignificantly different from infarct control

Table 6.4 Sitimulated values after two weeks dobutamine therapy

\begin{tabular}{|c|c|c|c|c|}
\hline & Sham & Infarct & $\begin{array}{l}\text { Dobutamine } \\
2 \times 0.2 \mathrm{mg} / \mathrm{kg} \text {. day }\end{array}$ & $\begin{array}{l}\text { Dobutamine } \\
2 \times 1 \mathrm{mg} / \mathrm{kg} \text {. day }\end{array}$ \\
\hline in & 7 & $B$ & 7 & 7 \\
\hline $\mathrm{dF} / \mathrm{dt}$ & $543 \pm 17$ & $406 \pm 30^{+}$ & $494 \pm 16^{*}$ & $557 \pm 21^{*}$ \\
\hline $\mathrm{PF}$ & $451 \pm 16$ & $348 \pm 18^{*}$ & $400 \pm 13$ & $469 \pm 16^{*}$ \\
\hline $\mathrm{PEP}$ & $33_{ \pm 1}$ & $38 \pm 2^{*}$ & $34 \pm 3$ & $33 \pm 2$ \\
\hline PEP/LUET & $0.40 \pm 0.01$ & $0.48 \pm 0.02^{+}$ & $0.41 \pm 0.05$ & $0.42 \pm 0.02$ \\
\hline SW & $34 \pm 1$ & $20 \pm 2^{*}$ & $26 \pm 2$ & $31 \pm 1^{\star}$ \\
\hline
\end{tabular}

For abbreviations and explanation of units, see table 6.3 


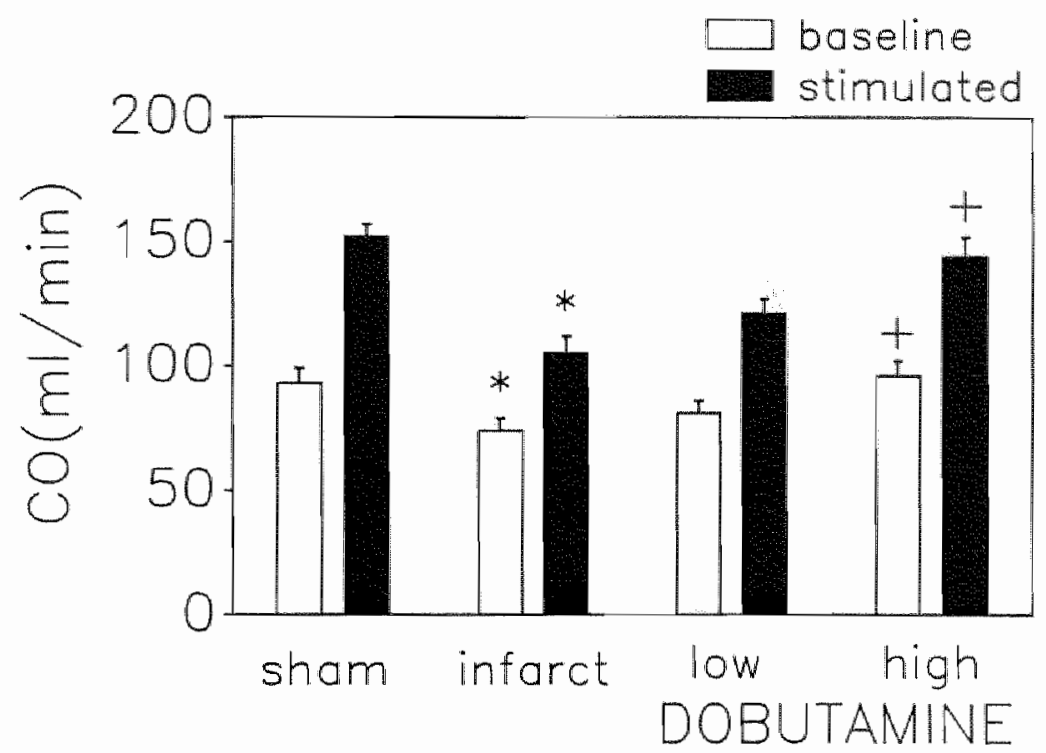

* significantly different from sham control

* significantly different from infarct contral

Fig. 6.2 Effects of 2 weeks intermittent dobutamine on baseline and stimulated $\mathrm{CO}$
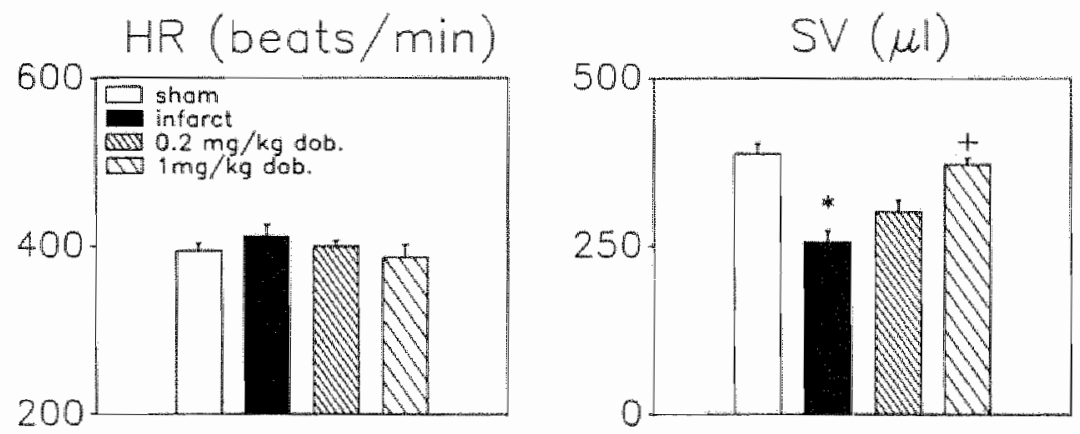

Fig. 6.3 Effects of 2 weeks intermittent dobutamine on stimulated HR and SW. For symbols see explanation figure 6.2 

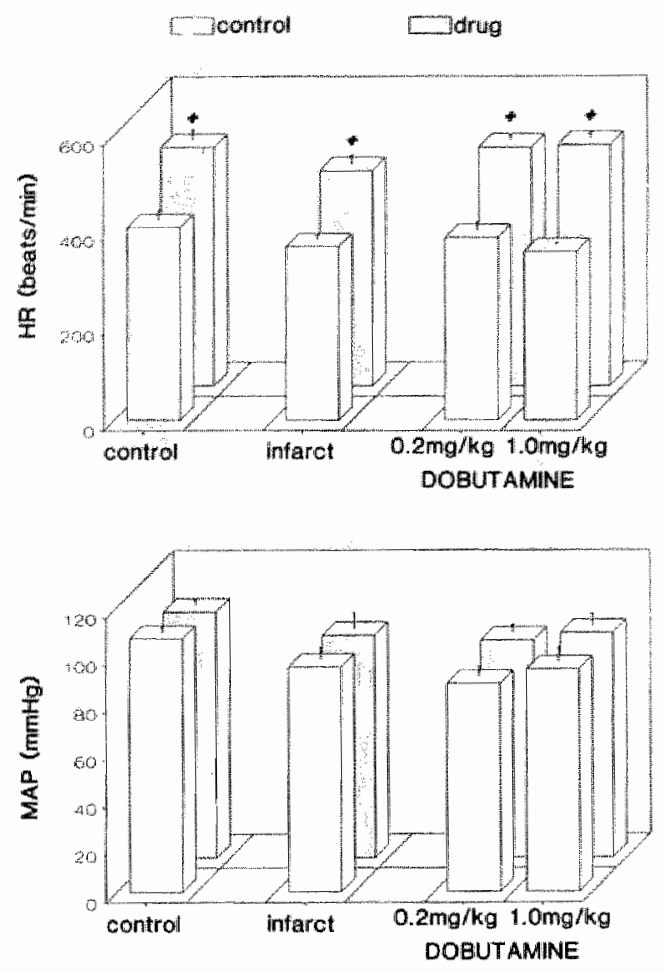

Fig. 0.4 Effects of acule doburamine infusion $60 \mu \mathrm{g} / \mathrm{kg}$ min following 2 weeks intermitfont dobutamine therapy on baseline MAP and HR

\section{3 .3 Effects of acute dobutamine after 2 weeks of therapy}

Effects of acute dobutamine were investigated to determine the influence of chronic infarction and long-term therapy on the functional response to dobutamine. Effects on baseline MAP and HR for the different groups are presented in fig. 6.4. Acute dobutamine had no effect on MAP in any of the experimental groups. Tachycardia was comparable in all groups, suggesting that the chronotropic response to dobutamine was not affected by chronic infarction nor by 2 weeks intermittent dobutamine therapy.

In contrast, effects on cardiac function were affected as can be seen in figure 6.5. This figure presents, for the 4 experimental groups the control volume load and volume load during acute dobutamine infusion. In infarcted rats, the significant increase of baseline $\mathrm{CO}$ after acute dobutamine was comparable to that in control 


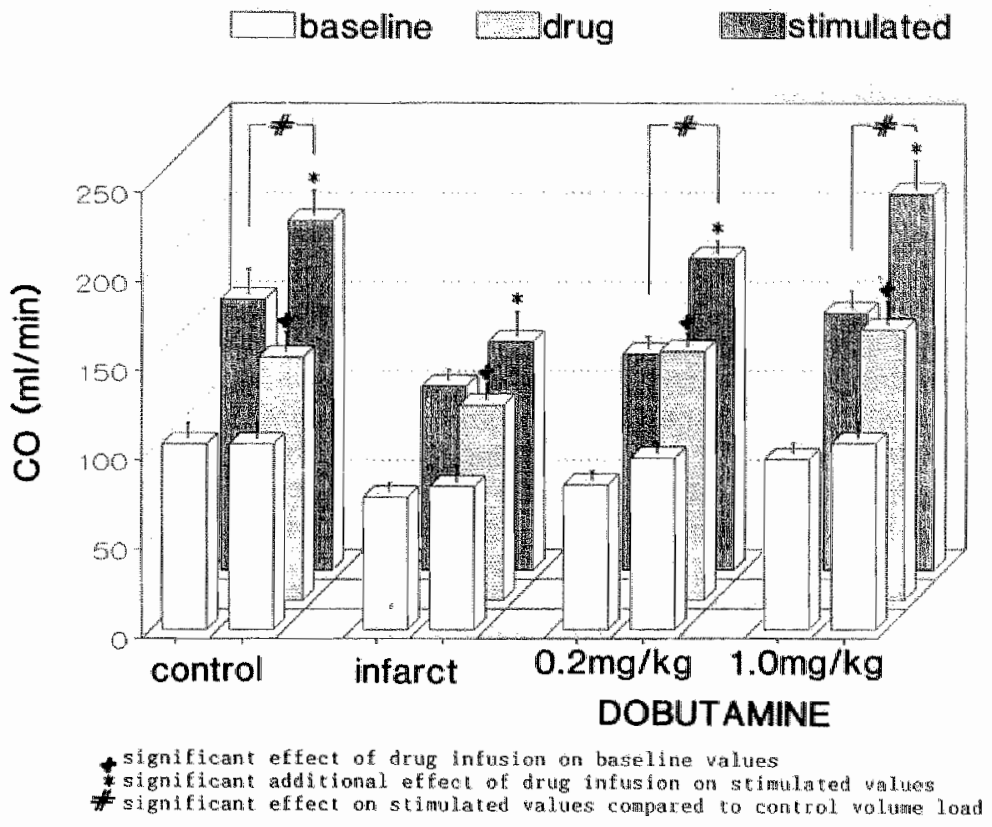

Fig. 6.5 Effects of acute dobutamine infusion after 2 weeks intermittent therapy on baseline and stimulated cardiac output

rats. This increase was slightly but insignificantly enhanced in long-term dobutamine treated rats.

In non-treated infarcted rats, volume load during acute dobutamine infusion increased $\mathrm{CO}$ slightly but significantly in addition to the increased baseline level. Maximally stimulated $\mathrm{CO}$ during acute dobutamine infusion was, however, not significantly higher than without dobutamine infusion. This additional increase of $\mathrm{CO}$ after volume load, which was significantly depressed due to infarction, was significantly restored by long-term dobutamine therapy.

After long-term treatment, the significant increase of stimulated $\mathrm{CO}$ after acute administration compared to control volume load was due to increased HR and SV. It was not related to infarct size.

Thus, long-term dobutamine therapy preserved the baseline responses to acute adminstration of dobutamine and reversed the depressant effects of infarction on stimulated $\mathrm{CO}$ in a dose-dependent way. 


\subsection{Disscussion}

( \pm )Dobutamine is a synthetic catecholamine racemate which stimulates beta-1, beta-2 and alpha-1 adrenoceptors in the cardiovascular system (Ruffolo, 1987). Inotropic effects have been reported to be predominant over chronotropic effects and effects on peripheral resistance when compared to other catecholamines, like isoprenaline and dopamine (Ruffolo, 1987). Activation of beta-1 (Bristow et al, 1986; Ruffolo, 1987), beta-2 (Bristow et al, 1986; Leenen et al, 1988) and alpha-1 receptors (Böhm et al, 1988) can contribute to the inotropic effect whereas vasodilatation due to beta-2 receptor stimulation and vasoconstriction due to alpha-1 stimulation may result in rather small effects on peripheral resistance.

In the present study, in normal healthy conscious rats, acute dobutamine caused a dose-dependent decrease in MAP and an increase in HR without significant effects on SV. The lack of effect on baseline SV may be due to reduced left ventricular filling pressure. Compared to the effects of milrinone (chapter 5) which even caused a decreased $\mathrm{SV}$, a decreased right ventricular afterload with milrinone but not with dobutamine (Eichhorn et al, 1987) supports this hypothesis. When cardiac filling pressure was increased by volume load, inotropic effects of dobutamine became overt resulting in a significant increase in both peak SV and peak CO. Thus, in normal healthy rats acute dobutamine caused tachycardia and decreased peripheral resistance at baseline while positive inotropic effects increased cardiac performance.

The purpose of the present study was to investigate hemodynamic effects of 2 weeks intermittent dobutamine therapy in rats with heart failure. Although dobutamine improves the clinical situation after acute administration, its application for long-term therapy is limited because of tolerance development. After 72 and $96 \mathrm{hr}$, responses are diminished (Unverferth et al, 1980). After one week infusion, initially increased CI and SVI return to pre-infusion values or even lower (Kupper et al, 1.985). Intermittent administration may prevent this development of tolerance.

In the present study, dobutamine was injected i.p. at two different doses twice daily with at least $90 \mathrm{~min}$ in between. Hemodynamic measurements were also performed at least $90 \mathrm{~min}$ after the last injection. Since hemodynamic effects are strongly related to plasma levels of dobutamine (Mauro et al, 1987), the observation that 30-45 min after injection tachycardia had completely disappeared (present studly), and $d \mathrm{P} / \mathrm{dt}$ increases were not detectable anymore after 60-80 min (Dawidson et al, 1986) indicates no effective levels of circulating dobutamine at the moment of measurement.

Two weeks dobutamine in the present study had no effect on baseline MAP and $H R$, also indicating no effective circulating levels of dobutamine. Baseline $S V, S W$ and $\mathrm{CO}$, which were significantly depressed by infarction, improved dose-dependently. In fact, all hemodynamic changes at baseline due to infarction were restored or tended to be restored except for elevated PEP/LVET. The latter indicates a still reduced ejection fraction. Results correspond well with the data of Leier et al (1982) who treated patients for 24 weeks with $4 \mathrm{hr}$ weekly infusions. They also showed no effect on MAP, HR and PEP/LVET despite an increase in contractility. Exercise duration increased without an additional increase in heart rate. Cardiac output was not measured. 
In the present study, when the heart was stimulated by volume loading cardiac performance was improved. This was due to increased SV, indicating increased contractility, without increase in HR. Whereas baseline values were elevated, after stimulation PEP/LVET decreased to sham values. In most of the clinical studies (Applefeld et al, 1983; Hodgson et al, 1984; Berger et al, 1985; Roffman et al, 1985; Dies, 1986) patients received weekly $48 \mathrm{hr}$ dobutamine infusion. Despite differences in type of patients, study design and therapy periods between the above mentioned studies, hemodynamic effects are comparable: increased SV and $\mathrm{CO}$, decreased systemic vascular resistance without effects on systemic blood pressure or HR. This is in general accordance with the results in the present study.

A second important observation in the present study is that during intermittent dobutamine treatment, improvement was sustained without evidence for circulating dobutamine. In humans sustained improvement was illustrated in a study by Unverferth et al (1980) in which patients were followed up after 3 days intusion of dobutamine; $84 \%$ had symptomatic improvement 2 days after infusion, $59 \%$ was still improved at 4 weeks and $43 \%$ showed improvement at 10 weeks. Comparable results were found by Liang et al (1984). The sustained improvement after dobutamine without obvious circulating levels of the drug suggests changes in long-term regulatory systems.

In the present model at the time of start of the therapy structural changes like hypertrophy are present (Anversa et al, 1986; Fishbein et al, 1988). Although alpha(Simpson et al, 1988) and beta- stimulation (Tse et al, 1979) could induce myocardial hypertrophy, in the present study normalized HW/BW suggests regression of hypertrophy rather than induction. After myocardial infarction in rats, not only the number of myofibrils per myocyte increases (Anversa et al, 1986) but also a shift in myosin isoenzymes pattern from V1 to V3 fibers was found (Karam et al, 1988; Geenen et al, 1989). V3 fibers have a 3 times lower myosin ATP-ase activity and a lower oxygen demand while total force is similar to that of V1 fibers (Winegrad et al, 1987). Contractility is correlated with the ATP-ase activity and therefore determined by the ratio $\mathrm{V} 1 / \mathrm{V} 3$ at a given number of myofibrils whereas total force is determined by the total number of myofibrils (Rupp et al, 1987; Winegrad et al, 1987). When V1/V3 ratio is decreased this does not necessarily imply a depressed cardiac function since the total number of myofibrils may have increased (Winegrad et al, 1987). Perhaps a distinction should be made between "quantitative" hypertrophy, the conventional type with an increased number of myofibrils per myocyte, and "qualitative" hypertrophy with increasing V1/V3 ratio and therefore contractility. Both determine cardiac performance. Dobutamine can increase V1/V3 ratio (Rupp et al, 1986; Buttrick et al, 1988). An increase of the decreased V1/V3 ratio after infarction (Geenen et al, 1989) would explain many of the results in the present study:

- In the first place, the sustained improvement of cardiac performance without circulating dobutamine. V1 fibers have a higher ATP-ase activity and a higher velocity of fiber shortening or increased contractility (Winegrad et al, 1987). The present study indicates a higher contractility after 2 weeks therapy, resulting in an improved cardiac performance.

Secondly, the improved cardiac performance without additional increase in heart weight could be explained by a different proportion of qualitative to quantitative hypertrophy. Increased qualitative hypertrophy by increasing V1/V3 ratio could reduce quantitative hypertrophy by reducing the number of myofibrils, at maintained or even increased cardiac function. An addi- 
tional beneficial effect of reducing myocyte size includes restoration of maximal oxygen diffusion distance, cell surface/cell contents ratio, mitochondrium/myofibril ratio and innervation described in chapter 4 . Furthermore, dobutamine has been reported to increase coronary flow in infarcted rats (Drexler et al, 1987b) indicating a better oxygen supply. Since not only cardiac muscle but also skeletal muscle exhibits abnormalities in heart failure (Dunnigan et all, 1987; Massie et al, 1988; Lipkin et al, 1988), and also skeletal muscle flow is increased by dobutamine (Drexler et al, 1987b) comparable mechanisms might contribute to increased exercise tolerance.

- Finally, HR response to acute dobutamine was not changed by infarction or 2 weeks dobutamine therapy, whereas the inotropic response, measured as increased maximal $\mathrm{CO}$, was depressed due to infarction but restored by 2 weeks dobutamine therapy.

Reduced responsiveness to catecholamines is a common phenomenon in heart failure and may be due to an impaired second messenger system (Feldman et al, 1987; Brown et al, 1986) or decreased beta-adrenoceptor density (Bristow et al, 1982). However, 3 weeks after infarction, Clozel et al (1987) reported in rats no changes in affinity, and even an increase in number of beta-adrenergic binding sites with decreased norepinephrine content of the heart. In that study, HR response to isoprenaline was similar in sham and infarcted hearts but inotropic response was impaired due to infarction, indicating that effects can be separated. If the inotropic effects of dobutamine would be due to beta- $\mathbb{1}$ and the tachycardia to beta-2 receptor stimulation, then selective down-regulation of beta-1 receptors (Bristow et al, 1986) could explain the impaired inotropic, but not chronotropic response after infarction. However, this reduced inotropic effect with preserved HR response, was found when beta-receptors were actually reported not to be down-regulated (Clozel et al, 1987), indicating contribution of different mechanisms. A decreased V1/V3 ratio due to infarction could explain the discrepancy between HR and inotropic responses since V3 fibers can not be activated by beta-stimulation (Winegrad et al, 1987). Inotropic responses but not HR responses depend on contractile elements, indicating only the first being reduced by a decreased V1/V3 ratio. The highest dobutamine dose in the present study, $2 \times 1 \mathrm{mg} / \mathrm{kg}$, when given for 14 weeks in normal rats causes neither down-regulation nor changes in affinity of beta-receptors and no reduction of adenylate cyclase or $\mathrm{CAMP}$ production (Davidson et al, 1986). An increased V1/V3 ratio after 2 weeks dobutamine therapy may then explain the restored inotropic effect without changes in HR response. Moreover, acute dobutamine may not only increase the ATP-ase activity of V1 fibers but also inhibit the ATP-ase activity of V3 fibers, suggesting not only an increase in total ATP-ase activity but also an increase in the functional expression of the V1 fibers at the expense of the V3 fibers (Winegrad et al, 1987). Thus, an increased proportion of $V 1$ type fibers after dobutamine in the present study would not only explain the improved cardiac function after 2 weeks therapy but also the restored inotropic response to acute dobutamine after the 2 week therapy.

Although intermittent dobutamine clearly has beneficial effects, the question whether it also would increase survival remains uncertain. Sustained elevated PEP/LVET in the present study indicates sustained reduction of ejection fraction which, in rats, predicts survival (Pfeffer et al, 1987). Activity of the sympathetic nervous system, indicated by plasma noradrenaline levels, provides another important predictor of prognosis (Packer et al, 1987). Dobutamine did neither increase noradrenalline 
release from nerve terminals (Mauro et al, 1986), nor noradrenaline content of the myocardium (Davidson et al, 1986), nor circulating plasma noradrenaline (Liang et al, 1984) in heart failure. However, a severe increase in mortality, which was not restricted to the infusion periods and caused by pump failure as well as arrhythmias was reported by Dies (1986). The natural response to heart failure, consisting of a decreased V1/V3 ratio, reduced cAMP production and beta-receptor down-regulation could then be seen as a sensible way to reduce mortality (Katz, 1989) at the expense of exercise tolerance.

In conclusion, acute dobutamine administration caused tachycardia and inotropic effects. Intermittent dobutamine administration from 3 to 5 weeks after infarction in rats with heart failure, not only restored cardiac performance without additional increase in HR, but also restored inotropic response to adrenergic stimulation in a dose-dependent way. Although the mechanism of action is not completely understood, shifts in myosin isoenzyme pattern may provide an explanation for the observed effects. The study suggests that dobutamine has a long-term inotropic effect, different from the acute inotropic effect. 



\section{CAPTOPRIL IN HEART FAILURE}

\subsection{Introduction}

In the therapy of heart failure, stimulation of the heart by inotropic agents is one strategy to improve cardiac performance, while unlloading the impaired myocardium is another. The latter strategy is of growing interest since an increased survival has been reported for some vasodilating therapies (Cohn et al, 1986, 1987; Consensus Trial, 1987).

Myocardial infarction evokes activation of compensatory mechanisms which act to maintain systemic perfusion. These mechanisms include enhancement of sympathetic tone and activation of the renin-angiotensin-aldosteron system resulting in vasoconstriction and volume expansion (Parmley, 1985; Francis, 1985; Remme, 1986; Cody, 1986). While these responses may be beneficial in acute heart failure to help maintaining perfusion pressure, they could be detrimental in chronic stages of the disease (Parmley, 1985; Cody, 1986). Both vasoconstriction and volume expansion may be due to increased angiotensin II levels. Indeed, reduction of angiotensin II levels by angiotensin I converting enzyme (ACE) inhibition is considered to improve the clinical state in congestive heart failure (Cody, 1986; Cleland et al, 1986; "Timmis; et all, 1987; Pfeffer et al, 1988).

Also in rats with heart failure, chronic $\mathrm{ACE}$ inhibition results in hemodynamic improvement (Pfeffer et al, 1985a) and increased survival (Pfeffer et al, 1985b; Sweet et al, 1987). In the former study no differences in hemodynamic effects were found when therapy was started in the early phase of healing processes, 2 days after infarction, or when these processes are considered to be completed, 21 days after infarction (Fishbein et al, 1981; Pfeffer et al, 1979a), suggesting minor effects of ACE inhibition during the healing period. The effects, however, were measured after a long period of treatment which could mask effects of therapy during the healing period.

The aim of the present study was to compare hemodynamic effects of short-term captopril therapy in different periods following mycoardial infarction. Rats were treated with captopril during the healing period from $1-21$ days following infarction and after the healing period from 21-35 days after infarction. In addition, acute effects of captopril were studied after the repair perjod. 


\subsection{Experimental protocols}

Male Wistar rats, weighing $250-350 \mathrm{~g}$ at the start of the protocol, were used throughout this study. Hemodynamic effects of $\mathrm{ACE}$ inhibition were studied in three experiments. Acute effects of captopril were determined in infarcted rats 5 weeks after ligation. Effects of two weeks therapy after the healing period were studied at 5 weeks after ligation, whereas effects of captopril therapy during the healing period were studied at 3 weeks after ligation.

\subsubsection{Acute effects of captopril in chronically infarcted rats}

\section{Surgery protocol}

Rats were subjected to coronary artery ligation as described in section 2.2.1. Infarcts were allowed to heal. One week before hemodynamic measurements rats were anesthetized with pentobarbital and electromagnetic flow probes were implanted. After 5-6 days recovery catheters were implanted for MAP and CVP measurements and for infusions. In addition, ECG electrodes were implanted (cf. section 2.4.1-2.4.5). Experiments were started at least one day following this surgery.

\section{Measuring protocol}

Hemodynamic measurements were performed at day 1 and 3 of the protocol. Rats were subjected to control volume load and to volume load after captopril in random order. Animals were connected to the measuring equipment and signals were fed into the "real-time data processing system" (cf. section 2.5). After 45-60 min, baseline values were obtained. Then either a control load was given or $10 \mathrm{mg} / \mathrm{kg}$ captopril was injected i.v. (maximally injected volume $<50 \mu \mathrm{l}$ ). After $30 \mathrm{~min}$ ACE inhibition was verified by measuring TPR responses to $150 \mathrm{ng} / \mathrm{kg}$ angiotensin I which is a submaximal dose (pilot study). When hemodynamics had returned to pre-Ang I values, or maximally 5 min after the Ang I injection, $\mathrm{CO}$ was stimulated by volume loading. Hemodynamics were monitored continuously till CVP had returned to baseline values or maximally $60 \mathrm{~min}$ after volume load. Data were stored on a hard disk for later analysis. After finishing the experiments, rats were killed for determination of infarct sizes (cf. 2.3).

\subsubsection{Effects of two weeks captopril after the healing period}

\section{Surgery protocol}

The surgical preparation for hemodynamic measurements was similar to that described in section 7.2.1. In addition, 3 weeks after ligation under light anesthesia, osmotic minipumps (Alzet 2001, Palo Alto, USA) filled with captopril solution were implanted subcutaneously between the shoulder blades. Captopril was administered at rates of 100 or $500 \mu \mathrm{g} / \mathrm{kg}$.hr. After one week, when electromagnetic flow probes were implanted, pumps were replaced. 


\section{Measuring and therapy protocol}

Hemodynamic measurements were performed after 2 weeks therapy. Control infarcted and control sham rats received no therapy.

Rats were connected to the measuring equipment and signals were fed into preprocessor and computer for on-line derivation of hemodynamic parameters. After 45.60 min equilibration baseline values were obtained and ACE inhibition was verified by measuring TPR responses to $150 \mathrm{ng} / \mathrm{kg}$ Ang I. When hemodynamics had returned to pre-Ang I values, $\mathrm{CO}$ was maximally stimulated by volume loading. Hemodynamics were monitored till CVP had returned to its baseline value or maximally 60 min after volume load. Data were stored on a hard disk for later analysis. Rats were killed to determine infarct sizes (cf. 2.3).

\subsubsection{Effects of captopril therapy during the healing period}

\section{Surgery protocol}

The surgical preparation for hemodynamic measurements was similar to that described in section 7.2.1, except that effects were measured at 3 weeks in stead of at 5 weeks after ligation. Osmotic minipumps (Alzet 2001, Palo Alto, USA) filled with captopril solution were implanted as described in 7.2.2 one day after ligation and replaced every week.

\section{Measuring and therapy protocol}

Hemodynamic measurements were performed 3 weeks after ligation in treated and non-treated infarcted rats. Treated rats received $500 \mu \mathrm{g} / \mathrm{kg} . \mathrm{hr}$ captopril subcutaneously from 1 to 21 days after ligation.

Rats were connected to the measuring equipment and measurements were performed as described in 7.2.2.

\subsection{Results}

\subsubsection{Acute effects of captopril in chronically infarcted rats}

Thirty minutes after a bolus injection of $10 \mathrm{mg} / \mathrm{kg}$ captopril, verification of ACE inhibition indicated a significant depression of TPR response to $150 \mathrm{ng} / \mathrm{kg}$ Ang I ( $4 \pm 2 \%$ compared to control $41 \pm 8 \% ; \mathrm{p}<0.001$ ).

The only significant hemodynamic effects of acute captopril were decreases of MAP $(90 \pm 3$ to $81 \pm 4 \mathrm{mmHg} ; \mathrm{p}<0.05)$ and TPR $(1.00 \pm 0.05$ to $0.8 \pm \mathrm{m} 0.04 \mathrm{mmHg}$.$\mathrm{min} / \mathrm{ml} ; \mathrm{p}<0.05)$. Despite this reduction in afterload, cardiac function was not significantly affected as shown in fig. 7.1.

\subsubsection{Effects of 2 weeks captopril after the healing period}

\section{General}

Acute mortality caused by coronary artery ligation is described in chapter 4 . During low-dose captopril therapy, 4 rats died whereas at the high dose 1 rat died. 


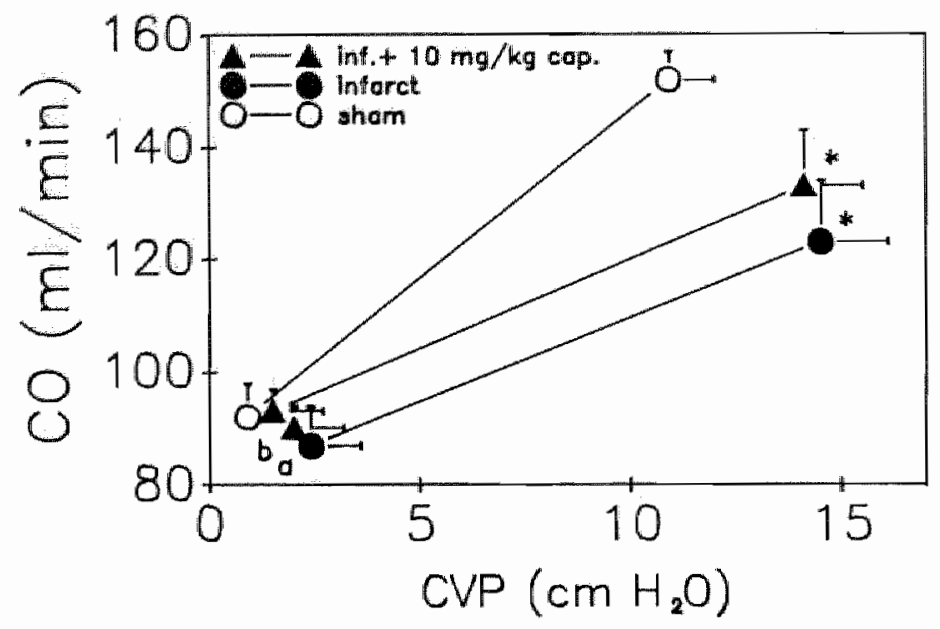

Fig. 7.1 Cardiac function after acute captopril treatment ( $-\star$ ) compared to cardiac function without treatment in the same rats $(\bullet-\bullet)(n=11)$ and to cardiac function in sham rats $(o-o)(n=7)$. Only baseline and maximally

stimulated values are presented. (a) and (b) present baseline values before and after a bolus injection of $10 \mathrm{mg} / \mathrm{kg}$ captopril, respectively.

Table 7.1 Characterization of experimental groups for therapy from 3.5 weeks after ligation

\begin{tabular}{|c|c|c|c|}
\hline sham & infarct & $\begin{array}{l}\text { captopril } \\
100 \mu / \mathrm{kg} \cdot \mathrm{hr}\end{array}$ & $\begin{array}{l}\text { captopril } \\
500 \mu \mathrm{g} / \mathrm{kg} \cdot \mathrm{hr}\end{array}$ \\
\hline
\end{tabular}

\begin{tabular}{|c|c|c|c|c|}
\hline$n$ & 7 & 8 & 7 & 7 \\
\hline inf. $t z e(\%)$ & 0 & $37 \pm 2^{+}$ & $36 \pm 3^{+}$ & $41 \pm 4^{+}$ \\
\hline BH $(g)$ & $395 \pm 16$ & $326 \pm 13^{t}$ & $300 \pm 8^{+}$ & $294 \pm 14^{*}$ \\
\hline$H W(m g)$ & $1352 \pm 163$ & $1284 \pm 137$ & $1037 \pm 48$ & $1125 \pm 49$ \\
\hline $\mathrm{HH} / \mathrm{BW}$ (mg/g) & $3.4 \pm 0.4$ & $3.8 \pm 0.4$ & $3.5 \pm 0.2$ & $3.9 \pm 0.2$ \\
\hline resp. $\left(\min ^{-1}\right)$ & $112+10$ & $147 \pm 11^{*}$ & $132 \pm 14$ & $136 \pm 13$ \\
\hline LVEC (min) & $7.8_{ \pm} 0.6$ & $15.6 \pm 1.4^{+}$ & $16.2 \pm 0.7^{+}$ & $15.8 \pm 1.0^{+}$ \\
\hline
\end{tabular}

Abbreviations: inf. size: infaret size; BW: body weight; HW: heart weight; resp.: respiration rate; LVEC: mean left ventricular endocardial circumference.

* Significantly different from non-treated infarcted rats + Significantly different from sham operated rats 
After exclusion of data from rats with infarct $<21 \%$, groups had comparable infarct sizes. Groups are characterized in table 7.1.

In infarcted groups, BW was significantly lower than in sham animals but no differences due to therapy were found. Also the ratio of heart weight to body weight was not significantly altered. Captopril did not significantiy change tachypnea or left ventricular dilatation caused by infarction.

ACE activity measured as TPR response to a submaximal dose of $150 \mathrm{ng} / \mathrm{kg}$ Ang I resulted for sham controls and infarct controls in a TPR increase of $41 \pm 17 \%$ and $41 \pm 8 \%$, respectively. In the treated groups the response was significantly reduced (llow dose: $24 \pm 6 \%$, high dose: $23 \pm 5 \%$ ), indicating long-term ACE inhibition.

\section{Hemodynamic effects}

Hemodynamic effects of 5 weeks infarction are described in detail in chapter 4. When small infarcts were excluded, depression of cardiac performance was significantly present even at baseline conditions (fig. 7.3). After stimulation by volume loading effects of infarction became more pronounced. DF/dt, PF, SV, SW and CO were depressed while PEP and PEP/LVET were increased. MAP and HR were not altered (fig. 7.2). 7.2).

Captopril dose-dependently reduced MAP without significant effects on HR (fig.
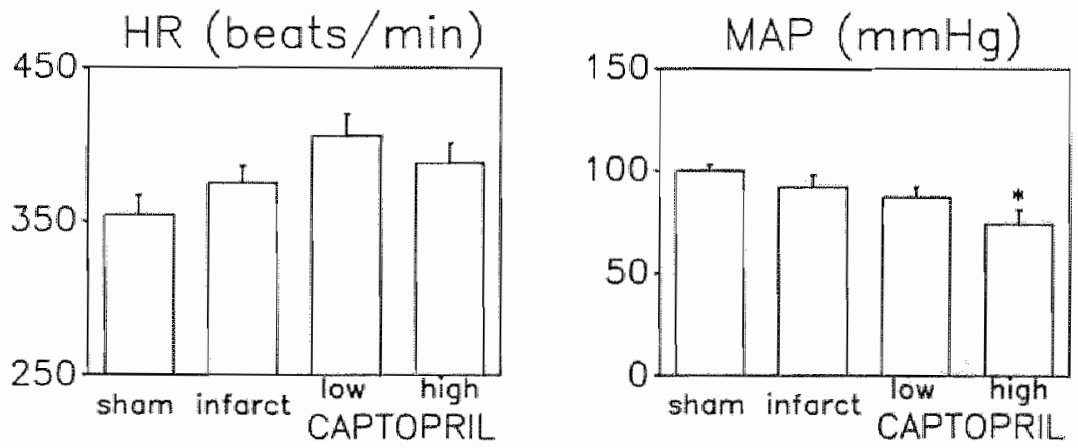

Fig. 7.2 Effects of captopril therapy from 3-5 weeks after infarction on baseline MAP and $H R$. 
Table 7.2 Baseline hemodynamics for the experimental groups

\begin{tabular}{|c|c|c|c|c|}
\hline : & sham & infaret & $\begin{array}{l}\text { captopril } \\
1.00 \mu g / \mathrm{kg} \cdot \mathrm{hr}\end{array}$ & $\begin{array}{l}\text { captopril } \\
500 \mu \mathrm{kg} / \mathrm{kg} \cdot \mathrm{hr}\end{array}$ \\
\hline$n$ & 7 & 8 & 7 & 7 \\
\hline$d P / d t\left(m 1 / s^{2}\right)$ & $421 \pm 23$ & $339 \pm 30$ & $374 \pm 32$ & $418 \pm 40$ \\
\hline $\mathrm{PP}(\mathrm{m} 1 / \mathrm{min})$ & $366 \pm 19$ & $285 \pm 24^{*}$ & $315 \pm 27$ & $353 \pm 23$ \\
\hline SV $(\mu 1)$ & $257+16$ & $199 \pm 17^{*}$ & $195+15$ & $253 \pm 19$ \\
\hline TPR (mmHg & $1.12 \pm 0.10$ & $1.25 \pm 0.15$ & $1.13 \pm 0.08$ & $0.78 \pm 0.07^{\star}$ \\
\hline $\operatorname{PEP}$ (ms) & $36 \pm 1$ & $40 \pm 1^{+}$ & $38 \pm 2$ & $37 \pm 2$ \\
\hline PEP/LVET & $0.49+0.01$ & $0.54 \pm 0.02^{+}$ & $0.56 \pm 0.03$ & $0.52 \pm 0.03$ \\
\hline $\mathrm{SW}(\mathrm{mmHg} \cdot \mathrm{mL})$ & $25+1$ & $18 \pm 1^{+}$ & $17 \pm 2$ & $19 \pm 2$ \\
\hline as (ms) & $16.7 \pm 0.9$ & $17.0+1.9$ & $15.5 \pm 1.5$ & $14 \cdot 2 \pm 1.2^{*}$ \\
\hline
\end{tabular}

Abbreviations: $d F / d t$ : aortic flow acceleration; PF: peak flow: SV: stroke volume; TPR: total peripheral resistance; PEP: pre-ejection period; LVET: left ventricular ejection time; SW: stroke vork; QS: width of QS interval.

* Significant effects of infarction

* Significant effects of therapy

Although depressed baseline SV and dF/dt were improved by captopril, effects were not significant (table 7.2). However, baseline $\mathrm{CO}$ was increased significantly (fig. 7.3) as compared to non-treated animals while TPR decreased dose-dependently. CVP was not altered by captopril. Indicators of mechancial properties of the heart PEP, PEP/LVET and SW were not significantly changed due to therapy (table 7.2). Moreover, SW remained significantly depressed compared to sham rats. QS decreased at the high dose.

Effects on cardiac performance after volume loading are shown in fig. 7.3. Captopril dose-dependently improved cardiac performance reaching statistical significance at the high dose. Contributions of SV and HR to the stimulated $\mathrm{CO}$ are summarized in fig. 7.4 which also presents effects on stimulated SW. Effects on stimulated $\mathrm{CO}$ were reflected in effects on SV although no statistical significances were achieved. SW was not affected by captopril therapy.

The improved cardiac performance after captopril could be due to the reduced afterload. To test this hypothesis, relationship between stimulated CO and MAP was analyzed using linear regression (fig. 7.5). This correlation was found to be positive, suggesting better cardiac performance at higher MAP or alternatively, higher MAP at better performance. 


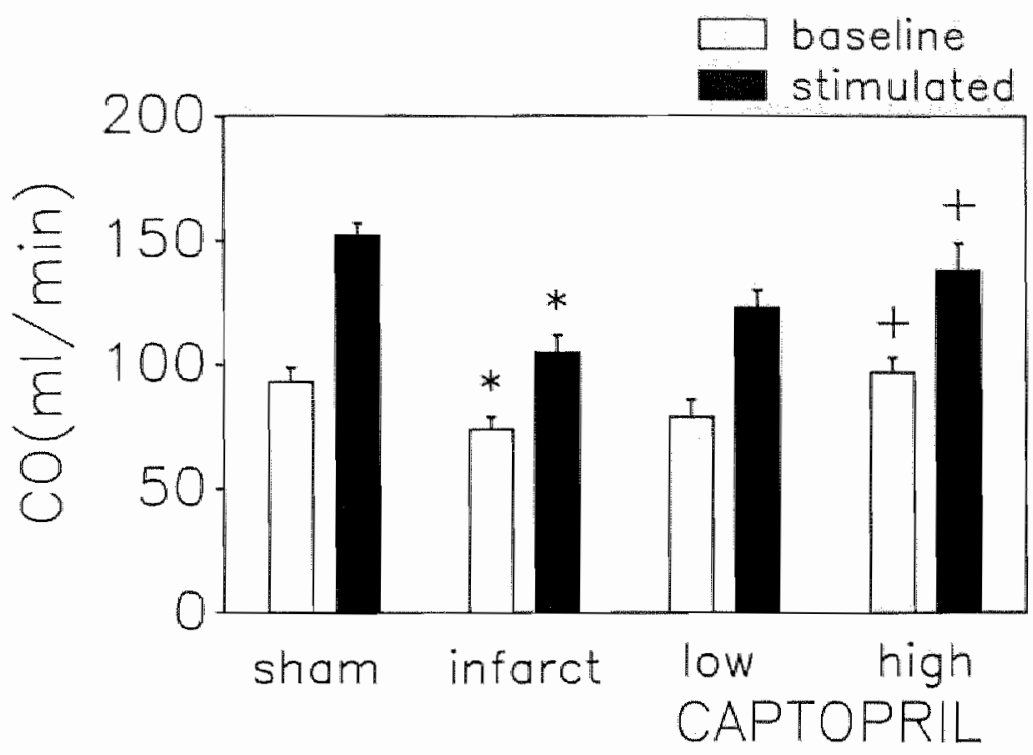

* significantly different from sham control

+ significantly different from infarct control

Fig. 7.3 Effects of captopril therapy from 3-5 weeks after infarction on baseline and stimulated $\mathrm{CO}$.
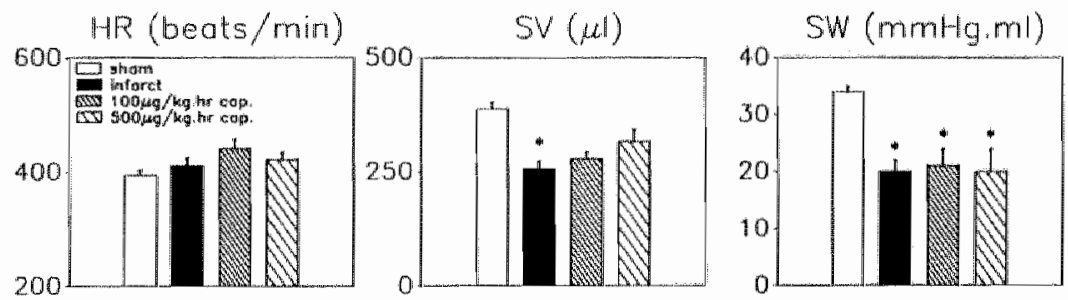

Fig. 7.4 Contribution of $H R, S V$ and $S W$ to the stimulated $C O$ after short-term captopril therapy from 3-5 weeks after infarction. 
Thus, short-term captopril dose-dependently improved cardiac performance. The improvernent could not be attributed to a decreased MAP.

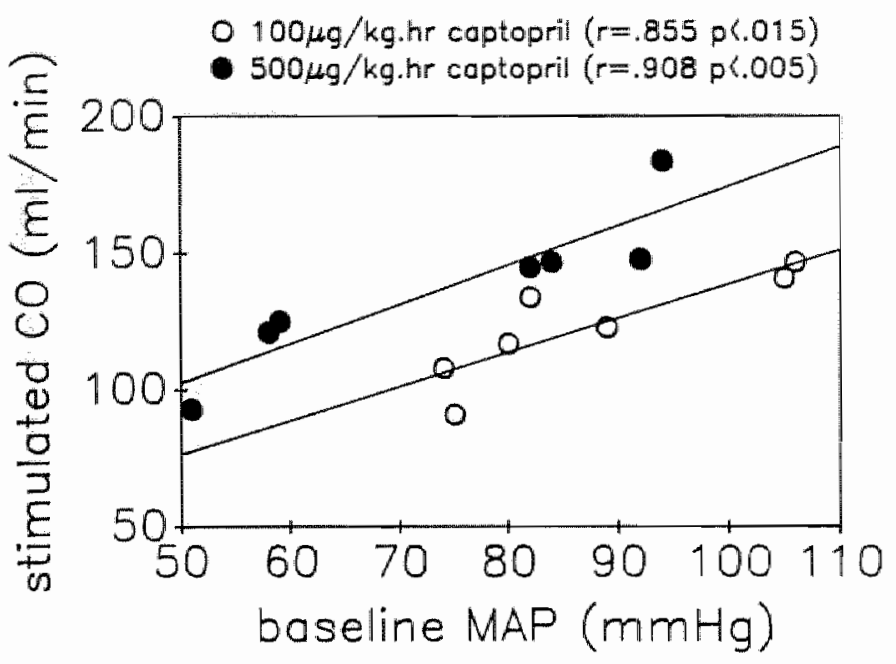

Fig. 7.5 Stimulated cardiac output related to baseline mean arterial pressure for the low and high dose captopril therapy from 3-5 weeks.

Table 7.3 Characterization of the experimental groups for captopril therapy from 1-21 days following ligation

Infarct Captopril

$\mathrm{n}$

Inf. size

BW

HW

HW/BW

resp.

LVEC
9

$40 \pm 3$

$310 \pm 9$

$1003 \pm 64$

$3.2 \pm 0.2$

$139 \pm 14$

$15.1 \pm 1.1$

\section{9}

$41 \pm 3$

$262 \pm 4^{*}$

$771 \pm 26^{*}$

$2.9 \pm 0.1$

$139 \pm 10$

$15.7 \pm 0.7$

For abbreviations and units, see table 7.1 


\subsubsection{Effects of captopril therapy during the healing period}

The two experimental groups are characterized in table 7.3. No rat died during therapy. Infarct sizes were similar for the 2 groups. In the captopril treated group, both body weight and heart weight were significantly decreased compared to non-treated animals. Although captopril decreased heart weight/body weight ratio, this did not reach significance. Captopril had no effect on respiration rate nor on ventricular dilatation (LVEC).

Verification of $A C E$ inhibition showed a depressed TPR response $(9.8 \pm 3.2 \%)$ to $150 \mathrm{ng} / \mathrm{kg}$ AngI. In non-treated infarcted rats, the response was $41 \pm 8 \%$.

Hemodynamics at baseline conditions are presented in table 7.4. Baseline $\mathrm{CO}$ did not differ between the 2 groups. However, in the captopril-treated group, this $\mathrm{CO}$ resulted from a significantly lower SV and a significantly higher HR, compared to the non-treated rats. SW was significantly decreased due to captopril therapy. Although captopril lowered MAP with $10 \%$, the effect was not found to be statistically significant.

Table 7.4 Baseline and stimulated hemodynamics after captopril therapy from 1-21 days following ligation (mean $\pm S E M$ )

\begin{tabular}{|c|c|c|c|c|}
\hline & \multicolumn{2}{|c|}{ Infarct control } & \multicolumn{2}{|c|}{$500 \mu \mathrm{g} / \mathrm{kg} \cdot \mathrm{hr}$ captopril } \\
\hline & Baseline & Stimulated & Baseline & Stimulated \\
\hline $\mathrm{dF} / \mathrm{dt}$ & $371 \pm 20$ & $452 \pm 23$ & $363 \pm 19$ & $438 \pm 29$ \\
\hline $\mathrm{PF}$ & $307 \pm 15$ & $361 \pm 17$ & $294 \pm 16$ & $353 \pm 23$ \\
\hline $\mathrm{CO}$ & $74 \pm 4$ & $111 \pm 5$ & $68 \pm 4$ & $104 \pm 7$ \\
\hline $\mathrm{HR}$ & $361 \pm 9$ & $397 \pm 6$ & $397 \pm 111^{*}$ & $417 \pm 10$ \\
\hline $5 V$ & $208 \pm 10$ & $281 \pm 13$ & $174 \pm 11^{*}$ & $249 \pm 16$ \\
\hline MAP & $90 \pm 3$ & $84 \pm 5$ & $82 \pm 5$ & $77+4$ \\
\hline $\mathrm{CVP}$ & $-0.2 \pm 0.3$ & $15.3 \pm 0.8$ & 1.11 .0 & $16.2 \pm 1.2$ \\
\hline TPR & $1.26 \pm 0.10$ & $0.66 \pm 0.05$ & $1.24 \pm 0.12$ & 0.6540 .06 \\
\hline os & $14.8 \pm 1.6$ & $13.1 \pm 1.1$ & $11.0 \pm 1.0$ & $11.3 \pm 0.9$ \\
\hline PEP & $41 \pm 2$ & $35 \pm 3$ & $38 \pm 1$ & $39+3$ \\
\hline $\mathrm{PEP} / \mathrm{LVET}$ & $0.49_{ \pm} 0.02$ & $0.41 \pm 0.03$ & $0.53 \pm 0.02$ & $0.52 \pm 0.03$ \\
\hline SW & $19 \pm 1$ & $21 \pm 2$ & $1441^{*}$ & $16+1^{*}$ \\
\hline C.I & $240 \pm 15$ & $361 \pm 19$ & $260 \pm 11$ & $395+21$ \\
\hline
\end{tabular}

\footnotetext{
"Significantly different from non-treated infarcted rats For abbreviations and units, see table 7.2
} 
Effects on cardiac performance are summarized in fig. 7.6. After stimulating the heart by volume loading, none of the measured parameters were altered by captopril the rapy (table 7.4) except for a decreased SW.

Thus, although with captopril therapy during the healing period cardiac performance was maintained, this can be attributed to a decreased SV and increased HR.

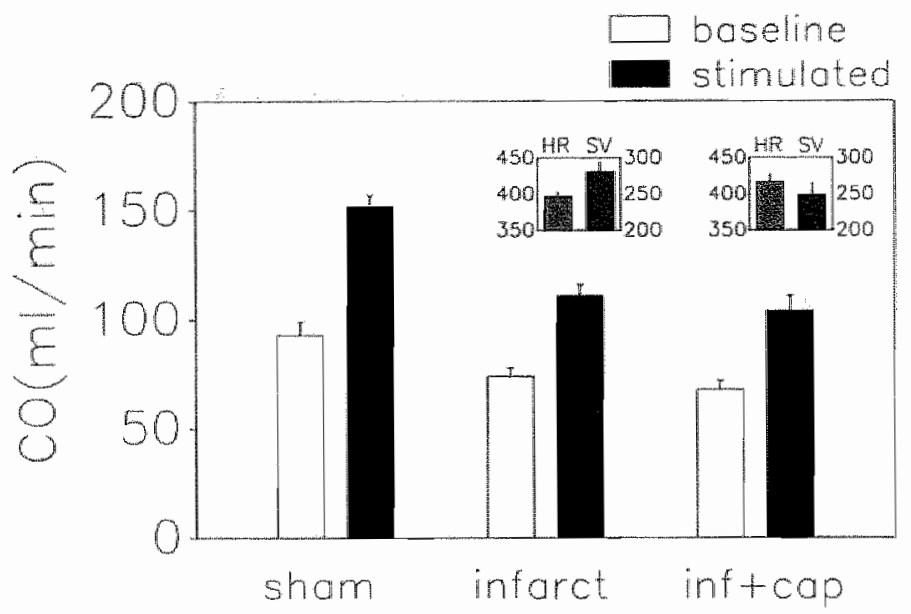

Fig. 7.6 Effects of captopril therapy from 1-21 days after infarction on cardiac performance and the contributions of HR (beats/min) and SV (mul) to stimulated CO. For comparison with non-infarcted rats, results from sham rats at 5 weeks are included.

\subsection{Discussion}

In the last decade, the use of ACE inhibitors in the therapy of heart failure has increased. Captopril, initially developed as an antihypertensive drug, has been reported to improve hemodynamics and decrease mortality in man (Franciosa, 1982; Guyatt, 1986; Packer, 1986) and in rats (Pfeffer et al, 1987) with heart failure.

The purpose of this study was three-fold: 1) to study acute effects of captopril in rats with heart failure; 2) to investigate effects of captopril therapy after the healing period from 21-35 days following infarction, and 3) to compare effects of captopril therapy cluring the healing period to effects of therapy after this period.

Acute captopril administration decreased MAP and TPR without effects on other parameters. Despite the reduction of afterload, no improvement of cardiac performance was found. This is in accordance with previous studies with acute captopril (Drexler et al, 1987a) and enalaprilate (Emmert et al, 1987).

In contrast to acute captopril treatment, short-term therapy from $3-5$ weeks resulted in both reduction of afterload and improved cardiac function. Moreover, 
while all hemodynamic effects of infarction had disappeared or tended to return towards sham values, except for MAP and TPR (which were reduced), indicators of mechanical properties of the heart were not significantly altered. Hemodynamic effects in the present study correspond well with results at comparable infarct sizes following 3 months of captopril treatment (Pfeffer et al, 1985a). In a study in which hemodynamic effects and effects on survival were studied in parallel (Pfeffer et al, 1987), hemodynamic improvement in rats with infarct sizes comparable to the ones in the present study was associated with increased survival.

Short-term captopril therapy from 1-21 days resulted in a 10\% reduction of MAP but no improvement of cardiac function. Moreover, the significantly decreased SV, together with the increased HR, indeed preserved $\mathrm{CO}$, but this indicates a detrimental state rather than improvement. After 3 months of captopril therapy no hemodynamic differences were reported when therapy was started either 2 or 21 days after infarction; data were therefore pooled (Pfeffer et al, 1985a). Results of the present study suggest that rats treated during the healing period (day 1-21) may have a worse hemodynamic start position for further therapy than non-treated rats.

Thus, acute captopril administration reduced afterload without improvement of cardiac function. Short-term captopril therapy, started when healing processes are considered to be complete, improved cardiac performance dose-dependently without adverse hemodynamic side-effects. This improvement could not be explained on the basis of decreased MAP. When short-term captopril therapy was given during the healing period, no hemodynamic improvement was found despite a (non-signifucant) MAP reduction. Thus, afterload reduction may be not the primary mechanism to improve cardiac function. Several other mechanisms may be involved.

Captopril has been suggested to be a preferential renal vasodilator (Smits et al, 1984; Drexler et al, 1987a). In rats after chronic infarction, decreased renal flow was specifically restored by acute captopril. (Drexler et al, 1987a). In contrast, diltiazem has a general vasodilating effect in the same model (Drexler et al, 1985). By specifically increasing renal blood flow, captopril could maintain or improve renal function at a lower perfusion pressure. The reduction of blood volume after chronic captopril, but not after hydralazine (Raya et al, 1988a) would support this suggestion. Preload reduction, reported after chronic (Pfeffer et al, 1985a, 1987), but not after acute captopril (Drexler et al, 1987a) suggests that effects on fluid balance may take too much time to cause a decreased preload after acute captopril. This long-term improvement in the absence of acute effects indicates that both effects should be studied to determine the therapeutic value of a drug. The renal vasodilating effect of captopril, however, does also not explain the different effects of therapy in the 2 different periods following infarction.

After infarction, the loss of contractile tissue and the consequent increase in preload evokes remodeling of the myocardium (Meerson, 1983; Gorlin, 1987). This remodeling process includes an increased number of myofibrils per myocyte; hypertrophy (Anwersa et al, 1986) and a reduced proportion of V1 myosin isoenzyme type fibers (Geenen et al, 1989) in addition to an increased connective tissue mass (Michel et al, 1988; Vracko et al, 1989). Chronic ACE inhibition can reverse these changes not only by reducing hypertrophy (Pfeffer et al, 1985a), but also by increasing $V 1$ proportion and normalization of connective tissue volume percentage (Michel et al, 1988). This results in hemodynamic improvement with decreased heart weight and reduced ventricular dilatation (Pfeffer et al, 1987). 
Studies in our laboratory have shown a transient increase in DNA synthesis during the healing period (Van Krimpen et al, 1988) which may be associated with the remodeling process. Captopril therapy during the healing period suppresses the increase in DNA synthesis and decreases heart weight (Van Krimpen et al, to be published), probably suggesting inhibition of the remodeling. Suppression of hypertrophy during the healing period results in a further increase in left ventricular dimensions (Fishbein et al, 1988). Decreased heart weight with preserved left ventricular endocardial circumference in the present study also indicates structural dilatation. Moreover, decreased SV ejected from a dilated left ventricle suggests reduced ejection fraction which is supported by an increased PEP/LVET ratio.

After the healing period, normalized CVP (chapter 4) and DNA synthesis (Van Krimpen et al, 1988) and no further changes in V1/V3 ratio (Geenen et al, 1989) indicates that responses may have become balanced and the heart is adapted to the altered physical condition. Captopril therapy started at this time clearly improves hemodynamics, suggesting that inhibition of further cardiac remodeling may be beneficial. Effects on cardiac remodeling as indicated by a reduction of heart weight and left ventricular dilatation were not found after 2 weeks captopril therapy, but could be shown after 3 months of captopril (Pfeffer et al, 1985a) and after 2 months of perindopril therapy (Michel et al, 1988). Results indicate that influences on cardiac remodeling may contribute to the hemodynamic effects of therapy. This could be due to direct or indirect effects.

Regression of myocardial hypertrophy after captopril is also known in antibypertensive therapy (Sen, 1982) and could be an indirect effect of afterload reduction. However, not all antihypertensives decreased hypertrophy (Sen, 1982), indicating no direct relationship. An increased preload due to specific arterial vasodilatation could explain the lack of effect on hypertrophy (Tsoporis et $\mathrm{al}_{*} 1989$ ).

Captopril could influence hypertrophy directly since it not only inhibits circulating $A C E$, but also the myocardial intracellular renin-angiotensin system ( $R e, 1987$; Dzau et al, 1987, Lindpaintner et al, 1988). Intracellular angiotensin II is suggested to have mitogenic activity by its interaction with intracellular nuclear binding sites (Dzau et al, 1987) and can be viewed as a locally active growth factor ( $R e$ et al, 1988).

Furthermore, captopril could influence hypertrophic processes indirectly by its interaction with the sympathetic nervous system, since angiotensin II facilitates noradrenaline release during sympathetic stimulation. Noradrenaline has been shown to stimulate hypertrophy in cultured rat myocytes through alpha-receptor stimulation (Simpson, 1988) whereas beta-adrenergic stimulation causes hypertrophy (Jeppson et al, 1986) and a shift to V3 fibers (Rupp et al, 1987). Beta-blockade during the healing period in infarcted rats reduces hypertrophy (Fishbein et al, 1988).

The proposed cellular effects of $\mathrm{ACE}$ inhibition may not be restricted to the myocytes since the increased volume percentage of collagen after infarction was normalized by perindopril (Michel et al, 1988) and chamber stiffness was normalized by captopril (Pfeffer et al 1985a).

in conclusion, whereas acute captopril treatment has no effect on cardiac function, short-term therapy improves cardiac function dose-dependently if treatment is started 3 weeks after infarction. No improvement was found after captopril therapy during the first 3 weeks after infarction. Reduction of preload and afterload as well as a direct effect on cardiac remodeling can contribute to the effects of captopril therapy. Results indicate that adaptive processes like cardiac remodeling should take 
place before captopril therapy exerts beneficial effects. The hemodynamic state after early therapy, tachycardia and decreased SV suggests that the moment of starting captopril therapy should be chosen carefully. 


\section{BENAZEPRIL IN HEART FAILURE}

\subsection{Introduction}

During the last decade, the use of angiotensin $\mathbb{1}$ converting enzyme (ACE) inhibitors in the therapy of heart failure has increased. ACE inhibitors not only improve hemodynamics, but can also reduce mortality (Cohn et al, 1987; Consensus trial, 1987).

Since captopril, which was the first clinically used drug of this type of agents, many new ACE inhibitors have been developed. Different classes of ACE inhibitors have been introduced for clinical use, e.g. sulfhydryl containing inhibitors like captopril, carboxylalkyl inhibitors like enalapril and phosphorus containing inhibitors like fosenopril (Petrillo et al, 1987; Ondetti, 1988). All three classes bind to the active site of the enzyme in a similar manner (Petrillo et al, 1987), but structure related differences has been reported in tissue distribution and routes of elimination (Ondetti, 1988). These could contribute to differences in pharmucological actions.

In the previous chapter, captopril therapy from 3.5 weeks following infarction has been shown to improwe cardiac performance in rats. Besides inhibition of circulating $\mathrm{ACE}$, different mechanisms of action were discussed including neurohumoral, hemodynamic and biochemical mechanisms. In order to investigate whether the effects of captopril are restricted to SH containing $\mathrm{ACE}$ inhibitors, and to elucidate the possible mechanisms of action of $A C E$ inhibitors in the therapy of heart failure, in the present study a newly developed $\mathrm{ACE}$ inhibitor from the class of carboxylalkyl type inhibitors, benazepril (CGS 14824A) was investigated.

The aim of the study was to investigate hemodynamic effects of benazepril therapy from 3 to 5 weeks following infarction and to compare these effects to those obtained with captopril in the previous chapter. 


\subsection{Experimental protocol}

\subsubsection{Dose finding study}

A dose range finding study was performed in normal Wistar rats to obtain information on the amounts of benazepril and captopril to be infused to achieve comparable blockade of converting enzyme. Groups of 3-4 male Wistar rats (body weight approximately 300 grams) were subcutanously implanted with an osmotic minipump (Alzet 2001, Palo Alto, USA), containing saline, captopril in concentrations to deliver 100 or $500 \mu \mathrm{g} / \mathrm{kg}$.hr or benazepril in concentrations to deliver 3,30 or $300 \mu \mathrm{g} / \mathrm{kg} / \mathrm{hr}$. Treatment lasted four days. Then, after induction of pentobarbital anesthesia $(60 \mathrm{mg} / \mathrm{kg}$ i.p.) catheters were inserted into the left femoral vein for injections and the left femoral artery for blood pressure recording. Body temperature was maintained at $37^{\circ} \mathrm{C}$. After a stabilization period of 30 minutes, animals were injected with angiotensin I in a dose range of $10-30.000 \mathrm{ng} / \mathrm{kg}$ and changes in mean arterial pressure were recorded. Injections were given in random order.

From the average changes in MAP, dose-response curves were constructed. From these, 3 doses of benazepril were chosen to result in a degree of ACE inhibition comparable or greater than achieved by 100 and $500 \mu \mathrm{g} / \mathrm{kg}$.hr captopril, respectively.

\subsubsection{Hemodynamic study}

\section{Surgery protocol}

Male Wistar rats, weighing $250-350$ gram at the start of the protocol were subjected to coronary artery ligation as described in section 2.2.1. Infarcts were allowed to heal. Three weeks after ligation, under light ether anesthesia, osmotic minipumps filled with benazepril solution, were implanted subcutaneously between the shoulder blades. Benazepril was given at 20,100 and $500 \mu \mathrm{g} / \mathrm{kg}$.hr. After one week rats were anesthetized with pentobarbital and electromagnetic flow probes were implanted. At the same time pumps were replaced. After 5-6 days recovery catheters were implanted for MAP and CVP measurements and for infusions. In addition, ECG electrodes were implanted (cf. section 2.4.1-2.4.5). Experiments were started at least one day following this surgery.

\section{Mensuring and therapy protocol}

Hemodynamic measurements were performed after two weeks therapy. Rats were connected to the measuring equipment and signals were fed into preprocessor and computer for on-line derivation of hemodynamic parameters. After 45-60 min equilibration baseline values were obtained and $A C E$ inhibition was verified by measuring TPR responses to $150 \mathrm{ng} / \mathrm{kg}$ Ang I. When hemodynamics had returned to pre-Ang I values, CO was maximally stimulated by volume loading (cf. 2.5). Hemodynamics were monitored till CVP had returned to its baseline value or maximally 60 
min after volume load. Data were stored on a hard disk for later analysis. After the experiments rats were killed to determine infarct sizes (cf. 2.3).

\subsection{Results}

\subsubsection{Dose finding study}

Dose-response curves for blood pressure responses to bolus injections of angiotensin I are presented in figure 7.7. Captopril and benazepril caused a dose-dependent shift of the dose response curve to the right. From these curves, doses of 20,100 and $500 \mu \mathrm{g} / \mathrm{kg}$.hr benazepril were selected to encompass a similar range of ACE inhibition, compared to 100 and $500 \mu \mathrm{g} / \mathrm{kg} . \mathrm{hr}$ captopril. "These doses were used in the study of 2 weeks therapy.

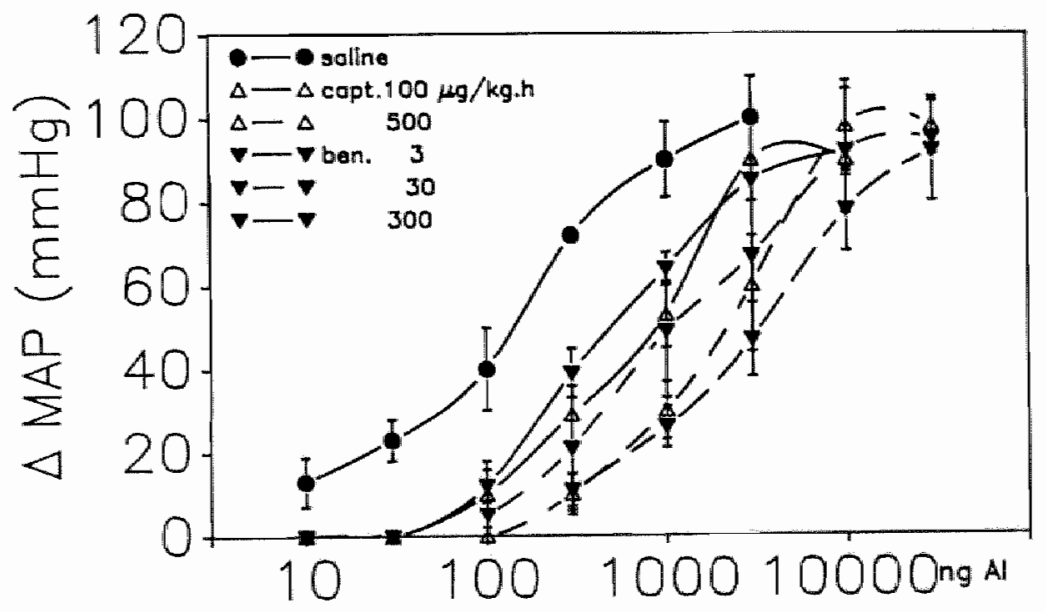

Fig. 7.7 Effects of 4 days captopril and benazepril pretreatment on the dose-response curves for the blood pressure response to angiotensin I in normal Wistar rats.

\subsubsection{Hemodynamic study}

\section{General}

Acute mortality caused by coronary artery ligation is described in chapter 4 . Ten rats died during treatment with $20 \mu \mathrm{g} / \mathrm{kg}$.hr benazepril, 4 rats during $100 \mu \mathrm{g} / \mathrm{kg} . \mathrm{hr}$ and 1 during $500 \mu \mathrm{g} / \mathrm{kg}$.hr. In 3 rats of the last group, there was evidence for peripheral circulation disturbance as judged from a discoloration of the cannulated 
hindleg. After exclusion of data from rats with infarcts $<21 \%$, groups (characterized in table 7.5) had comparable infarct sizes.

In infarcted rats, BW was significantly lower than in sham animals but no differences due to therapy were found. Benazepril decreased heart weight. However, the decrease of the ratio of heart weight to body weight was not statistically significant. Tachypneu and left ventricular dilatation were not significantly changed by benazepril therapy.

ACE inhibition measured as TPR response to a submaximal dose of $150 \mathrm{ng} / \mathrm{kg}$ Ang I resulted for sham controls and infarct controls in a TPR increase of $41 \pm 17$ and $41 \pm 8 \%$ respectively. In the treated groups this response was significantly reduced (benazepril low $22 \pm 7$, medium $17 \pm 5$ and high dose $6 \pm 2 \%$ ) indicating long-term ACE inhibition.

Table 7.5 Characterization of experimental groups for benazepril therapy from 3-5 weeks after ligation

sham

\section{7}

ก.

inf. size (\%)

$\mathrm{BW}(\mathrm{g})$

0

$\mathrm{HW}$ (mg)

$\mathrm{HW} / \mathrm{BW}(\mathrm{mg} / \mathrm{g})$

resp $\left(\mathrm{min}^{-1}\right)$

LVEC $(\mathrm{mm})$ infarct

benazepril benazepril benazepril

$20 \mu \mathrm{g} / \mathrm{kg} \cdot \mathrm{hr} 100 \mu \mathrm{g} / \mathrm{kg} \cdot \mathrm{hr} 500 \mu \mathrm{g} / \mathrm{kg} \cdot \mathrm{hr}$

Abbreviations: Inf. size: infarct size; BW: body weight; HW: heart weight; resp.: respiration rats; LVEC: mean left ventricular endocardial circumference.

* Significantly different from sham operated rats

*ignificantly different from non-treated infarcted rats

\section{Hemodynamic effects}

Hemodynamic effects of 5 weeks infarction are described in detail in chapter 4. When small infarcts were excluded, depression of cardiac performance was significantly present even at baseline conditions.

Benazepril dose dependently decreased MAP without significant effects on HR (fig. 7.8). None of the flow related parameters were altered by benazepril nor was CVP. Indicators of mechanical properties of the heart, PEP and SW, were not affected. SW remained significantly lower compared to sham rats. Although PEP/LVET was decreased at the highest dose, this effect was not statistically significant. QS was significantly decreased at the highest dose (table 7.6). Despite the reduction in MAP, CO was not increased (fig. 7.9). 

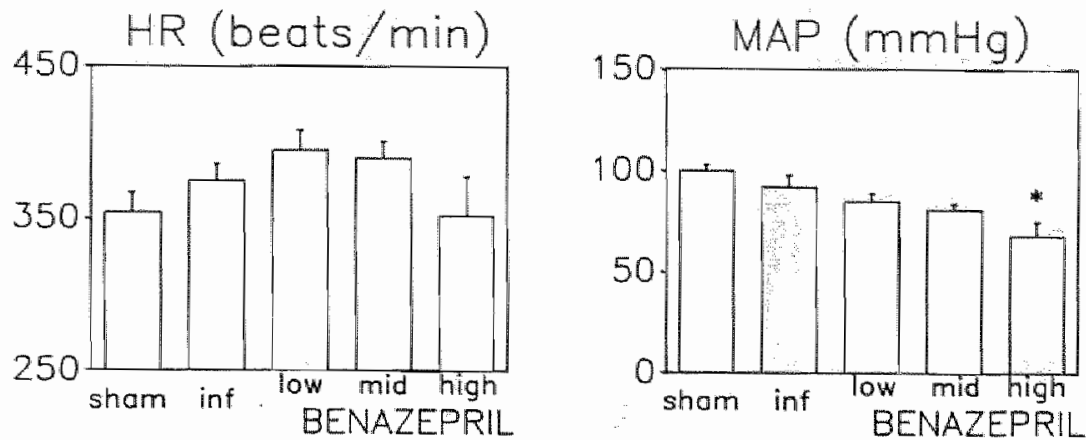

Fig. 7.8 Effects of benazepril therapy from 3-5 weeks after ligation on baseline HR and MAP.

Table 7.6 Baseline hemodynamics for the experimental groups

sham

\section{benazepril}

benazepril

$100 \mu \mathrm{g} / \mathrm{kg} \cdot \mathrm{hr}$ benazepri1

$500 \mu \mathrm{g} / \mathrm{kg} \cdot \mathrm{hr}$

$\begin{array}{lccccc}\mathrm{n} & 7 & 8 & 7 & 9 & 8 \\ \mathrm{dF} / \mathrm{dt} \mathrm{(mL/ \textrm {s } ^ { 2 } )} & 421 \pm 23 & 339 \pm 30 & 322 \pm 38 & 373 \pm 32 & 363 \pm 51 \\ \mathrm{PF}(\mathrm{ml} / \mathrm{min}) & 366 \pm 19 & 285 \pm 24^{+} & 275 \pm 31 & 311_{ \pm 32} & 307 \pm 39 \\ \mathrm{SV}(\mu 1) & 257 \pm 16 & 199_{ \pm} 17^{*} & 178 \pm 18 & 205 \pm 20 & 216 \pm 25\end{array}$

TPR (mnitg)

$\mathrm{min} / \mathrm{ml}$ )

PEP (ms)

PEP/LVET

SW $(m \mathrm{mHg} \cdot \mathrm{m} 1)$

os (nas)
$1.12 \pm 0.10 \quad 1.25 \pm 0.15 \quad 1.32 \pm 0.19$

$36 \pm 1$

$40 \pm 1^{*}$

$39 \pm 2$

$0.49 \pm 0.01$

$0.54 \pm 0.02^{4+}$

$0.55 \pm 0.03$

$15 \pm 2$

$14.5 \pm 0.9$

$1.18 \pm 0.19$

$38 \pm 2$

$0.53 \pm 0.03$

$17+2$

$16.7 \pm 0.9 \quad 17.0 \pm 1.9$

$15.0 \pm 1.1$

1. $00 \pm 0.14$

$37 \pm 2$

$0.46 \pm 0.03$

$15 \pm 3$

$12 \cdot 2 \pm 1.2^{*}$

Abbreviations: dF/dt: aortic flow acceleration; PF: peak flow; SV: stroke volume; TPR: total peripheral resistance; PEP: pre-ejection period; LVEI: left ventricular ejection time; sU: stroke work; OS: duration of the OS interval

For significances, see table 7.5 


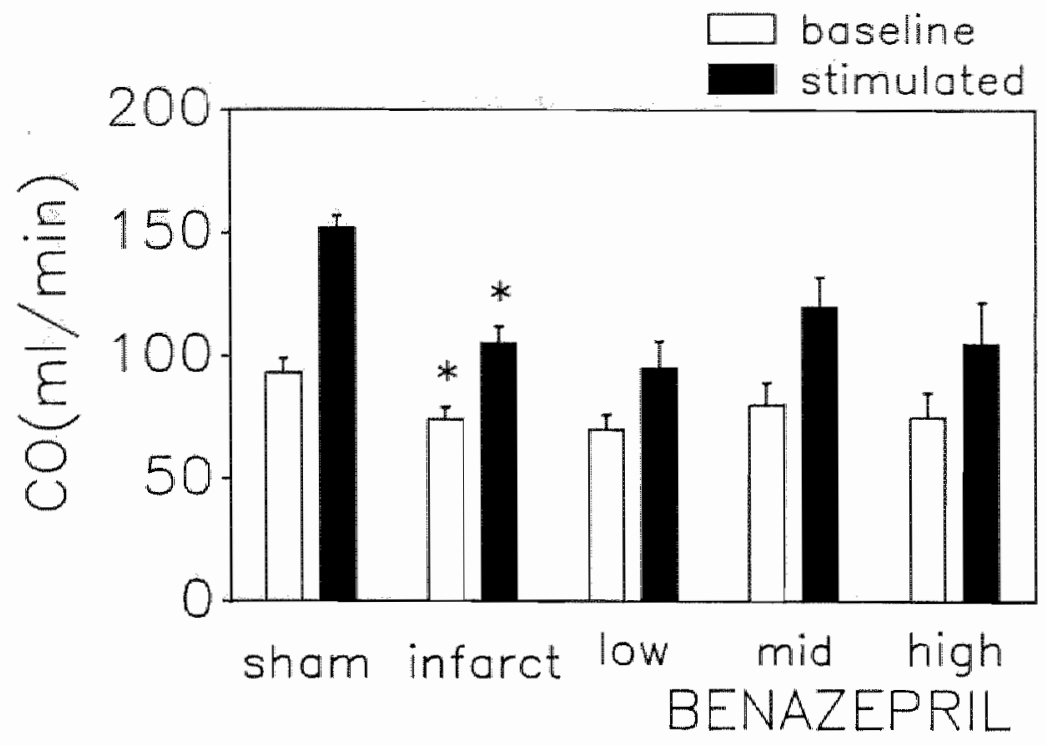

Fig. 7.9 Effects of benazepril therapy from 3.5 weeks after ligation on cardiac performance.

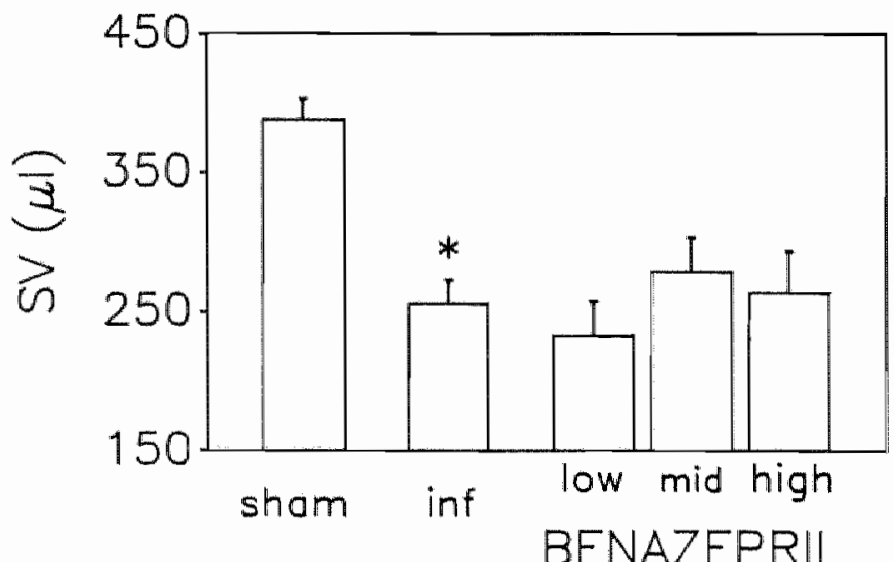

Fig. 7.10 Contribution of $S V$ to stimulated $C O$ after 2 weeks benazepril therapy. 
Effects on cardiac performance after volume loading are shown in fig. 7.9. The dose-effect relationship was bell-shaped. A slight improvement was found for the medium dose. Effects on peak CO are reflected in effects on SV (fig. 7.10), whereas SW was not increased. Stimulated PEP $(32 \pm 1 \mathrm{ml})$ and PEP/LVET $(0.38 \pm 0.03)$ at the highest dose of benazepril were significantly decreased; compared to non-treated infarcted rats (PEP: $38 \pm 2 \mathrm{~ms}$; PEP/LVET: 0.48 \pm 0.02 ), which was probably due to decreased MAP $(59 \pm 9$ compared to $93 \pm 6 \mathrm{mmHg}$ ) rather than to increased contractility and ejection fraction, respectively.

Thus, whereas 2 weeks benazepril significantly inhibited circulating ACE, measured as depressed TPR response to ang $I$, and reduced MAP cardiac performance was not significantly improved.

\subsection{Discussion}

During the 12 years, since the first publication on captopril (Ondetti et al, 1977), a great number of potent ACE inhibitors have been developed. Most of the properties of these drugs are related to their common specific mechanism of action: ACE inhibition. Drugs are divided into different classes according to their structure; sulfhydryl type, carboxyl type and phosphorus type (Ondetti, 1988). These structural differences possibly contribute to differences in pharmacological actions due to different tissue distribution and/or routes of elimination (Ondetti, 1988). Benazepril is a newly developed carboxyl type of ACE inhibitor (Schaller et al, 1985; Waeber et $\mathrm{al}_{1}$ 1987).

Based on MAP responses to ang I injections after 4 days of treatment in normal Wistar rats, doses of 20,100 and $500 \mu \mathrm{g} / \mathrm{kg}$.hr benazepril were chosen to encompass the range of ACE inhibition after the 100 and $500 \mu \mathrm{g} / \mathrm{kg}$.hr captopril (chapter 7A). After 2 weeks of benazepril therapy in rats with heart failure, ACE activity as measured from TPR responses to ang I was reduced to a similar degree or more compared to captopril (chapter 7A).

Also the degree of MAP reduction after 2 weeks of benazepril was comparable to that after captopril (chapter 7A). However, despite these similarities to captopril treatment, no significant improvement of cardiac performance was found after 2 weeks benazepril therapy. This implies that neither long-term inhibition of circulating ACE nor lowering of MAP per se leads to improvement of cardiac performance. Other mechanisms, different for benazepril compared to captopril, should be involved. For captopril the following mechanisms of action have been suggested (chapter $7 \mathrm{~A}$ ):

- a specific renal vasodilatation which preserves or augments renal function while perfusion pressure is reduced. This could contribute to preload reduction.

- intervention of cardiac remodeling, resulting in regression of hypertrophy, increased $V 1$ myosin isoenzyme proportion and reduction of connective tissue mass. This could be a direct effect of inhibition of local renin-angiotensin systems (Lindpaintner et al, 1988) or an indirect effect via the sympathetic nervous system (Simpson, 1988; Jeppson et al, 1986).

Captopril has been suggested to be a preferential renal vasodilator (Smits et al, 1984; Drexler et al, 1987a). No structure-related differences in renal ACE inhibition 
were found (Cushman et al, 1989). Increased circulating prostaglandin levels, found after captopril (Nishimura et $\mathrm{al}_{\text {, }}$ 1987), but not after enalapril (Williams, 1988), could contribute to the renal effects since they can be attenuated by indomethacin (Nishimura et al, 1987; Packer, 1988). Also differences in interaction with the sympathetic nervous system have been suggested to attribute to prostaglandins (Van Gilst et al, 1986).

Both captopril and zofenopril (sulfhydryl) are potent inhibitors of cardiac ACE whereas enalapril and ramipril (non-sulfydryl type inhibitors) cause only slight inhibition (Cushman et al, 1989) suggesting that the presence of an SH group may be important for cardiac effects. In the present study, however, benazepril significantly decreased heart weight. Also perindopril, a non-sulfhydryl ACE inhibitor, significantly reduced heart weight after 2 months of therapy (Michel et al, 1988) suggesting also cardliac effects of ACE inhibitors without SH group. In the present study, a decrease in heart weight, passibly indicating regression of hypertrophy, not necessarily results in improvement of cardiac performance. Moreover, reduced mortality in infarcted rats was found after captopril (Pfeffer et al, 1987), but also after enalapril (Sweet et al, 1987). This suggests that effects on survival are neither depending on the presence of SH group nor on the potency of cardiac ACE inhibition.

Captopril and zofenopril are potent brain ACE inhibitors compared to enalapril and ramipril (Cushman et al, 1989). However, also benazepril has a significant higher brain ACE inhibitory activity compared to enalapril (Zimmerman et al, 1987). Selective brain ACE may play a role in the central regulation of the cardiovascular system (Cushman et al, 1989).

In conclusion, 2 weeks therapy with benazepril did not lead to significant improvement of cardiac performance. Since circulating ACE inhibition and reduction of MAP after benazepril were comparable to the effects after two weeks captopril, these do not seem to be the primary mechanisms to improve cardiac function. Other mechanisms, possibly related to structural differences may play a role as well. 
chapter 8

\section{CONCLUDING REMARKS AND OUTLOOK}

\subsection{General}

Congestive heart failure is a common clinical manifestation of different heart diseases rather than a disease per se. The two major systems involved in heart failure, the heart and the peripheral circulatory system, can contribute to both compensation and decompensation (Ruggie, 1986; Remme, 1986). Treatment has traditionally been directed towards reduction of symptoms of congestion, which may: be the result of the peripherall compensatory mechanisms rather than the effect of the cardiac dysfunction itself (Cohn, 1988). Considerable advances have been made with regard to the relief of symptoms. This, however, did not result in significantly improved survival (Cohn, 1988). If the course of the cardiac alterations leading to failure could be affected as well, it might provide a new opportunity for therapy. That implies the need for a better understanding of the pathophysiology and the effects of ireatment and, therefore, the need for a suitable animal model. For practical reasons this should be a small animal model.

\subsection{Models, pathophysiology and therapy}

The purpose of this thesis was to study pathophysiology and therapy of heart failure in a sultable rat model. Two possible models, based on the major conditions leading to heart failure in man, were explored, i.e. chronic hypertension and myocardial infarction. The cardiac function curve was used in the conscious rat to measure cardiac performance not only at rest, but also at an extra demand, as discussed in chapter 2. The model of the spontaneously bypertensive rat did not prove to be a practical model, because of the high mortality. Also a state of high output failure could not be excluded (chapter 3). Therefore, further studies were performed in the model of myocardial infarcted rats. Effects of acute and chronic treatment with 3 types of agents, milrinone, dobutamine and captopril, which are known to improve hemodynamics in man (Baim, 1989, Thomas et al, 1987; Sharpe, 1989), were studied in this model. Acute effects differed from long-term effects for all three drugs. Although improvements of cardiac performance after long-term therapy were 
comparable, the underlying hemodynamic effects were different and might be associated with different cardiac effects. Also the different hemodynamic effects of treatment during different periods following infarction, as is shown in the early and later captopril therapy studies (chapter 7A), might attribute to different cardiac effects.

\subsubsection{Early captopril}

In the first 3 weeks after myocardial infarction, the initially changed hemodynamic parameters (CVP, MAP, CO and SV) are normalized (chapter 4). During this period healing processes and cardiac remodeling have been described, resulting in scar formation (Pfeffer et al, 1979a), hypertrophy of the spared myocardium (Anversa et al, 1986) and an increase in myocardial connective tissue (Vracko et al, 1989; Jalil et al, 1989). Experiments in our laboratories have shown a transient increase in DNA synthesis in the heart (Van Krimpen et al, 1988), which could be associated with these cardiac processes. Altered mechanical properties of the heart, left ventricular dilatation and depressed cardiac performance at 3 weeks after infarction (chapter 4) may represent the result of the cardiac remodeling. The healing period in infarcted rats corresponds well with the first stage in the development of heart failure described by Gorlin (1987) and Katz (1989), in which the heart adapts to the altered physical load.

Captopril therapy during the healing period preserved cardiac function compared to non-treated rats (chapter 7A). The increased heart rate and decreased stroke volume, however, indicate a shift to an energetically less favourable state. This is supported by the enhanced wall stress due to structural dilatation (Vogt et al, 1987a), as indicated by reduced heart weight with preserved left ventricular dimensions. In addition, reduced stroke volume ejected from the same left ventricular cavity indicates a decreased ejection fraction which is also suggested by the increased PEP/ LVET ratio. Results indicate that captopril treatment during the healing period has unfavorable effects, probably due to an interaction with cardiac remodeling processes. The inhibitory effects of captopril on the increased DNA synthesis following infarction (van Krimpen et al, to be published) supports this suggestion.

Results indicate that the cardiac remodeling during the healing period can be regarded as a functional adaptation.

\subsubsection{Later captopril}

Captopril therapy started after this healing period turned out to be beneficial. The period of treatment, 3-5 weeks after infarction, is characterized by normal baseline hemodynamics and DNA synthesis (van Krimpen et al, 1988) and no further changes in the left ventricular dilatation or myosin isoenzyme shift (Geenen et al, 1989). Responses may have become balanced and failure has entered a "compensated" stage (Meerson, 1983; Katz, 1989). However, elevated plasma ANP, reduced contractility, left ventricular dilatation and tachypnea indicate cardiac dysfunction which is confirmed by a depressed cardiac performance during volume load (chapter 4). 
Captopril therapy restores the depressed cardiac function without increasing heart rate or stroke work (chapter 7A). The study with benazepril (chapter 7B) indicates that although inhibition of circulating $\mathrm{ACE}$ and lowering of blood pressure may have contributed to the effects, these are not the primary mechanisms to improve cardiac function. Other mechanisms, including different effects on cardiac remodeling and reduction of left ventricular preload (Tsoporis et al, 1989) may have contributed as well.

Although after the rather short ( 2 weeks) period of treatment, significant effects on cardiac remodeling could not be shown, longer therapy with captopril (Pfeffer et al, 1985a) and perindopril (Michel et al, 1988) support the possibility of their contribution. Reduction of hypertrophy, attenuation of left ventricular dilatation, a shift to myosin isoenzyme V1 type fibers and reduction of interstitial tissue mass have been reported. The reduced preload found by Pfeffer et al (1985a) may be reflected. in the decreased ANP levels found by Michel et al (1988). The importance of the effects of ACE inhibition is supported by an increased survival with captopril (Pfeffer et al, 1985b) and with enalapril in rats (Sweet et al, 1987).

Comparison of the results of early and later captopril therapy, however, indicates that timing and type of therapeutic intervention in cardiac remodeling should be chosen carefully.

\subsubsection{Dobutamine}

Whereas the contribution of effects on cardiac remodeling with the later captopril therapy are mostly hypothetical, the study with dobutamine provides additional evidence for structural cardiac changes and its consequences. In non-treated infarcted rats the inotropic response to acute dobutamine was reduced. It is suggested that this common phenomenon of impaired contractile response may not be caused by receptor down-regulation (Clozel et al, 1987; Vleeming et al, 1989) but may be related to post-membrane processes (Takeda et al, 1987). In chapter 6 it is discussed that a shift from V1 to V3 type fibers may be responsible for this phenomenon and, moreover, that this shift could be reversed by long-term intermittent dobutamine (Buttrick et al, 1988). This explains not only the improved cardiac performance without circulating dobutamine, but also the restored inotropic response to acute dobutamine.

In contrast to hypertrophy with a shift to $V 3$ fibers, hypertrophy with at shift to V1 fibers as occurs after physical training or thyroid hormones (Schever et al, 1987), is not associated with increased cell death (Buttrick et al, 1988) nor with reduced capillary density (Anversa et al, 1987; Ecker et al, 1989). A shift towards V1 fibers therefore could be a direct effect of the drug but may also reflect the myosin gene expression in a state of better energy supply. Nevertheless, an increased V1 proportion may indicate a better cardiac condition. Whereas both captoprill and dobutamine could cause such a shift, mechanisms may be different, since dobutamine, but not captopril, increased contractility and stroke work. Preload (Pfeffer et al, 1985a) and afterload reduction with captopril, but not with dobutamine (Buttrick et al, 1988), could be responsible for this difference. 


\subsubsection{Milrinone}

In contrast to captopril and dobutamine, millrinone is not known to influence cardiac remodeling. The inotropic response to acute cAMP stimulation also depends on the percentage $V 1$ type fibers (Horowits et al, 1987). The inotropic response to milrinone was found to be similar before and after 7-12 days milrinone treatment (Emmert et al, 1987) indicating no effect on V1 proportion. If the V1 proportion reflects cardiac condition, then the pronounced increase in cardiac contractility after 2 weeks milrinone treatment without a shift to V1, indeed improves cardiac performance but in the end may lead to increased cell death.

\subsection{Future outlook}

From the present thesis it can be concluded that improvement of cardiac performance following myocardial infarction in rats is possible with different type of agents. The improvement of cardiac performance in rats may mirror the symptomatic relief in patients. However, symptoms may be the result of peripheral compensatory mechanisms rather than an effect of the cardiac disease itself. The cardiac remodeling could also contribute to the evolution of heart failure and intervention may delay progression of the cardiac disease. Favorable effects of intervention in cardiac remodeling in rats may be reflected in a restored V1 myosin isoenzyme proportion. Although myosin shifts due to cardiac overload in man occur in the light chain rather than in the heavy chain (Schaub et al, 1987; Hoffmann et al, 1987) mechanical results are similar (Bouvagnet et al, 1989; Morano et al, 1988). The observation that the effects of captopril on cardiac remodeling after infarction in man paralleled that in rats (Pfeffer et al, 1987, 1988; Sharpe et al, 1988) supports the hypothesis. Moreover, enalapril therapy increases survival in man (Consensus Trial, 1987).

Thus, the ultimate target for therapy of heart failure should be relief of symptoms to improve quality of life and simultaneous intervention in cardiac remodeling to delay progression of the disease and increase life expectancy.

A distinction should be made between favorable (1-3 weeks) and unfavorable (3-5 weeks) remodeling, since the former may be a necessary physiological response to the primary myocardial damage. In order to choose and time treatment rationally, more should be learned about the mechanisms involved in the remodeling processes, from the level of the whole system to the cellular level. The presented model of conscious myocardial infarcted rats can contribute to the research in this field and provides a suitable model to test future therapeutic agents. 


\section{LIST OF REFERENCES}

Abelmann WH, Wagner RL, Bajusz E. Serial hemodynamic observations in hereditary cardiomyopathy of the syrian hamster. Recent Adw Stud Cardiac Struct Melab 21, 509-517, 1973.

Alonso DR, Scheidt $S$, Post $M$, Killip $T$. Pathophysiology of cardiogenic shock. Quantification of myocardial necrosis: clincial pathologic and clectrocardiographic cortelations. Circulation 48 , 588-597, 1983.

Alousi AA, Grant AM, Allen PD, Pagani ED. Effects of milrinone on Ca * sensitivity of myofibrillar $\mathrm{Mg}$-adenosine triphosphatase isolated from normal human and canine hearts. J Pharmacol Exp Ther $246,30-37,1988$.

Anwersa P. Loud AV, Lewicky V, Guideri G. Left ventricular failure induced by myocardal infaration I myocyte hypertrophy. Am J Physiol 248, H876-H882, 1985.

Anversa $P$, Beghi $C$, Kikkawa $Y$, Olivetti $G$. Myocardial infarction in rats, infaret size, myocyte hypertrophy and capillary growth. Circ Res 58, 26-37, 1986.

Anversa $P$, Ricci $R$, Olivetti $G$. Effects of exercise on the capillary vasculature of the rat heatt. Circulattion 75 (suppl 1), 12-18, 1987.

Applefeld MM et al. Intermittent, continuous outpatient dobutamine infusion in the management of congestive heart fallure. Am J Cardiol $51,455-458,1983$.

Baim DS, McDowell AV, Cherniles J, Monrad ES, Parker JA, Edelson J, Braunwald E, Grossman W. Evaluation of a new bipyridine inotropic agent - milrinone - in patients with severe congestive heart failure. New Engl J Med 309, 748-756, 1983.

Baim DS, Monrad ES, McDowell AV, Smith H, Lanoue A, Braunwald E, Grossman W. Milininonc therapy in patients with severe congestive heart failure: initial hemodynamic and clinical observations. In: Milrinone: investigation of new inotropic therapy for congestive heart failure (eds: Braunwald E et al). Raven Press, New York, 1984, pp 143-153.

Baim DS, Colucci WS, Monrad ES, Smith HS, Wright RF, Lanoue A, Gauthier DF, Ransil BJ, Gross. man W, Braumwald $E$. Survival of patients with severe congestive hoart failure treated with oral milrinone. I An Coll Cardial 7, 661-670, 1986.

Baim DS. Effect of phosphodiesterase inhibition on myocardial oxygen consumption and coronary bloot flow. Am J Cardiol 63, 23A-26A, 1989.

Bayliss J, Norell M, Canepa-Anson R, Sution G, Poole-Wilsom P. Untreated heart failure: clinical atud neura-endocrine effects of introducing diuretics. Br Heart J 57, 17-22, 1987.

Berger M. MCSherry CK. Outpaticnt dobutame infusion ming a totally implantable infusion punp lor refractory congestive heart faiture. Chest $88,295-296,1985$.

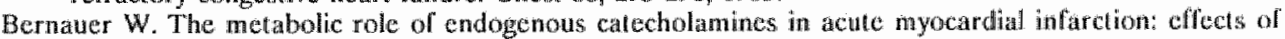
reserpinization and of infused noradirenaline. Eur J Pharmacol $88,161-168,1983$.

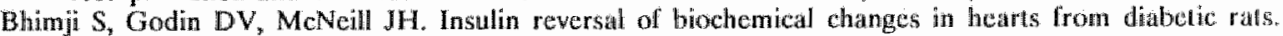
An $J$ Physiol 251, $1670-4675,1986$.

Böm M, Diet F, Feiler G, Kemkes B, Erdmann E. Alpha-adrenoceptors and alphatronoceplor mediated positive inotropic effects in failing human myocardium. I Caraliovase Pharmacol 12 , $357.364,1988$

Böm M, Schnabel P, Erdmann E. Increase of a $40 \mathrm{kDA}$ perlussis toxin substrate accompanics reduced CAMP formation and inotropic responsiveness in the failing human heart in dilated but not in ischemic cardiomyopathy. Nawnyn Schmiedeberg"s Arch Pharmacol (suppl) 339, R252, 1989.

Botting JH, Johnston KM, MacLeod BA, Walker MJA. The eflect of modification of sympathatic activity on responses to ligatyion of a coronary artery in the conscious $t$ ast. $\mathrm{Br} \mathrm{J}$ Phathacol $79,265-271$. 1983.

Bouvagnet $P$, Märhofer H, Leger JOC, Puech $P$, Leger JJ. Distribution pallern of alpha* and beta-myosin in normal and diseased human wentricular myocardium. Basic Res Cardiol $84,91-102$, 1989 .

Braunwald E. Newer postive inotropic agents. Circulation 73 (suppt 3), 237-239, 1986 


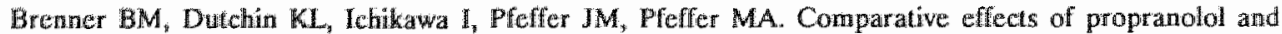
madolol on renal blood flow in normal rats afud rats with congestive beart failure. Am Heart J 108, $1144-1147,1984$.

Bristow MR, Ginsburg $\mathbb{R}$, Minobe $W_{1}$ Cubiccioti $\mathbb{R S}$, Sageman WS, Lurie $K$, Billingham ME, Hartison DC, Stinson $\mathrm{EB}$. Decreased catecholamine sensitivity and beta-adrenergüc receptor density in failing human herittai New Engl J Med 307, 205-211, 1982.

Bristow MR, Ginsburg R, Umans $V$, Fowler $M$, Minobe W, Rasmussen $R$, Zera $P$, Menlowe $R$, Shah $P$, Jamieson S, Sinson EB, Beta-1 and beta-2 adrenergic receptor subpopulations im non-failing and fouling human ventricular myocardium: coupling of both receptor subtypes to muscle contraction and relective beta-1 receptor downregulation in heart failure. Circ Res 59, 297-309, 1986.

Brown L, Lorenz B, Erdmann E. Reduced positive inotropic effects in diseased human ventricutar myocirdium. Cardiovasc Res $20,516-520,1986$.

Buttrick $P$, Malhotra A. Factor $S$, Gemen D, Schever J. Effects of chronic dobutamine administration on hearts of normal and hypertensive rats. Circ Res 63, 173-181, 1988.

Chan MY, Dai S, Ko WWW. Effects of morphime on cardiowascular responses to acute myocardial ischemia in rats. Br J Pharmacol 90, 537-543, 1987.

Chatlerjee K, DeMarco $\mathbf{T}$, Rouleaw J-L. Vasodilator therapy in chronic congestive heart failure. Am J Cardiol 62, 46A $-54 \mathrm{~A}, 1988$.

Cleland JGF, Dargie HW, Gillen G, Robertson 1, East BW, Ball SG, Morton JJ, Robertson JIS, Captopril in heart failure: a dowble-blind study of the effects on renal function. I Cardiowasc Pharmacol 8 , 700706,1986

Clozel J-P, Holck M, Osterrieder W, Burkard W, DaPrada M. Effects of chronic myocardial infarction on responsiveness to isopremaline and the state of myocardial beta-adrenoceptors in rats. Cardiovase Res $21,688-695,1987$.

Cody R, Kubo SH, Covit AB, Müler FB. Rutman H, Leonard D, Laragh JH, Feldschuh J, Prelibisz J. Regional blood flow and neurohormonal responses to milrinone in congestive heart failure. Clin Pharmacol Ther 39, 128-135, 1986.

Cody RJ. Renal and hormonal effects of phosphodiesterase III inhibition in congestive heart failure. An J Cardiol 63, 31A-34A, 1989.

Cohn JN, Archibald DG, Ziesche S, Franciosa JA, Harston WE, Tristani FE, Dunkman WB, Jacobs W, Francis GS, Flohr KH, Goldman S, Cobb PR, Shah PM, Saunders R, Fletcher RD, Loeb HS, Hughes WC, Baker B. Effect of vasodilator therapy on mortality in chronic congesitive heart failure. New Engl J Med 314, 1547-1552, 1986.

Cohn JN, Archibald DG, Francis GS, Ziesche $\mathrm{S}$, Franciosa JA, Hartson WE, Tristani FE, Dunkman WB, Jacobs W, Flohr KH, Goldman S, Cobb FR, Shah PM, Sannders R, Fletcher RD, Loeb HS, Hughes VC, Baker B. Veterans administration cooperative study on vasodilator therapy of heart failure: influence of prerandomization variables on the reduction of mortality by treatment wilh bydralazine and isosorbide dinitrate. Circulation 75 (suppl 4), 49-54, 1987.

Cohn JN. Morbidity and mortality. In: Failing Heart Syndrome. Overview of heart failure. 1988, pp 1-11.

Cohn IN. Inotropic therapy for heart failute. New Engl I Med 320, 729-731, 1989

Colucel WS, Wright RF, Braunwald E. New positiwe inotropic agents in the treatment of congestive heart lailure. Now Engl I Mad 314, 290-358, 1986a.

Coluce WS, Wright RF, Bratuwald $\mathbb{E}$. New posituve inotropic agents in the treatment of congestive heart Cailure New Engl I Med 314, 349-358, 1986 b.

Colucei WS. Obserwations on the intracoronary administration of milrinone and dobutamine to patuents will congestive heart fallure. Am J Cardiol 63, 17A-22A, 1989.

Consensus Trial Study Group. Deffects of enalaprill on mortally in severe congestive heart failture. Results of the cooperative North Scandinavian enalapril surviwal sudy (Consensus). New Engl J Med 316 , $1429-1435,1987$.

Cox RH, Bagshaw FJ. Influence of ancsthesia on the response to carotid hypotension in the dog. An $J$ Physiol $237, \mathrm{H} 42,41432,1979$.

Craige E. Heart sounds. In: heart disease. Textbook of cardiovascular medicine (Ed: Braunwald E). WB Sannders, Philadelphia-London-Toronto, 1980 , pp 55-58.

Curtis MJ, Macleod BA, Walker MJA. Models for the study of arrhythmias in myocardial ischemia and infarction: the use of the rat. J Mol Cell Cardiol 19, 399-419, 1987.

Cushman DW, Wang FL, Fung WC, Harvey CM, DeForrest JM. Differentiation of angiotensin conver ting enzyme (ACE) intibitors by their setective inhibition of ACE in physiologically important Larget organs. Am J Hypertens 2, 294-306, 1989. 
Czarnecki CM. Animal models of drug-induced cardionyopathy. Comp Biochem Physiol 796, 9.14, 1984.

Davidson Ir, WR, Banerjee SP, Liang C-S. Dobutanune-induced cardiac adaptationas: comparison with exercise-trained and sedentary rats. Am J Physiol 250, H725-14730, 1986.

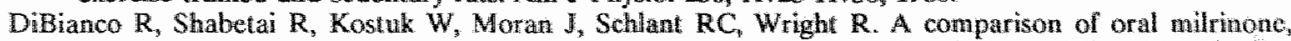
digoxin, and their combination in the treatment of patients with chronic heart faulure. New Engl $\mathrm{J}$ Med 320, 677-683, 1989

Dies F. Intermittent dobutamine in ambulatory patients with chronic cardiac failure. Br I Cha Pract 45 (suppl), 37-40, 1986.

Drexler $H$, Depenbusch $J W$, Truog AG, Zelis R, Flaim SF, Effects of diltuzam on cardiac function and regional blood flow at rest and during exercise in a conscious rat preparation of chronic heart failure (myocardial infarction). Circulation 71, 1262-1270, 1985.

Drexler H, Toggart EJ, Glick MR, Heald J, Flaim SF, Zelis R. Regional vaseular adjustments during recovery from mycoardial unfarction in rats. I Am Coll Cardiol 8, 134-142, $1986 a$.

Drexler H, Flaim SF, Toggart EJ, Glick MR, Zelis R. Cardiocirculatory adjustments to exercise following myocarclial infarction in rats. Basic Res Cardiol 81, 350-360, $1986 \mathrm{~b}$.

Drexler $H$, Depenbusch JW, Truog AG, Zelis R, Flaim SF. Acute regional vascular effects of intravenous captopril in a rat model of myocardial infarction and failure. I Pharmacol Exp Ther 241, 13-19, $1987 \mathrm{a}$.

Drexler $\mathrm{H}$, Faude $\mathrm{F}$, Winterer $\mathrm{H}$, Wollschläger $\mathrm{H}$, Freudenberg $\mathrm{N}$, Just $\mathrm{H}$. Zentrale und regional-vaskuläre hämodynamik von Milrinon bei experimenteller Herzinsuffizienz: Vergleich zu Captopril und Dobutamin. Z Kardiol 76, 507-513, 1987b.

Drexler $H$, Finkh $M$, Honig $S$, Toth $M$, Just $H$, Lang RE. Systenic and regional vascular effects of atrial natrituretic peptide in a rat model of chronic heart failure. Basic Res Cardiol $82,517-529,1987 \mathrm{c}$.

Dunnigan $A$, Staley $N_{A}$, Smith $S A$, Pierpont $M E_{t}$ Judd $D$, Benditt $D G_{n}$ Benson $J_{\mathbb{I}} D W$. Cardiac and skeletal muscle abnormalities in cardiomyopathy: comparison of patients with ventricular tachycardia or congestive heart failure. I Am Coll Cardiol 10,608-618, 1987.

Dzau VJ. Renal and circulatory mechamisms in congestive heart failure. Kidney Int 31, 1402-1415, 1987.

Drau VJ, Re RN. Evidence for the existence of renin in the heart. Circulation 75 (suppl 1), 134-136, 1987.

Earl CQ, Linden J, Weglicki WB. Biochemical mechanisms for the inotropic effect of the cardiotonic drug milrinone. J Cardiovase Pharmacol 8, 864-872, 1986.

Ecker Th, Seitz G. cGMP-kinase concentration in rat heart depends on cardiac vascularization. Naunya Schmiedeberg's Arch Pharmacol 339 (suppl), R70, 1989.

Eichhord EJ, Konstam MA, Weiland DS, Roberts DJ, Martin TT, Stransky NM, Salem DN. Differential effecis of milrinone and dobutamine on right ventricular preload, afterload and systolic perflormance in congestive heart failure secundary to ischemic or idiopathic dilated cardiomyopathy. An I Cardiol $60,1329-1333,1987$.

Eirzig $S$, Staley NA, Metller E, Nicoloff DM, Noren GR. Regimal myocardial blood flow and curdiac function in a naturally occurring congestive cardiomyopathy of turkeys. Cardiovasc Res $14,396-407$. 1980.

Emmert SE, Stabilito II, Sweet CS. Acute and subacute hemodynamic effects of enalaprilat, milrinone and combination therapy in rats with cluronic left ventricular dysfunction. Clin Exp Theor Pract A\%. $297-306,1987$.

Engelmann GL, Vitullo JC, Gerrity RG. Morphometric analysis of cardiac hypertrophy during dcwelopment, maturation, and senescence in spontaneously hypertensive rats. Circ Res $60,487-494,1987$.

Evans DB. Overview of cardiowascular physiologic and pharmacologic aspects of selective phosphodiesterase peak III inhibitors. Am J Cardiol 63, 9A-11A, 1989.

Factor SM, Bhan R, Minase T, Wolinsky H, Sonnenblick EH. Hypertensive-diabetic cardionyopathy in the rat. Am J Pathol 102, 219-228, 1981 .

Farah AE. Historical perspectives on inotropic agents. Circulation 73 (suppl 3 ), 4-9, 1986.

Farah $A E_{*}$ Canniff $P C$, Bentley $R G$, Frangakis $C$. The effect of exiracellutar $\mathrm{Ca} 2+$ and related fons on the cardiac action of milrinone. J Cardiovase Pharmacol 11, 591-600, 11988.

Fein FS, Capasso JM, Aromson RS, Cho S, Nordin C, Miller-Green B, Sonnenblick EH, Factor SM. Combined renovascular hypertension and diabetes in rats: a new proparation of congestive cardiomyopathy. Circulation $70,318-330,1984$ 
Fetdman MD, Copelate L, Gwathmey $\mathbb{~} \mathrm{K}$, Phillips $\mathrm{P}_{\text {, Warten }}$ SE, Schoen FJ, Grossman W, Morgan JP. Deficient production of eyclic AMP: pharmacologic evidence of an important cause of contractile dysfunction in patients with end-stage heart failure. Circulation 75, 331-339, 1987.

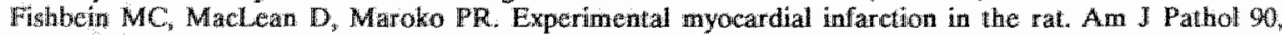
$57.70,1978$.

Fistlabeu $M C$, Lel $L A$, Rubin SA. Long-term propranolol administration alters myocyte and ventricular geometry in rat hearts with and without infarction. Circulation 78, 369-375, 1988.

Flaim SF, Minteder WJ, Nellis SH, Clark DP. Chronic arteriovenous shunt: evaluation of a model for heart fallure in rat. Am I Physiol 236, H698-H704, 1979.

Flaim SF. Peripheral vascular effects of nitroglycerin in a conscious rat model of heart failure. Am Plyysiol 243, H974-H981, 1982.

Forman R, Parmley WW, Sonnenblick EH. Myocardial contractility in relation to hypertrophy and failure in myopathic syrian hamsters. J Mol Cel Cardiol 4, 203-211, 1972 .

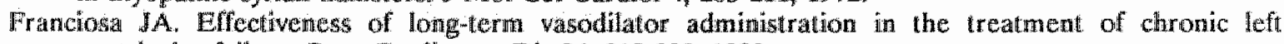
ventricular failure. Prog Cardiovasc Dis 24, 319-330, 1982.

Franciosat JA, wilen M, Ziesche $S$, Cohn JN. Survival in men with sewere chronic left ventricular failure due to either coronary heart disease or idiopathic dilated cardiomyopathy. Am J Cardioll 51, $831-836,1983$.

Francis GS, Goldsmith SR, Levine B, Olivari MT, Cohn JN. The neurohumoral axis in congestive heart failure. An Int Med 101, 370-377, 1984.

Francis GS. Neurohumoral mechanisms involved in congestive heart failure. Am $\mathbf{J}$ Cardiol $55,15 \mathrm{~A}-21 \mathrm{~A}_{\text {, }}$ 1985.

Freis ED. Preload and afterload reduction in treating congestive heart faillure. J Cardiovasc Pharmacol 6 (suppl), 895-902, 1984.

Friberg $\mathbb{P}$, Nordlander M, Lundin S, Folkow B. Effects of ageing on cardiac performance and coronary flow in spontaneously hypertensive and normotensive rats. Acta Physiol Scand 125, 1-11, 1985.

Friberg $\mathrm{P}$, Nordborg C. Functional, morphological and metabolie characteristics of isolated hearts from normotensive and spontaneously thypertensive rats before, during amd after renal hypertension. Acta Physiol Scand 126, 161-171, 1986.

Fylurquist F, Tikkanen I. Atrial natriuretic peptide in congestive heart failure. Am J Cardiol 62, 20A-24A, 1988.

Ganda OP, Rossini AA, Like AA. Studies on streptozotocin diabetes. Diabetes 25, 595-603, 1976.

Ganguly PK, Dhalla KS, Innes IR, Beamish RE, Dhalla NS. Altered norepinephrine turnover and metabolism in diabetic cardiomyopathy. Circ Res 59, 684-693, 1986.

Garrard Jr CL, Weissler AM, Dodge HT. The relationship of alterations in systolic time intervals to cjection fraction in patients with cardiac disease. Circulation 42, 455-462, 1970.

Gcenen DL, Malhotra $A$, Schener J. Regional variation in rat cardiac myosin iso-enzymes and ATPase atclivity after inlarction. Am I" Physiol 256, H745-H750, 1989.

Genesh J. The atrial natriuretic factor. Br Heart If $56,302-316,1986$.

Gert EW. Cardiomyopathic syrian hamster: a possible model of human disease. Prog Exp Tumor Rees $16,242-260,1972$

Gorlin R. Treatment of congestive heart failure: where are we going? Circulation 75 (suppl 4), 108-111, 1987.

Gora L, Marcadier JJ, Schwartz $\mathrm{K}_{n}$ Thorncll LE, Sartore S, Schiaflino $\mathrm{S}$. Myosin types in the human heart. Circ Res 54, 694-702, 1984.

Cuyat GH. The treatment of heart fathure. A methodological review of the literature. Drugs 32, 538-568, 1986.

Gwathmey JK, Hamlin RL. Protection of turkeys against furazolidone-induced cardiomyopathy. Am J Cardiol $52,626-628,1983$

Hälback-Nordlander $M$, Noresson E, Thorén P. Hemodynamic consequences of left ventricular hypertrophy in spontancously hypertensive rats. Am J Cardiol 44, 986-993, 1979.

Handler CE. Emergency medicine: cardiogenic shock. Postgrad Med J 61, 705-712, 1985.

Harris AL, Grant AM, Silver PJ, Evans DB, Alousi AA. Differential wasorelaxant effects of milrinone and amrinone on contractile responses of canine coronary, cerebral, and renal arteries. J Cardiovase Pharmacol $13,238-244,1989$. 
Hayes JS, Bowling N, Pollock GD. Effects of beta-adrenoceptor downregulation on the cardiovascular responses to the stereoisomers of dobutamine. I Pharmacol Exp Ther $235,58-65,1985$.

Herrmann HC, Palacios IF, Dec W, Scheer JM, Fifer MA. Effects of atrial natriuretic factor on coronary hemodynamics and myocandial energetics in patients with heart failure. Am Heart $J 115,1232-1237$, 1988.

Hodgson JMCB, Aja M, Sorkin RP. Intermittent ambulatory dobutamine infusions for patients awating cardiac transplantation. Am J Cardiol 53, 375-376, 1984.

Hodsman GP. Harrison RW, Sumithran E, Johnston CI. Atrial natriuretic peptide and total exchangeablo body sodium: relationships in rats with chronic myocardial infarction. Clin Sci 74, 659-663, 1988a.

Hodsman GP, Kohzuki M, Howes LG, Sumithran $E_{\text {, Tunoda }} \mathbf{K}$, Johnston CI. Neurohumoral responses to chronic myocardial infarction in rats. Circullation 78, 376-381, 1988 b.

Hoffmann $U_{4}$, Axmann $C_{y}$ Palm N. Atrial and ventricular myosins from human hearts. II. Isoenzyme distribution after myocardial infarction. Basic Res. Cardiol 82, 359-369, 1987.

Holmes JR, Kubo SH, Cody RJ, Kligfield P. Milrinone in congestiwe heart failure: observations on ambulatory ventricular arrhythmias. Am Heart J 110, 800 -806, 1985.

Horowits $R$, Winegrad S. cAMP regulation of myosin ATPase activity in the maturing rat hestt. Cire Res $61,914-924,1987$.

Hostetter TH, Pfeffer JM, Pfeffer MA, Dworkin LD, Braunwald E, Brenner BM. Cardiorenal hemodynamics and sodium excretion in rats with myocardial infarction. Am J Physiol 245, H98-1103, 1983.

Inoue M, Kurahashi K, Fujiwara M. Noradrenaline turnower after lefil coronary artery ligation in rat heart. Eur J Pharmacol 143, 251-257, 1987.

Ito $Y$, Suko J, Chidsey CA. Intracellular calcium and myocardial contractility. V. Calcium uptake of sarcoplasmic reticulum fractions in hypertrophied and railing rabbit hearts. J Mol Cell Cardiol 6, 237-247, 1974.

Jalil JE, Doering CW, Janicki JS, Pick R, Shroff SG, Weber KT. Fibrillar collagen and myocardial stiffness in the intact hypertrophied rat left ventricle. Circ Res 64, 1041-1050, 1989.

Jensen RA, Acton EM, Peters. JH. Doxorubicin cardiotoxicity in the rat: comparison of electrocardiogram, transmembrane potential, and structural effects. J Cardiovase Pharmacol 6, 186-200, 1984.

Jensen RA, Acton EM, Peters JH. Doxorubicin cardiotoxicity in the rat: comparison of elcetrocardiogram, transmembrane potential and structural effects (myocardial infarction). Circulation 71 , $1262-1270,1985$.

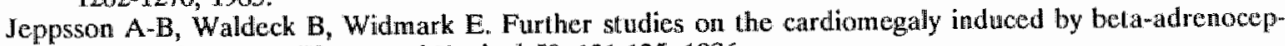
tor agonists. Acta Pharmacol Toxicol 58, 121-125, 1986.

Johnston KM, MacLeod BA, Walker MJA. Responses to ligation of a coronary artery in conscious rats and the actions of antiarrhythmics. Can J Physiol Pharmacol 61, 1340-1353, 1983.

Karam R, Sen S, Wicker P, Healy B. Myosin iso-enzyme post-myocardial infarction. FASEB J 2, A829, 1988.

Karlsberg RP, Penkoske PA, Cryer PE, Corr PB, Roberts R. Rapid activation of the sympathetic nerwots system following coronary artery occlusion: relationship to infarct size and hemodynamic impact. Cardiovasc Res 13, 523-531, 1979.

Katz AM. Potential deleterious effects of inotropic agents in the therapy of chronic heart fatlure. Circulation 73 (suppl 3), 184-190, 1986.

Katz AM. A physiologic approach to the treatment of hearl failume. Hosp Pract 22, $117-148,1987$.

Katz AM. The myocardium in congestive heart failure. Am J Cardiol 63, 12A-16A, 1989.

Kricger EM. Effect of sinoaortic denervation on cardiac oulput. Am J Physiol 213, 1394142, 1967.

Kupper W, Bleifeld W, Hanrath $P$, Mathey D, Effert S. Left ventricular hemodymanics and function un acute myocardial infaretion: stludies during the acute phase, convalescence and late recovery. Am J Cardiol 40, 900-905, 1977.

Kupper W, Erlemeier H-H, Hemm CW. Failure of long-term dobutamine infusion to maintain hemodynamic improvement in patients with sewere heart failure. Circulation $72,405,1985$.

Lakatta EG. Do hypertension and aging thave a similar effect on the myocardium? Circulation 75 (suppl 1), $69-77,1987$.

Laragh $3 H$. Endocrine mechanisms in congestive cardiac failure renin, aldosterone and atrial natriuretic hormone. Drugs 32 (suppl 5), 1-12, 1986. 
Leenen FHH, Chan $\mathrm{YK}^{\mathrm{s}}$, mith $\mathrm{DL}_{\text {, }}$ Reeves RA. Epinephrine and left ventricular function in humans: effects of beta-1 versus non-selective beta-blockade. Clin Pharmacol Ther $43_{n}, 519-528,1988$.

Leier CV, Huss P, Lewiss RP. Drug-induced conditioning in congestive heart failure. Circulation 65 , 13821387,1982 .

Lémonen $H$, Nä̀veri $H$, Mäki $T$, Aittomäki $J_{s}$ Sovijärvi $A$, Kontula $K$, Härkönen M. Reduced lymphocytic beta-adrenergic receptor upregulation in response to exercise in pattients with congestive heart Fuillure. Circulation 76 (suppl 4), 309, 1987.

Lelemtel TH, Maskin CS, Chadwick B, Sonnenblick EH. Clinical response to long-term milrinone therapy in patients with sewere congestiwe heart failure: 12-month experience. In: Milrinone: investigation of wew inotropic therapy for congestive heart failure (eds: Braunwald $\mathrm{E}$ et al). Raven Press, New York, 1984, pp 177-189.

Lejemtel TH, Sonnenblick EH. Should the failing heart be stimulated? New Engl J Med 310, 1384-1385, 1984 ,

LeJemtel TH, Gumbardo D, Chadwick B, Rutman HI, Sonnenblick EH. Milrinone for long-term therapy of severe heart failure: elinical experience with special reference to maximal exercise tolerance. Circulation 73 (suppl 3), 213-218, 1986.

Leflemtel $\mathrm{TH}_{4}$ Scortichini $\mathrm{D}$, Levitt B, Sonnenblick EH. Effects of phosphodiesterase inhibition on skeletal muscle vasculature. Am J Cardiol 63, 27A-30A, 1989.

Lepràn I, Koltai M, Siegmund W, Szekeres L. Coronary artery ligation, early arrhythmias, and determination of the ischemic area in conscious rats. J Pharmacol Meth 9, 219-230, 1983.

Lewine TB. Role of vasodilators in the treatment of congestive heart failure. Am J Cardiol 55, 32A-35A, 1985.

Lewis RP, Rittgers SE, Forester WF, Boudoulas H. A critical review of the systolic time intervals. Circulation $56,146-158,1977$.

Lewis RP, Boudoulas $H_{*}$ Leier CV, Unwerferth DV, Weissler AM. Usefulness of the systolic time intervals in cardiovascular clinical cardiology. Trans Am Clin Climatol Assoc 93, 108-120, 1981.

Liang C-S, Sherman LG, Doherty JU, Wellington K, Lee VW, Hood Jr WB. Sustained improvement of cardiac function in patients with congestive heart failure after short-term infusion of dobutamine. Circulation $69,113-119,1984$.

Lindpaintner K, Jin M, Wilhelm MJ, Suzuki F, Linz W, Schoelkens BA, Ganten D. Intracardiac generation of angiotensin and its ptysiologic role. Circulation 77 (suppl 1), 18-24, 1988.

Lipkin DP, Jones DA, Round JM, Poole-Wilson PA. Abnormalities of skeletall muscle in patients with chronic heart failure. Int \Cardiol 18, 187-195, 1988.

Ludmer PL, Bain DS, Antman EM, Gauthier DF, Rocco MB, Friedman PL, Colucci WS. Effects of millinone on complex wentricular arrhythmias in congestive heart failure secondary to ischemic or idiopathic dilated cardionyopathy. An J Cardiol 59, 1351-1355, 1987.

Maskin CS, Chadwick B, Sonnenblick EH, LeJcmtel TH. Withdrawal and re-institution of long-term milrinone therapy: evidence of drugedependent improvement in cardiac performance without tachyphylaxis. In: Milrinone: investigation of new inotropic therapy for congesilive heart failure (eds: Bratunwald E E al). Raven Pross, Now York, 1984, pp 155-166.

Massic BM, Conway M, Rajagopalan B, Yonge R, Frostick $S$, Ledingham $J_{w}$ Slëght $P$, Rendda G. Sketetal muscle metabolism during cxercise under ischemic conditions in congestive heart failure. Circulation $78,320-326,1988$.

Mauro VF, Mauro LS. Use of intermittent dobutamine infusion in congestive heart failure. Drug Intell Clin Pharm 20, $919-924,1986$.

McKee PA, Castelli WP, McNamara PM, Kannel WB. The matural history of congestive heart failure: the Franingham study. New Engl J Med 285, 1441-1446, 1971.

Meerson FZ. The failing heart: adaptation and deadapuation (ed: Kal2 AM). Rawen Press, New York, 1.983.

Metller FP, Young DM, Ward JM. Adriamycin-induced cardiotoxicity (cardiomyopathy and congestive heart lailure) in rats. Cancer $\operatorname{Res} 37,2705-2713,1977$.

Michel J-B, Lattion A-L, Salzmann J-L, DeLourdes Cerol M, Philippe M, Camilleri J-P, Corvol P. Hormonal and cardiac effects of converting enzyme inhibition in rat myocardial infarction. Circ Res $62,641-650,1988$.

Mirsky I, Pfeffer JM, Pfeffer MA, Braunwaild E. The contractile state as the major determinant in the evolution of left ventricular dysfunction in the spontaneously hypertensive rat. Cire Res 53, 767-778, 1983. 
Monrad ES, Bam DS, Smith HS, Lanoue AS, silveman KI, Gervino EV, Grosisman W. Assessment of long-term therapy with antrinone and the effects of milrinone withdrawal. Circulation 73 (suppl 3 ). $205-212,1986$

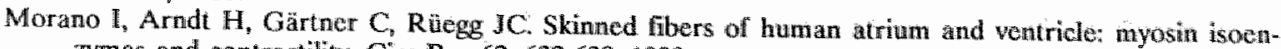
zymes and contractility. Circ Res $62,632-639,1988$.

Morris M, Cain M, Russell A, Ellowt J, Chalmers J. Direct radioimmunoassay of humat plasma atrial vatriuretic peptide in parious normal and pathophysiological stetces: uncrease in tenal and cardiac fallare during exercise. Clin Exp Theor Pract A9, 703-718, 1987.

Nelson GiC, Silke B, Ahuja RC el al. Hemodymamic trial of sequential treatment with dituretic, vasodilator, and positive inotropic drugs in left ventricular failure following acute myocardial infarction. Am Heart J $107,1202-1209,1984$.

Nishinura H, Kubo S, Nishioka A, Imamara $K$, Kawamera K, Hasegawa M. Left ventricular diastolic function of spontaneously hypertensive rats and its relationship to structural conponents of the left ventricle. Clin Sici $69,571-580,1985$.

Nishirmura $H, K u b o S$, Ueyama $M$, Kubota J, Kawamura $K$. Effects of captopril on peripheral hemodyat mics in severe congestive heart failure. Clin Exp Theor Pract A9, 587-591, 1987.

Noma $\mathrm{K}$, Brandle $\mathrm{M}_{*}$ Jacob $\mathrm{R}$. Evaluation of left ventricular function in an axperimental model of congestive hearl failure due to combined pressure and volume overload. Basic Res Cardiol 83 , $58-64,1988$.

Noresson E, Ricksten S-E, Hallbäck-Nondlander $M_{*}$ Thoren P. Performance of the bypertrophied lelt ventricle in spontaneousty hypertensive rats. Effects of changes in preload and aflerioad. Acta Physiol Scand 107, 1-8, 1979a.

Noresson E, Ricksten S-E, Thorén P. Left atrial pressure in normotensive and sponlaneously hypertensive rats. Acta Pbysiol Scand 107, 9-12, 1979b.

Ondett MA, Rubin B, Cushman DW. Desigt of specific inhibitors of angiotensin converting enzyme: a new class of orally active antihypertensive agents. Science $196,44,1977$.

Ondeti MA. Structural relationships of angiotensin converting enzyme inhibitors to pharmacologic activity. Circulation 77 (suppl 1), 74-78, 1988.

Packer M. The role of vasodilator therapy in the treatment of sewere chronic heart lailure. Drugs 32 (suppl 5), 13-26, 1986.

Packer M. Leier CV. Survival in congestive heart fallure during treatment with drugs with positive inotropic actions. Circulation 75 (suppl 4), 55-63, 1987.

Packer M, Lee WH, Kessler PD, Gottlieb SS, Bernstein JL, Kukin ML. Role of neurohormonal nuechanismis in determining survival in patients with severe chronic heart failure. Circulation 75 (suppl 4), $80 \times 2,1987$.

Packer M. Interaction of prostaglandins and angiotersin If in the modultution of renal function in congestive heart fallure. Circulation 77 (suppl 1), $64-73,1988$.

Packer M. Effect of phosphodiesterase inhibitors on survival of patients wh chronic congestive heart. failure. Am J Cardiol 63, 41A-45A, 1989

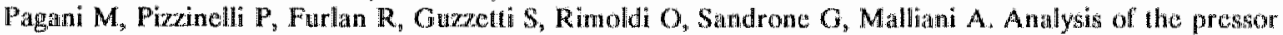
sympathetic rellex produced by intracoronary injections of bradykinin in conscious dogs. Cire Res $56,175-183,1985$.

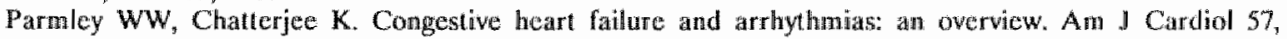
34B-37B, 1986.

Paton DM, Mantel JM. Mechanisms of aclion of the new cardiotonic drugs. Trends Phamacol Sci 9 , $431-432,1988$.

Penpargkul S, Schaible $T$, Yipintsoi $T$, Schewer J. The effect of diabetes on performance and metabolism of rat hearts. Circ Res 47, 911-921, 1980.

Peres AK, Flaim SF, Zelis $R$. Cardiac receptors affect regiomal now during acule infarction in conscious rats. J Auton Nerv Syst 19, 243-254, 1987.

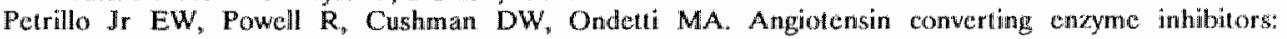
accomplisthments and challenges. Chin Exp Theor Pract A9, 235-241, 1987.

Pfeffer MA, Pfeffer JM, Frohlich ED. Pumping ability of the hypertrophying left ventricle of the spontancously hypertensive rat. Circ Res $38,423,429,1976$.

Pfeffer MA, Pfeffer JM, Fishbein MC, Fletcher PJ, Spadaro J, Kloner RA, Bratunwald E. Myocardial infarct size and ventricular function in rats. Circ Res 44, 503-512, 1979a. 
Pfeffer JM, Pfefter MA, Fishbein MC, Frohlich ED. Cardiac function and morphology with aging in the spontaneoustly hypertensive rat. Am J Physiol 237, H461-H468, 19796.

Pfeffer $J$, Pfeffer $M$, Fletcher $\mathbb{P}$, Braunwald $E$. Alterations of cardiac performance in rats with established spontaneous hypertension. Am J Cardiol 44, 994-998, 1979c.

Pfeffer JM, Pfeffer MA, Mirsky 1, Braunwald E. Regression of left wentricular hypertrophy and prevention of left ventricular dysfunction by captopril in the spontaneously hypertensive rat. Proc Nat Acad Sei USA 79, 3310 3314, 1982a.

Pfeffer JM, Pfeffer MA, Mirsky 1, Steinberg CR, Braunwald E. Progressive ventricular dilatation and dialstolic wall stress in rats with myocardial infarction and failure. Circulation 66 (suppl 2), 264, $1982 \mathrm{~b}$.

Pfeffer JM, Pfeffer MA, Braunwald $E$. Influence of chronic captopril therapy on the infarcted left wentricle of the ratl. Cire Res $57,84-95,1985$ ai.

Pfeffer MA, Pfeffer $J M_{3}$, Steinberg $C$, Finn P. Survival after an experimental myocardial infaretion: beneficial effects of long-term therapy with captopril. Circulation $72,406-412,1985$ b.

Pfeffer JM, Pfeffer MA, Braunwald E... Hemodynamic benefits and prolonged survival with long-term eaptopril therapy in rats with myocardial infarction and heart failure. Circulation 75 (suppl1), $149-155,1987$.

Pfeffer MA, Lamas GA, Vaughan DE, Parisi AF, Braunwald E. Effect of captopril on progressive ventricular dilatation after anterior myocardiat infarction. New Engl J Med 319, 80-86, 1988.

Piscione F, Jaski BE, Wenting Gr, Serruys PW. Effect of a single oral dose of milrinone on left wentricular diastolic performance in the failing human heart. $\mathrm{J}$ Am Coll Cardiol 10, 1294-1302, 1987.

Porta EA, Joun NS, Matsumura L, Nakasone B, Sablan H. Acute adriamycin cardiotoxicity in rats. Res Commun Chem Pathol Pharmacol 41, 125-137, 1983

Ratz P, Ealy R, Flaim SF. Effects of acute myocardial infarction on eardiocirculatory hemodynamics in conscious rat. Fed Proc 39, 633, 1980.

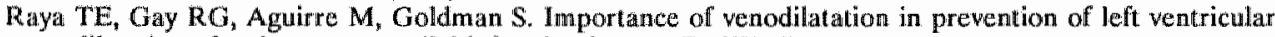
dilatation after large myocardial infarction in rats. FASEB J, 1988a.

Rayat TE, Gay $\mathbb{R G}$, Lancaster L, Aguirre M, Moffett $C$, Goldman S. Serial changes in left ventricular relaxation and chamber stiffness after large myocardial infarction in rats. Circulation $77,1424-1431$, $1988 b$.

Re R. The myocardial intracellular renin-angiotensin system. Am J Cardiol 59, 56,A-58A, 1987.

$\mathrm{Re} \cdot \mathrm{R}$, Rovigatti. $\mathrm{U}$. New approaches to the study of the cellular biology of the cardiovascular system. Circulation 77 (suppl 1), 1.4-18, 1988.

Reiter M. Calcium mobilization and cardiac inotropic mechanisms. Pharmacol Rev 40, 189-217, 1988.

Remme WJ. Congestive heart failure. Pathophysiology and medical treatment. J Cardiovasc Pharmacol 8 (suppl 1), 366.52, 1986.

Rodrigues B, McNeill JH. Comparison of cardiac function in malle and fenale diabetic rats. Gen Pharmacol $18,421-423,1987$.

Roflinan DS et al. Intcrmittent dobutamine hydrochloride infusions in outpatients with chronic congestive uneart failure. Clin Pharm 4, 195-199, 1985.

Risen P, Windeck $P$, Zimmer H-G, Frenzel H, Bürrig KF, Reinauer $H$, Myocardial performance and metabolism in nota-ketotic, diabetic rat hearts" myocardial function and metabolism in vivo and in the isolated perfused heart under the influence of insulin and octanoate. Basic Res Cardiol 81, $620-635,1986$.

Rousson D, Galleyrand J, Silie M, Boissel JP. Uncorrected pre-injection period: a simple non-invasive moasurement for pharmacodynamic screening of inotropic activity. Eur J Pharmacol 31, 559-562, 1987.

Ruffolo Jt RR. Review: the pharmacology of dobutamine. Am J Med Sci 294, 244-248, 1987.

Ruggie N. Congestive heart failure. Med Clin North Am 70, 829-851, 1986.

Rupp $H_{\text {, Wahl }}$, Jacob $R$. Chronic cardiac reactions. IV. Effect of drugs and altered functional loads on carcliac energetics as inferred from myofibrillar ATPase and the myosin isoenzyme population. Basic Res Cardiol 82 (suppl 2), 173-182, 1987.

Schaller MD, Nussberger J, Waeber B, Bussien JP, Turini GA, Brunner H, Brunner HR. Hacmodynamic and pharmacologicall effects of the converting enxyme inhibitor CGS $14824 \mathrm{~A}$ in normal volunteers. Eur J Clin Pharmacol 28, 267-272, 1985.

Schaub $\mathrm{MC}$, Hirzel HO. Alrial and ventricular isomyosin composition in patients with different forms of cardiac hypertrophy. Basic Res Cardiol 82 (suppl 2), 357-368, 1987. 
Schewer J, Buttrick P. The cardiac hypertrophic responses to pathologic and physiologic loads Circulation $75($ suppl 1$), 63-68,1987$.

Schömig A. Adrenergic mechanisms in myocardial infarction: cardiac and systemic çatecholanuine release. J Cardiovasc Pharmacol 12 (suppl1), 1.7, 1988.

Sen $\$$. Regression of cardiac hypertrophy, experimental animal model. Am J Med 26, $87.93,1983$.

Sharma RV, McEldoon JP, Bhalla RC. Age-dependent changes in myosin ATPase activity in the myocardium of spontaneously hypertensive rats. Cardiovasc Res $20,52-60,1986$.

Sharpe $N_{n}$ Murphy J, Smith $H_{n}$ S Hannan. Treatment of patients with symptomless left ventricular dysfunction after myocardial infarction. The Lancet February 6, 255-259, 1988.

Sharpe DN. Angiotensin converting enzyme inhibitors in heart failure: a case for earlier intervention? ACE Rep 59, 1-6, 1989.

Silver PJ. Biochemical aspects of inhibition of cardiovascular low $(\mathrm{Km})$ cyclic adenosine monophosphate phosphodiesterase. Am J Cardiol 63, 2A-8A, 1989.

Simpson PC. Role of proto-oncogenes in myocardial hypertrophy. An J Cardiol 62, 13G-19G, 1988.

Smith TL, Hutchins PM. Central hemodynamies in the developmental stage of spontaneous hypertension in the unanesthetized rat. Hypertension 1, 508-517, 1979.

Simith HJ, Nuttall A. Experimental models of heart failure. Cardiovase Res 19, 181-186, 1985

Smits JFM, Coleman TG, Smith TL, Kasbergen CM, Van Essen H, Struyker-Boudier HAJ. Antihypertensive effect of propranolol in conscious spontaneously hypertensive rats: central hemodynamics, plasma volume, and renal function during beta-blockade with propranolol. I Cardiovase Pharmacol 4, 903-914, 1982.

Smits JFM, Struyker-Boudier HAJ. Systemic and regtonal henodynamics following acute inblition of angiotensin I converting enzyme in the conscious spontaneously hypertensive ral. Prog Pharmacol 5 , $39-51,1984$.

Spodick DH, Doi YL, Bishop RL, Hashimoto T. Systolic time intervalls reconsidered. Re-cwaluation of the pre-cjection period: absence of relation to heart rate. Am J Cardiol 53, 1667-1670, 1984.

Sys SU, Goenen MI, Chalant CH, Brutsaert DL. Inotropic effects of amrinone and millrinone on contraction and relaxation of isolated cardiac muscle. Circulation 73 (suppl 3), 25-35, 1986.

Sys SU, Boels PJ, Brutsaert DL. Positive inotropic and vasodilating effects of amrinone and milrinone in isolated canine heart. J Cardiovasc Pharmacol 10, 445-449, 1987.

Spadaro J, Fishbein MC, Hare C, Pfeffer MA, Maroko PR. Characterization of myocardial infarets in the rat. Arch Pathol Lab Med 104, 179m183, 1980.

Sweet CS, Emmert SE, Stabilito II, Ribeiro LGT. Increased survival in rats with congestive heari failure treated with enalapril. If Cardiovase Pharmacol 10, 636-642, 1987.

Sweet CS, Ludden CT, Stabilito II, Emmert SE, Heyse JF. Beneficial effects of millinone and cnalapril on long-term survival of rats with healed myocardial infarction. Eur J Pharmacoll 147, 29-37, 1988.

Tahiliani $A G, M c N e i l l \mathrm{JH}$. Effects of insulin perfusion and altered glucose concentrations on heart function in 3-day and 6-week diabetic rats. Can J Physiol Pharmacol 64, 1.88-192, 1986.

Takeda $\mathrm{N}$, Ohkubo T, Nakamura I, Suzaki H, Nagano M. Mechanical catecholamine responsiveness and myosin isoenzyme pattern of pressure-overloaded rat ventricular myocardium. Basic Res Cardiol 82 , 370-374, 1987

Thomas RL, Watson D, Marshall LE. Review of intermittent dobutamine infusions ror congestive cardiomyopathy. Pharmacotherapy 7, 47-53, 1987.

Tikkanen T; Svartström-Fraser M, Tikkanen I, Sarioloa H, Fyhrquist F. Hemodynamic effects of auratl natriuretic peptide is rats with heart failure. Eur I Phanmacol 140, 187-193, 1987.

Tomanek RJ, Hovanec JM. The effects of long-term pressure overload and ageing on the myocardium. J Mol Cell Cardiol 13, 471-488, 1981.

Tomanek RJ, Searls JC, Lachenbruch PA. Quantitative changes in the capitlary bed during developing, peak, and stabilized cardiac hypertrophy in the spontancously hypertensive rat. Circ Res 51 , 295-304, 1982 .

Tse J, Powell JR, Baste CA, Pricst RE, Kuo JF. Isoproterenol induced cardiac hypertrophy: nodifications in characteristics of beta-adrenergic receptor, adenylate cyclase, and vemtricular conlraction. Endocrinology 105, 246-255, 1979.

Tsoporis J, Baoxuc Y, Leenen FHH. Arterial vasodilators, cardiac volume load, and cardiac hypertrophy in normotensive rats. Am J Physiol 256, H876-H880, 1989.

Tsunoda K, Mendelsohn FAO, Sexton PM, Chai SY, Hodsman GP, Johnston Cl. Decreased atriat natriuretic peptide binding in renal medulla in rats with chronic heart failure. Circ Res $62,155-161$, 1988. 
Ueda H, Myazaki $T$, Siganuma $Y$, Saito $\mathbb{N}$. heart fallure prone spontaneously hypertensive rats under specific pathogen frec and barricer systems. 1982 pp $155-157$.

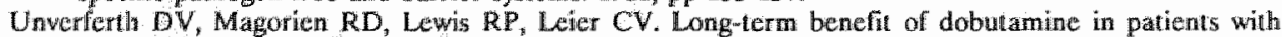
congertive cardionyopathy. An Heart J $100,622-630,1980$.

Unverforh DV, Fertel RH, Thomas i, Leier CV, Croskery R, Hunsaker R, Miller M, Gibb L, Hamlin R. The ewolution of beladadenergic dysfunction during the induction of canine cobalt cardionyopathy. Cardiowalse Res $18,44-50,1984$.

Van det Latarse; A, Bloys vain. Treslong CHF, Vliegen HW, Ricciardi L. Relation between ventricular DNA content and number of myocytes and non-myocytes in hearts of normotensive and sponta neously hypertonsive rats. Cardiowasc Res $21,223-229,1987$.

Van der Wouw PA, Vleeming W, Van Rooij HH, Porsius AJ. Functional response to dobutamine in the pithed tat 48 hours and 7 days after myocardial infarction. Br J Pharmacol 90 (suppl), 20 P, 1987.

Van Gilst WH, De Graeff PA, Wesseling H, De Langen CDJ. Reduction of reperfusion arthythmias in the ischemic isolated rat heart by angiotemsn converting enzyme inhibitors. A comparison of captopril, enalapril and $1 \mathrm{HOE} 498 . \mathfrak{J}$ Cardiovasc Pharmacol 8, 722-728, 1986.

Van Krimpen C, Schoemaker RG, Smits JFM, Daemen MAP, Bosman FT. Elfects of captopril on the cellular response of the myocardium to infarction. Pharmaceut Whbl Sci Ed 10, 235, 1988.

Verrijk R, Vlecming W, Van Rooij HH, Wener J, Porsius. Al. Positiwe inotropic effects of milrinone, whlmazole and AR-L100 on isolated normal and infarcted hegrts of the rat. Arch Int Pharmacodyn Ther 297, 7-17, 1989 .

Vleming W, Van der Wouw PA, Van Rooij HH, Wemer J, Porsius AJ In witro method for measurement of cardiac performance and responses to inotropic drugs after experimentally induced myom cardicil infarction in the rat. I Pharmacol Meth $21,95-102,1989$.

Vogt M, Jacob $R$, Kissling $G$, Rupp H. Chronic cardiac reactions. II. Mechanical and energetic consequences of myocardial tmansformation versus ventricular dilatation in the chromically pressure-loaded heart. Basic Res Cardiol 82 (suppl 2), 147-160, 1987 a.

Vogt M, Jacob R, Noma K, Onegi B, Rupp H. Chronic cardiac reactions. III. Factors involved in the development of structural dilatation. Basic Res Cardioll 82 (suppl 2), 161-172, $1987 \mathrm{~b}$.

Vracko $R$, Thorning $D$, Frederickson $R G$. Connective tissue cells in healing rat myocardium. Am $J$ Pathol 134, $993-1006,1989$.

Waeber $G$, Fasamella d'Amore $T$, Nussberger $J$, Wacber B, Brenner HR. Effect on blood pressure and the renin-angiotensin system of repeated doses of the converting cnzyme inhibitor CGS 14824 A. Eur J Clin Pharmacol 31, 643-646, 3987.

Wallenstcin 5, Zucker COL, Fleiss JL, Some statistical methods useful in circulation rescarch. Circ Res $47,1,9,1980$,

Walsh RA. Cardiovascular effects of the aging process. Am J Med 82 (suppl JB), 34-40, 1987.

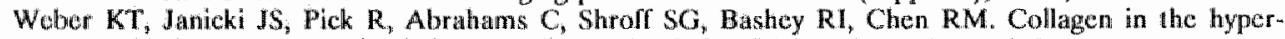
trophicd, pressurcaverloaded myocandiun. Circulation 75 (suppl 1), 40-47, 1987.

Westster AM, Harris WS, Schocmfeld CD. Systolic time intervals in heart failure in man. Circulation 37 , $140.159,1968$.

Weissler AM, Garrard Ir CL. Systolic time intervals in cardiac disease (1). Mod Concepts Cardiovasc Dis $40,1.4,1971$.

Wetzel B, Hatuel N. Now cardiotonic agents. A promising approach for treatment of heart failure. Trends Pharmacol Sci $9,166-170,1988$.

Williams GH. Conwerting onzyne inthibitors in the treatmonl of hypertenion. New Engl $\mathrm{J}$ Med 319 $1517-1525,1988$.

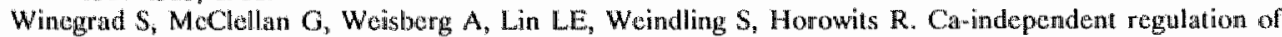
cardiac myosin. Basic Res Cardiol 82 (suppl 2), 183-190, 1987.

Yamori $Y_{,}$Kihara M, Nary Y, Horie R. Myocardial ischemic rals (MIR). Atherosclerosis 42, $15-20,1982$.

Zimmerman MB, Barclay BW, Lehmann M, Norman IA. Effects of chronic administration of angiotensin converting enyyne (ACE) inhibitors on blood pressure and tissue ACE activity in the SHR. Clin Exp Theor Pracl A9, 473-476, 1987.

Zimpler M, Pilt SP, Vatner SF. Elfects of anesthesia on the canine carotid chemoreceptor reflex. Circ $\operatorname{Ros} 48,400-406,1981$. 


\section{SUMMARY}

Although considerable advances have been made in the treatment of congestive heart failure, once the disease is diagnosed, prognosis is still very poor. Therapy is thusfar directed to relieve symptoms rather than to treat the underlying disease. Over the last decade, insight in the cardiac remodeling processes contributing to adaptation and de-adaptation has accumulated. Intervention in the course of these processes may delay progression of the disease and would provide a new opportunity to increase life expectancy. Research in that field implies the availability of a suitable animal model.

The purpose of the present thesis was to develop a rat model to study pathophysiology and treatment of heart failure. The complexity of the syndrome implies the use of intact conscious rats, although studies in anesthetized rats and in in-vitro experiments can contribute to answer specific questions.

The pathophysiology of heart failure has been studied in two possible models, based on the major conditions leading to heart failure in man: chronic hypertension and myocardial infarction (chapters 3 and 4). Because cardiac function is the central problem in heart failure, the cardiac function curve was used as a major determinant of cardiac performance in heart failure. This curve relates cardiac output to cardiac filling pressure. In addition, a broad variety of parameters was measured to obtain more insight in the dynamic interplay of the different mechanisms (chapter 2).

The model of old spontaneously hypertensive rats did not prove to be a practical model, because of selection resulting from a high mortality. In addition, development of high output failure could not be excluded.

Coronary artery ligated rats provided a good model for longitudinal studies on pathophysiology and therapy at different degrees of heart failure. Three stages could be distinguished: a stage of acute heart failure, a stage of hemodynamically compensated heart failure and a stage characterized by a trend to decompensation and increased mortality. In all stages the degree of depression of the cardiac function is related to the size of the infarct.

Effects of therapy have been studied in the latter model, using three types of agents which are known to improve hemodynamics in patients but presumably have different effects on cardiac remodeling. On the one hand, this was performed to evaluate the clinical significance of the model by comparing effects to clinical responses, and on the other hand, to investigate whether different effects on cardiac compensatory mechanisms may result in different hemodynamic effects. Hemodynamic effects of acute and 2 weeks treatment were studied for milrinone, which is not known to affect cardiac remodeling, for dobutamine, which may stimulate cardiac hypertrophy, and for captopril, which may cause regression of cardiac hypertrophy and attenuation of ventricular dilatation (chapters 5,6 and 7). For milrinone, dobutamine and captopril acute effects differed from long-term effects. Although improvements of cardiac performance after therapy from 3-5 weeks after infarction, were comparable for the three drugs, the underlying hemodynamic effects appeared to be different.

Two weeks milrinone treatment restored all hemodynamic changes caused by the infarction at a dose, which does not have acute effects. Intermittent therapy with dobutamine for two weeks resulted in hemodynamic effects comparable to long-term 
milrinone. However, this improvement was measured when dobutamine was not actually circulating, suggesting changes in long-term regulatory mechanisms. A shift in the myocardial myosin iscoenzyme pattern would not only explain the hemodynamic improvement, but also the enhanced inotropic response to acute dobutamine, found after the two weeks intermittent dobutamine therapy.

In contrast to milrinone and dobutamine, captopril therapy from 3 to 5 weeks after infarction restored cardiac performance without an increase in cardiac work. The decreased arterial blood pressure after therapy reduced afterload to the heart. This, however, is not the primary mechanism for the improvement, since after acute captopril treatment and after long-term therapy with a different type of ACE inhibitor, benazepril, the reduction of blood pressure was not accompanied by improved cardiac performance. The possible contribution of different mechanisms has been discussed.

Therapy described above was studied during a rather stable period in the pathophysiology of heart failure. Beneficial effects on cardiac adaptive processes imply an early intervention, i.e. before cell death occurs. Accordingly, effects of earlier captopril treatment were investigated and compared to the effects of the later intervention as described above (chapter 7 ).

No hemodynamic improvement was found after captopril therapy during the first three weeks after infarction. Moreover, although tachycardia and reduced stroke volume preserve cardiac output, these changes indicate deterioration. Results suggest that adaptive processes like cardiac remodeling should take place to a certain extent before captopril exerts beneficial effects on cardiac performance.

From this thesis it can be concluded that in rats with a myocardial infarction, heart failure can be demonstrated by a depressed cardiac function curve. Clinical significance of the model is supported by the observation that improvement of cardiac performance following myocardial infarction in rats is possible with different types of agents which are known to improve hemodynamics in patients. The mechanisms which are responsible for the adaptive processes are the same as the ones which can cause de-adaptation and heart failure. This observation implies that type and timing of therapy for heart failure should be chosen carefully. 


\section{SAMENVATTING}

In de behandeling van hartfalen is de laatste decennia grote vooruitgang geboekt. Desondanks is, als de diagnose hartfalen eenmaal gesteld is, de prognose nog steeds slecht. Tot nu toe lijkt de therapie dan ook meer gericht op het bestrijden van de stuwingssymptomen dan op het behandelen van de onderliggende oorzaak. De laatste jaren is het inzicht in de strukturele veranderingen in het hart, die optreden bij adaptatie- en de-adaptatieprocessen, toegenomen. Interventie in het verloop van deze processen zou het het ziekteproces kunnen vertragen en een nieuwe mogelijkheid bieden de levensverwachting te verhogen. Voor onderzoek op dit gebied is de beschikbaarheid van een goed diermodel van groot belang.

Het doel van dit proefschrift was het ontwikkelen van een goed rattemodel om de pathofysiologie van hartfalen en de effekten van behandeling te bestuderen. Het komplexe karakter van het hartfalen-syndroom impliceert het gebruik van intakte, niet verdoofde ratten, hoewel studies in verdoofde ratten en in-vitro experimenten wel kunnen bijdragen tot het beantwoorden van specifieke vragen.

De pathofysiologie van hartfalen is bestudeerd in twee mogelijke modellen, gebaseerd op de hoofdoorzaken van hartfalen bij mensen: langdurige hypertensie en een hartinfarkt (hoofdstuk 3 en 4). Omdat de hartfunktie het centrale probleem bij hartfalen vormt, is de hartfunktiekurve, die het hartminuutvolume relateert aan de kardiale vuldruk, als belangrijkste maat voor het hartfalen gebruikt. Daarnaast is echter een groot aantal verschillende parameters gemeten om een beter inzicht te verkrijgen in de dynamische interakties van de verschillende mechanismen (hoofdstuk 2).

Het model van oude, spontaan hypertensieve ratten bleek geen praktisch model. De hoge mortaliteit impliceert selektie, terwijl ook een "high output failure" niet kon worden uitgesloten. Ratten met een hartinfarkt vormden een goed model voor longitudinale studies naar de pathofysiologie bij een verschillende mate van hartfalen. Drie stadia konden worden onderscheiden: (1) een stadium van akuut hartfalen, (2) een waarin het verlies aan hartspierweefsel hemodynamisch gekompenseerd is en (3) een stadium waarin een trend naar dekompensatie gevonden wordt en de mortaliteit sterk toeneemt. In alle stadia is de hartfunktie verminderd in direkte relatie tot de grootte van het infarkt.

Effekten van therapie zijn bestudeerd in dit laatste model, waarbij gebruik gemaakt is van drie typen geneesmiddelen. Alle drie geneesmiddelen geven een klinische verbetering in patiënten, terwijl effekten op de struktuur van het hart zouden verschillen. Deze studies zijn enerzijds gedaan om de klinische signifikantie van het model te bepalen door de gevonden resultaten te vergelijken met die bij patiënten, en anderzijds om te onderzoeken of verschillende effekten op de hartstruktuur ook tot verschillen in hemodynamische effekten leiden. De hemodynamische effekten zijn bestudeerd voor milrinon, waarvan niet bekend is dat het strukturele veranderingen in het hart induceert, voor dobutamine waarvan is gevonden dat het kardiale hypertrofie stimuleert en voor captopril, dat een regressie van de kardiale hypertrofie kan veroorzaken en de ventrikeldilatatie kan verminderen (hoofdstuk 5, 6 en 7). 
Voor milrinon, dobutamine en captopril waren de akute effekten verschillend van de lange-termijn effekten. Alle drie gaven een vergelijkbare verbetering van de hartfunktie na twee weken behandelen, terwijl de onderliggende hemodynamische effekten verschillend waren.

Twee weken milrinon-behandeling herstelde alle hemodynamische veranderingen veroorzaakt door het infarkt, bij een dosering die akuut geen effekten zou veroorzaken. Intermitterende dobutamine-therapie gedurende twee weken gaf effekten te zien, vergelijkbaar met die van milrinon-therapie. Deze verbetering werd echter gevonden zonder cirkulerend dobutamine, hetgeen veranderingen in lange-termijn regellsystemen suggereert. Een verschuiving in het kardiale myosine iso-enzympatroon zou niet alleen de verbetering van de hartfunktie verklaren, maar ook dc versterkte inotrope respons op akuut dobutamine na een intermitterende behandeling van twee weken.

In tegenstelling tot milrinon en dobutamine verbetert captopril-behandeling van 3 tot 5 weken na het infarkt de hartfunktie zonder daarbij de arbeid van het hart te verhogen. De verlaagde arteriële bloeddruk verlaagt de nabelasting van het hart, maar lijkt niet het primaire mechanisme voor de hartfunktiewerbetering. Na akute captopril-toediening en na twee weken behandeling met een andere ACE-remmer, benazepril, wordt een vergelijkbare bloeddrukdaling gevonden zonder verbetering van de hartfunktie. Andere mechanismen worden besproken.

De therapie zoals hierboven beschreven is bestudeerd gedurencle een relatief stabiele periode in de ontwikkeling van hartfalen. Voordelige effekten op kardiale adaptatieprocessen impliceren een vroege interventie, dat wil zeggen voordat celdood gaat optreden. Daarom zijn tevens de effekten van vroegere behandeling met captopril onderzocht en vergeleken met de effekten van de latere behandeling (hoofdstuk 7).

Captopril-behandeling gedurende de eerste 3 weken na het infarkt liet geen verbetering van de hartfunktie zien. De tachykardie en gereduceerd slagvolume handhaafden weliswaar het hartminuutvolume, maar gaven eigenlijk een verslechterde toestand weer. De resultaten gaven aan dat kardiale adaptieve processen tot bepaalde hoogte plaatsgevonden moesten hebben voordat captopril-behandeling voordelige effekkten op de hartfunktie heeft.

Uit de resultaten beschreven in dit proefschrift kan gekonkludeerd worden dat in ratten met een hartinfarkt hartfalen aangetoond kan worden door een onderdrukking van de hartfunktiekurve. Verbetering van de hartfunktie in deze ratten is mogelijk met verschillende typen medicijnen, die ook bij patiënten verbetering geven. Dit ondersteunt de klinische relevantie van het model. De observatie dat mechanismen die verantwoordelijk zijn voor de adaptieve processen dezelfde zijn als die welke kunnen leiden tot de-adaptatie en hartfalen maakt dat de keuze van geneesmiddel en van het tijdstip en periode van therapie bij de behandeling van hartfalen zorgvuldig moet gebeuren. 


\section{CURRICULUM VITAE REGIEN SCHOEMAKER}

Naam

14 oktober 1958

1971-1977

$1978-1985$

$1985-1989$

1989

\author{
Regina Geertruida Schoemaker
}

Geboren te Meppel

VWO, Rijksscholengemeenschap, Meppel

Studie biologie aan de Rijksuniversiteit Groningen.

- Hoofdvak Gedragsfysiologie (begeleiding: prof. dr. B. Bohus en dr. J.M. Koolhaas)

- Bijvak Plantenoekologie

- Eerste graads onderwijsbevoegdheid biologie

Wetenschappelijk assistent bij de vakgroep Farmakologie van de Rijksuniversiteit Limburg te Maastricht

Toegevoegd onderzoeker bij de vakgroep Farmakologie van de Rijksuniversiteit Limburg te Maastricht

\section{Publications}

\section{Abstracts}

Fokkema DS, Van de Meulen J, Schoemaker RG, Koolhaas JM. Social stress, blood pressure and respiration in rats. Arch Int Physiol Biochim 91, 24, 1983

Urquhart J, Debets JJ, Schoemaker RG, Struyker Boudier HAJ, Smits JFM. Evaluation of cardiac function in conscious unrestrained rats. Fed Proc 45, 778, 1986

Schoemaker RG, Urquhart JJ, Struyker Boudier HAJ. Effects of dobutamine and amrinone on resting and stimulated cardiac output in conscious unrestrained rats. Naunyn Schmiedeberg's Arch Pharmacol 332 (suppl), R49, 1986

Schoemaker RG, Debets JJ, Smits JFM. Acute hemodynamic effects of myocurdial infarction (MI) in conscious rats. Fed Proc 46, 3022, 1987

Schoemaker RG, Debets JJ, Smits JFM. Coronary artery ligation in conscious rats acate hemodynamic effects. Naunyn Schmiedeberg's Arch Pharmacol 335 (suppl) , 243, 1987

Smits JFM, Schoemaker RG, Debets JJM. Captopril treatment in al conscious rat model for heart failure induced by myocardial infarction. Naunyn Schmiedeberg's Arch Pharmacol 337 (suppl), 274, 1988

Schoemaker RG, Debets JJM, Smits JFM. Cardiac function in a conscious rat model for heart failure after myocardial infarction; effects of captopril treatment. FASEB J 2, 8840, 1988

Van Krimpen C, Schoemaker RG, Smits JFM, Daemen MJAP, Bosman FT. Effects of captopril on the cellular response of the myocardium to infarction. Pharm Wkbl Sci Ed 10, 235, 1988

Schoemaker RG, Debets JJM, Smits JFM. Two weeks milrinone, dobutamine or captopril therapy in a conscious rat model for heart failure induced by myocar- 
dial infarction; hemodynamic effects. Naunyn Schmiedebergs' Arch Pharmacol 339 (suppl), R53 (abstr nr 212), 1989

Schoemaker RG, Debets JJM, Struyker Boudier HAJ, JFM Smits. Effects of 2 weeks dobutamine treatment on heart failure in rats. FASEB Journal 3 (part 1), A437 (abstr nr 1223), 1989

Van Krimpen C, Daemen M, Schoemaker R, Smits J, Bosman F. Effects of captopril (C) on the response of the myocardium to infarction. FASEB Journal 3 (part I), A621 (abstr nr 2294), 1989

Schoemaker RG, Debets JJM, Smits JFM. A conscious rat model for heart failure induced by myocardial infarction; effects of long-term captopril and dobutamine. Br J Pharmacol, in press

\section{Full papers}

Fokkema DS, Koolhaas JM, Van de Meulen J, Schoemaker RG. Social stress induced pressure breathing and consequent blood pressure oscillation. Life Sci $38,569-575,1985$

Schoemaker RG, Urquhart JJ, Debets JJM, Struyker Boudier HAJ, Smits JFM. Acute hemodynamic effects of coronary artery ligation in conscious rats. Basic Res Cardiol, in press

Schoemaker RG, Urquhart JJ, Debets JJM, Struyker Boudier HAJ, Smits JFM. Cardiac function in conscious rats, acute effects of dobutamine and milrinone. Prog Pharmacol, in press

Schoemaker RG, Debets JJM, Struyker Boudier HAJ, Smits JFM. Delayed but not immediate captopril therapy improves cardiac function in conscious rats following myocardial infarction. Submitted for publication

Schoemaker RG, Debets JJM, Struyker Boudier HAJ, Smits JFM. Two weeks intermittent dobutamine therapy restores cardiac performance and inotropic responsiveness in conscious rats with chronic myocardial infarction. Submitted for publication

Schoemaker RG, Debets JJM, Struyker Boudier HAJ, Smits JFM. Beneficial hemodynamic effects of 2 weeks milrinone treatment in conscious rats with heart failure. Submitted for publication 


\section{Ter afsluiting}

Als afsluiting van dit proefschrift wil ik iedereen die op wat voor manier dan ook heeft bijgedragen aan het onderzoek en het tot stand komen van dit werkstuk bedanken.

Allereerst mijn direkte begeleiders in de afgelopen jaren: prof.dr. Harry Struyker Boudier en Dr. Jos Smits. Harry, de verhelderende gesprekken op belangrijke momenten heb ik erg gewaardeerd. De af en toe heftige diskussies hebben bijgedragen aan mijn visie op wetenschappelijk onderzoek. Jos, als mijn direkte begeleider en als persoon heb je me steeds weer gestimuleerd. Je vertrouwen en de vrijheid die je me gegeven hebt zijn voor mij van grote wasrde geweest. De snelle en kritische manier waarop je mijn manuskripten doornam heb ik erg gewaardeerd.

Jacques Debets, jij hebt wat ratten door je handen laten gaan! Zonder jouw bijdrage en inzet was dit onderzoek niet mogelijk geweest. Terwijl jij je vooral met de bio-technische kant van de experimenten bezig hield, stonden tijdens en tussen de metingen Jan Geilen en Jeroen Wilmes altijd klaar om hardware- en software problemen met hun "real-time data aquisition system" op te lossen.

Els Geurts en Mia Hogenboom hebben manuskripten vol strepen en verbeteringen, vergezeld van mondelinge toelichting weten te maken tot dit proefschrift. De kollega's binnen de afdeling hebben gezorgd voor een sfeer waarin het mogelijk was op een plezierige manier te werken.

Tenslotte zou ik de Beoordelingskommissie, Prof.dr. F. Bosman, Dr. H. Drexler, Prof. R. Reneman, Prof. H. Wellens en Prof. P. van Zwieten willen bedanken voor het lezen van het manuskript en de gewaardeerde aanvullingen, terwijl Prof. John Urquhart de puntjes op de i heeft gezet voor wat betreft het Engels . 